\title{
Summary of Federal Construction and Building R\&D in 1994
}

Subcommittee on Construction and Building

Civilian Industrial Technology Committee

National Science and Technology Council

Scott McGaraghan

$Q C$

100

.056

N0.5849

1996 



\section{Summary of Federal Construction and Building R\&D in 1994}

Subcommittee on Construction and Building Civilian Industrial Technology Committee National Science and Technology Council

Scott McGaraghan

September 1996
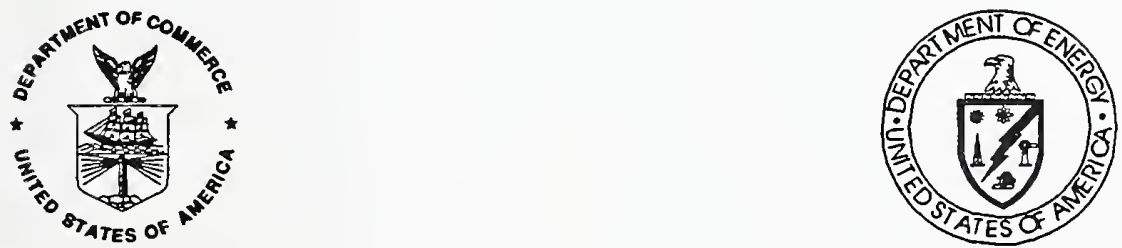

U.S. Department of Commerce

Michael Kantor, Secretary Technology Administration Mary L. Good, Under Secretary for Technology National Institute of Standards and Technology Arati A. Prabhakar, Director
U.S. Department of Energy

Hazel R. O'Leary, Secretary

Energy Efficiency and Renewable Energy Christine A. Ervine, Assistant Secretary 



\section{Table of Contents}

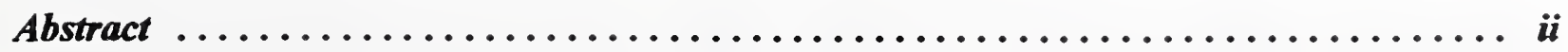

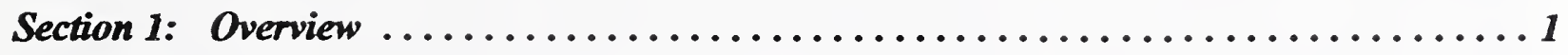

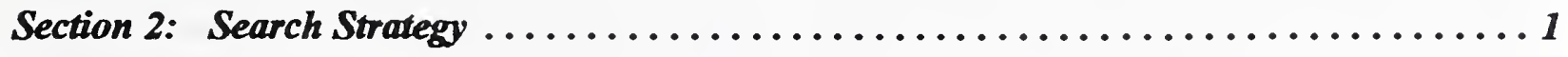

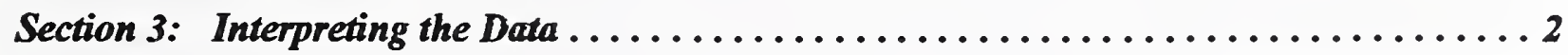

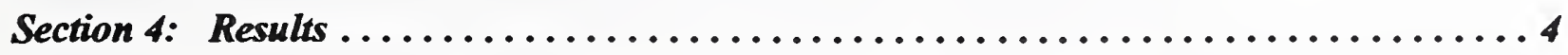

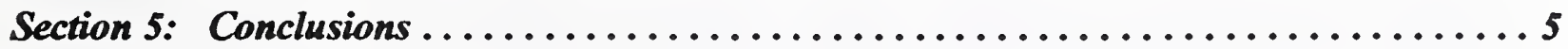

Appendix A: Search Taxonomy $\ldots \ldots \ldots \ldots \ldots \ldots \ldots \ldots \ldots \ldots \ldots \ldots \ldots$

Appendix B: Agency Contacts .............................. 13

Appendix C: Award Spreadsheets.......................... 15

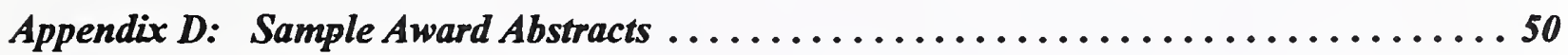





\section{$\underline{\text { Abstract }}$}

In 1994, the federal goverment supported approximately $\$ 70$ billion of resarch and development (R\&D) in many different areas through a wide variety of goverment agencies and contractors. Because the work is spread among so many different entities, it can be difficult to develop a clear picture of the work being done in any one area. In response to this, a new database has ben developed which tracks funding from the appropriation down to the project award level, allowing for comprehensive searches. The National Science and Technology Council's Subcommittee on Construction and Building has studied the database to investigate the status of R\&D in the area of construction and building. The findings of the study are presented herein.

In the course of searching the database, it became apparent that for several reasons the database is not able to provide complete data, largely due to agencies' failure to report vital information. In addition, in-house research performed by some agencies is not defined specifically as $R \& D$, hence does not appear in the database. Despite these omissions, the search results include the vast majority of the work being funded by the federal goverment in the field of construction and building.

The study found that approximately $\$ 566$ million was spent on R\&D for construction and building in 1994. Two agencies, the Department of Defense and the Department of Energy, together account for nearly $75 \%$ of the total. In all, 12 agencies were found to have supported R\&D in this area. Many details on the projects have been included in Appendix $C$ of this report. For additional information, readers should contact individual agencies. Contact numbers are listed in Appendix B. 



\section{Section 1: Overview}

This report describes a search of the Federal R\&D database to identify research and development projects funded by the Federal Government in the field of construction and buildings and the results of that search. The study and a report were requested by the National Science and Technology Council's Subcommittee on Construction and Building. The information contained herein will be used to help coordinate the future Federal R\&D effort in the area of construction and buildings.

This report is comprised of five main sections and four appendices. Section 2 outlines the search process. Due to inconsistency in the reporting practices of the separate agencies, data generated by the search may seem in some places to be incomplete or difficult to understand. Section 3 discusses these inconsistencies and explains how to read the data. The results of the search are presented in Section 4, with conclusions in Section 5. The appendices contain an outline of the search strategy used, a listing of contacts at Federal agencies, and sample listings of project award information found in the search.

\section{Section 2: The Search Process}

The database used for this project, Research and Development in the United States (RaDiUS), is maintained by the Critical Technologies Institute (CTI) of the RAND Corporation in California and consists of two parts: a comprehensive database and a computation and retrieval engine. The data in RaDiUS have been taken directly from information submitted by the individual government agencies which engage in R\&D activities, as defined by the Office of Management and Budget in Circular A-11. The database follows the dollars from Department level appropriations through subordinate agencies, offices, and bureaus, down to the project award level. At the award level, RaDiUS provides project abstracts and detailed information on who was involved, where the project was carried out, when the project was performed, and the project cost. It should be noted that these data are necessarily incomplete where the data provided by the various government agencies and received by CTI are incomplete.

The second element of RaDiUS is a high order, interactive, search, computation and retrieval engine. It allows the user to search by keyword, Federal organization, performer, or fiscal year. All that is required is World Wide Web access using a browser that supports SSL protocol such as Netscape*, and a password furnished by CTI. For this project, a computer terminal at the National Institute of Standards and Technology (NIST) was used to carry out the search. Search results are downloadable using Excel ${ }^{*}$ and a user configurable word processor such as Microsoft Word".

The database contains over 200,000 awards that account for approximately $\$ 70$ billion in R\&D appropriations. Due to the magnitude of RaDiUS, it is necessary to develop a taxonomy of search terms prior to beginning a search such as this. The framework for the taxonomy used for this project

Proprietory software identified here was used for this search. Such identification should not be interpreted as an endorsement of this particular software. 
was the six stages of a building's life: land acquisition, design, construction, operation, renovation, and demolition. Each of these categories was further divided into general issues and specific terms pertaining to each issue. The original version of the taxonomy has been included as Appendix A, although it was continually modified as new search terms were discovered.

It should also be noted at this point that, because RaDiUS is currently in a relatively early stage of development, many of the search strategies must simply be learned by trial and error. Over time, one can become familiar with the vagaries of the system and learn how most effectively to construct searches to minimize "false hits" (those which do not pertain to construction or building) while still ensuring that nothing is missed.

\section{Section 3: Interpreting the Data}

The search results were downloaded from RaDiUS in two separate files: 1) a document with detailed project abstracts by award number (samples of which can be found in Appendix D), and 2) a spreadsheet (Appendix $\mathrm{C}$ ) which includes the following information:

- Project- Title of the project

- Agency- Federal Department or Agency funding the project

- Bureau- subgroup within the Department or Agency

- Program- program within the subgroup

- Award Number- number assigned to the project

- Award Type- indicates type of award

- Start and End Date- dates on which the project starts and ends

- Place of Performance- city and state

- Performer Name

- Perf Type- indicates type of institution/ company performing work

- Perf Parent- Organization of which performer is a part

- Total Award Amount- total amount

appropriated for the entire life of the contract

- Average Annual Funding- Total Award Amount divided by the number of years of the contract (computed by RAND based on either the actual Total Award Amount, or the estimated amount in cases where the actual amount is not given)

- FY Total Amt- expenditures for FY 94 (includes contract awards and agency in-house projects)

- FY Fed Amt- portion of FY 94 expenditures carried by the government

- FY Non-Fed Amt- portion of FY 94 expenditures provided as matching funds by non-government bodies

A note of explanation is required in regard to the funding data. As listed above, three key types of funding information are reported: 1) the Total Award Amount; 2) the Average Annual Funding Amount; and 3) the FY94 Total Funding Amount. Unfortunately, funding information was often incomplete (i.e., some entries were "blank"). These three pieces of information have been provided to allow users to interpret the figures themselves despite inconsistencies among agencies with regard to their spending and reporting practices. 
An example of inconsistency is that Total Award Amount and FY94 Total Amount are not listed in many cases. This results from the reporting practices of the individual agencies. Even if the data is present, it can appear to be inconsistent since, in many cases, a major portion of the funds for a grant or contract are paid to the performer in the first year of the contract (a practice known as "front loading"). The actual amount spent in any given year may not be representative of the average amount spent over the life of the contract. Without contract length information, the Total Award Amount can be misleading as well. Recognizing this, RAND has taken the data given to them and reconstructed the likely average annual funding. This information allows the user to develop a clearer picture of the actual level of funding in a given year.

In most cases, FY94 Total Amount was reported, but Total Award Amount was not. The Department of Agriculture, for example, shows just over \$19 million in FY94 spending, but lists Total Award Amount as zero. Clearly, these two pieces of data are inconsistent. However, the existence of this problem need not call all of the data into question, but serves as a reminder that certain pieces of information have been reported inconsistently.

In short, the most accurate representation of funding levels is found by looking at the Average Annual Funding. Where funding information was provided, the average number will take care of inconsistencies relating to the front loading of contracts. Unfortunately, funding information was sometimes completely blank. As a result, any totals across all projects in the database will underestimate the values for each of the three types of funding information. Of the 1043 awards found in the search, 99, or roughly 10\%, lacked Average Annual Funding data.

In Appendix C, the information has been reproduced in the format listed above. In order to format the spreadsheet so that all the information for a single award could be viewed on two pages, some of the fields have been shrunk, cutting off a portion of the information contained therein. Any questions regarding missing information should be directed to the agencies (contacts are listed in Appendix B).

Because a full printout of project abstracts contained in RaDiUS comprises several hundred pages, they have not been included in this report. However, a sample of the abstracts has been included as an appendix (Appendix D) to provide the reader with a sense of the format in which the abstracts can be downloaded from RaDiUS. Further information can be provided by the agencies.

In some cases a number of awards have identical abstracts. This occurs when a program is funded by a number of different sources. Each of the different funding sources is listed as a separate award, but the same abstract is used. The Award Spreadsheets (Appendix C) also show in some cases that, although the abstracts themselves are identical, the task to which the funds are assigned is different for each different award. An example is the Department of Agriculture's Forest Products Laboratory (CT1060002N0174- CTI060010N0174). The abstracts for these awards are identical, but the supported programs are different. In effect, information on the actual use of the funds comes from the Award Spreadsheets as well as the Award Abstracts. 
There were 1,043 awards found by the search using the taxonomy developed, and some system of classification was needed. In its report, NISTIR 5759, "National Planning for Construction and Building R\&D," the Construction and Building Subcommittee proposed eight products that are needed to achieve the National Construction Goals. Each of the project abstracts was categorized under one of these products areas to provide an idea of the balance of the FY 94 spending. In the spreadsheet, the product is shown in the "Prod. \#" column. The numbers correspond to the products as listed in Table I.

Table 1: Product Areas

\begin{tabular}{|c|l|}
\hline Number & \multicolumn{1}{|c|}{ Product } \\
\hline \hline 1 & Leadership for Innovation \\
\hline 2 & Regulatory Reinvention \\
\hline 3 & Skilled Workforce \\
\hline 4 & Baselines \& Measures \\
\hline 5 & Human Factors \\
\hline 6 & Information Systems \\
\hline 7 & High Perf. Matls. \& Systems \\
\hline 8 & Automation \\
\hline
\end{tabular}

Table II shows the total award amount, average annual funding, and FY 94 total amount for each of the eight product areas (all figures shown are in thousands of dollars). Product 7, "High Performance Materials and Systems proved to be by far the largest category with nearly $\$ 470$ million in Average Annual Funding. This large number is due to the fact that product 7 is defined so as to encompass a wide variety of R\&D not covered by the other categories.

Table II: Funding by Product Number

\begin{tabular}{|l|c|c|c|c|}
\hline \multicolumn{1}{|c|}{ Product } & Number & $\begin{array}{c}\text { Total Award } \\
\text { Amount* }\end{array}$ & $\begin{array}{c}\text { Average Annual } \\
\text { Funding }\end{array}$ & $\begin{array}{c}\text { FY Total } \\
\text { Amt }\end{array}$ \\
\hline Leadership for Innovation & 1 & $\$ 9,961$ & $\$ 2,436$ & $\$ 2,492$ \\
\hline Regulatory Reinvention & 2 & $\$ 1,383$ & $\$ 471$ & $\$ 454$ \\
\hline Skilled Workforce & 3 & $\$ 9,539$ & $\$ 4,128$ & $\$ 3,867$ \\
\hline Baselines \& Measures & 4 & $\$ 3,207$ & $\$ 756$ & $\$ 740$ \\
\hline Human Factors & 5 & $\$ 5,182$ & $\$ 2,438$ & $\$ 1,645$ \\
\hline Information Systems & 6 & $\$ 8,987$ & $\$ 4,070$ & $\$ 2,032$ \\
\hline High Perf. Matls. \& Systems & 7 & $\$ 608,782$ & $\$ 468,998$ & $\$ 325,473$ \\
\hline Automation & $\mathbf{8}$ & $\mathbf{\$ 2 4 , 2 6 3}$ & $\mathbf{\$ 3 3 , 3 8 9}$ & $\mathbf{\$ 2 4 , 6 0 1}$ \\
\hline Total: & & $\$ 671,304$ & $\$ 516,686$ & $\mathbf{\$ 3 6 1 , 3 0 5}$ \\
\hline
\end{tabular}

*All dollar figure shown are in thousands of dollars. 
Table III presents the same information, categorized in this case by Federal Agency. Again, all

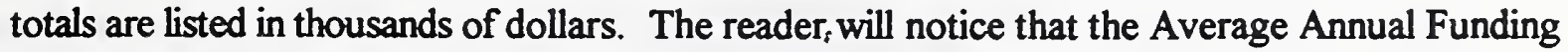
Total listed in Table III exceeds that listed in Table II by $\$ 50$ million. In the process of cross checking the data extracted from RaDiUS with the agencies themselves, it was discovered that there was no funding information listed for the Federal Highway Administration's (FHWA) projects. Apparently, the information sent to RAND included only project abstracts and some other general information, but actual funding figures were excluded. Conversations with FHWA showed that these projects actually represent about $\$ 50$ million in R\&D funding." Accordingly, this figure has been listed at the foot of the table.

Table III: Funding by Federal Agency

\begin{tabular}{|c|c|c|c|}
\hline Department & $\begin{array}{c}\text { Total } \\
\text { Award } \\
\text { Amount }^{\star}\end{array}$ & Average Annual Funding* & FY Total Amt* \\
\hline DOA & $\$ 0$ & $\$ 20,352$ & $\$ 19,189$ \\
\hline DOC & $\$ 0$ & $\$ 16,457$ & $\$ 16,782$ \\
\hline DOD & $\$ 87,897$ & $\$ 292,089$ & $\$ 173,314$ \\
\hline DOE & $\$ 450,880$ & $\$ 122,841$ & $\$ 107,533$ \\
\hline DOI & $\$ 0$ & $\$ 0$ & $\$ 0$ \\
\hline DOJ & $\$ 0$ & $\$ 356$ & $\$ 0$ \\
\hline DVA & $\$ 0$ & $\$ 125$ & $\$ 76$ \\
\hline EPA & $\$ 0$ & $\$ 2,809$ & $\$ 1,097$ \\
\hline HHS & $\$ 0$ & $\$ 11,528$ & $\$ 7,643$ \\
\hline NASA & $\$ 70$ & $\$ 70$ & $\$ 70$ \\
\hline NSF & $\$ 132,456$ & $\$ 50,060$ & $\$ 35,601$ \\
\hline Subtotal: & $\mathbf{\$ 6 7 1 , 3 0 3}$ & $\$ 516,685$ & $\$ 361,305$ \\
\hline \hline DOT & $\$ 0$ & $\$ 50,000 \dagger$ & $\$ 0$ \\
\hline Total: & $\mathbf{\$ 6 7 1 , 3 0 3}$ & $\mathbf{\$ 6 6 6 , 6 8 5}$ & $\$ 361,305$ \\
\hline
\end{tabular}

*All dollar figures shown are in thousands of dollars.

†Figure taken from conversation with Tom Pasko, FHWA.

\section{Section 5: Conclusions}

Due to the problems associated with data reporting described in Sections 2 and 3 above, the information presented in this report must be considered a good estimate rather than a complete and accurate accounting of all Federal R\&D projects. Errors can arise from two basic problems. First, some projects in RaDiUS which pertain to construction and building may not have been found during the search because they did not contain any of the key words used. A more

\footnotetext{
**ersonal communication with Tom Pasko, FHWA, April, 1996.
} 
significant omission comes from the fact that RaDiUS itself may not include complete information for all work being done in the area. Projects which are funded in-house, for example, and which are not funded by specific R\&D appropriations, may not be in the database. In view of these possible exclusions, some cross-checking was done with individual agencies and the funding figures seem to be for the most part in agreement.

Readers interested in more detail on the program areas in which these projects are being performed should refer to Appendix C, where most of the detail is listed. As described above, these spreadsheets provide information necessary to find which agencies are doing work in any given area. For detail on the projects themselves, readers should contact the Agencies through the contacts listed in Appendix B. 


\section{Appendix A: \\ Search Taxonomy}

RaDiUS, the database used in the search process for this report, contains over 200,000 awards which account for approximately $\$ 70$ billion dollars in Federal R\&D funding. A search of a database of this magnitude must be carried out methodically to ensure that all relevant awards are found and to minimize the duplication of effort. Towards this end, a taxonomy of search terms was developed prior to actually begin the search process. This taxonomy, included in the following pages, uses as its basis the six stages of a buildings life:

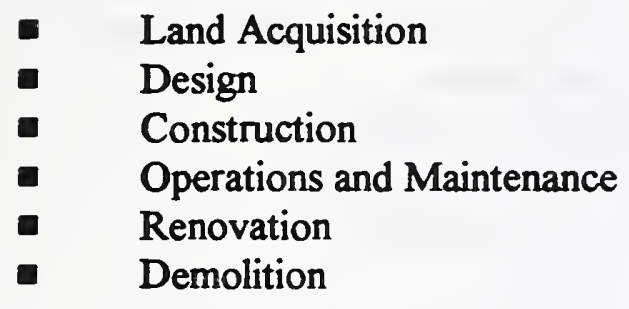

Each of these areas was expanded upon and search terms and phrases relating to each were added. Through the course of the search, new terms were added and existing terms were modified to reflect new understanding of search strategies and possible search areas. 


\section{Taxonomy of Building and Construction Terms}

I. Land Acquisition

A. Industries involved

1. Developers

2. Finance

3. Insurance

B. Issues/Technologies

1. General
a. codes and standards
b. knowledge systems as successors to standards and books
c. integrated project information systems
d. collaborative decision making environments
e. remediation of contaminated construction sites
f. sustainable development (ecological quality)
g. GIS

2. Residential

3. Commercial

4. Industrial

5. Institutional

6. Public Works

C. R\&D Goals

1. Reduce delivery time

2. Reduce project cost

3. Reduce potential for liability from pre-existing problems (contamination, etc.)

II. Design

A. Industries Involved

1. Architectural and engineering design

2. Materials manufacture

B. Issues/Technologies

1. General

a. energy efficiency

i. building orientation, location

ii. materials specification

iii. appliance specification

iv. insulation materials and techniques

v. low emissivity windows (fenestration systems)

vi. building energy utilization monitoring

vii. weatherization

b. automation of design process

i. simulation and visualization

ii. computer aided design 
c. HVAC design

i. indoor air quality

ii. duct work

iii. air changes

iv. air flow rates

v. ozone depletion

vi. CFC replacement

vii. heat pumps

viii. automated HVAC controls

ix. refrigerants

d. structural stability

i. advanced materials

ii. earthquake, flood, hurricane protection (earthquake engineering)

iii. composite materials

iv. experimental stress analysis

v. fracture mechanics

vi. thermal stress analysis

e. lighting

i. adequacy (quality and quantity)

ii. efficiency

iii. daylighting

iv. motion/light sensors

v. lighting controls (automation)

vi. light fixture design

vii. CFLs

viii. electronic ballasts

f. building life expectancy

g. fire protection

i. fire modeling

ii. fire safety

iii. fire dynamics

iv. fire codes

v. flame spread rates

vi. fire suppression

vii. smart fire detection

h. codes and standards

i. safety

ii. energy efficiency

i. integrated data bases and information systems

j. knowledge systems as successors to standards and books

k. integrated project information systems

1. computer-integrated construction

$\mathrm{m}$. whole building systems 
n. certification system

o. human factors

i. cognitive processes and uses of information

ii. physiology

iii. ergonomics

iv. environmental and person-machine interactions

v. team building and work force efficiency

vi. acoustics

2. Residential

p. modeling

a. crime prevention

b. accident prevention

c. [low cost housing]

d. handicap access

3. Commercial

a. handicap access

4. Industrial

a. worker safe design

b. incorporation of environmental equipment and processes

5. Institutional

a. handicap access

6. Public Works

C. R\&D Goals

1. Reduced delivery time

2. Reduced operation and maintenance costs and energy costs

3. Increased productivity and comfort

4. Waste reduction

5. Durability and flexibility of construction

III. Construction

A. Industries Involved

1. Construction

2. Construction equipment manufacture

3. Materials manufacture

4. Installed equipment manufacture

5. Transportation

B. Issues/Technologies

1. General

a. worker safety

i. exposure to toxic materials

ii. exposure to hazardous work conditions

b. speed of construction

i. planning and scheduling (construction management technologies) 
ii. computer aided planning

iii. computer-integrated construction

iv. building technology knowledge base

v. all weather construction

c. durability of construction

i. masonry

ii. shear walls

iii. reinforce concrete

iv. precast concrete

d. excavation techniques

e. robotic construction

i. advanced sensors

ii. building automation systems

f. manufacture of inputs (installed equipment)

g. manufacture of construction equipment

h. high performance materials, components, and systems

i. advanced materials

ii. advanced components

iii. whole building systems

iv. connections

v. assessment and quality assurance technologies

vi. improved water sealants

vii. UV barriers

viii. coatings

i. remediation of contaminated construction sites

j. performance standards for products and processes

2. Residential

3. Commercial

4. Industrial

5. Institutional

6. Public Works

a. underwater construction

C. R\&D Goals

1. Reduced delivery time

2. Reduced operation and maintenance costs and energy costs

3. Waste reduction

4. Durability and flexibility of structures

5. Construction worker safety

IV. Operations and Maintenance

A. Industries Involved

1. Property Management

2. Manufacture of building controls 
3. Maintenance and repair (building and installed equipment)

4. Security

5. Cleaning Services

B. Issues/Technologies

1. General

a. energy efficiency

i. use of controls (lighting, HVAC)

ii. [ease of influencing user behavior]

b. meeting user demands

i. furniture

c. durability [maintaining structures in the most efficient way possible]

d. integrated database and information systems

e. post-occupancy evaluation systems

f. advanced sensors

g. building automation systems

i. building control and management systems

h. functional flexibility

i. indoor air quality

2. Residential

3. Commercial

4. Industrial

a. computer aided facilities management

a. computer aided facilities management

5. Institutional

6. Public Works

a. computer aided facilities management

C. R\&D Goals

1. Reduced O\&M and energy costs

2. Increased productivity and comfort

3. Waste Reduction

V. Renovation

A. Industries Involved

1. Construction

2. Construction equipment manufacture

3. Materials manufacture

4. Installed equipment manufacture

5. Transportation

6. Property Management

B. Issues/Technologies

1. General
a. functional flexibility
b. high performance materials, components, and systems 
I. advanced components

ii. connections

iii. mechanisms, models, and data for life cycle performance

iv. recycling and reuse

c. feasibility of retrofitting old structures with new technologies

d. hazard mitigation (i.e. lead paint, asbestos removal)

e. strengthening and repair of structural members

2. Residential

3. Commercial

4. Industrial

a. planned expansion capacity

5. Institutional

6. Public Works

C. R\&D Goals

a. planned expansion capacity

1. Durability and flexibility of structures

2. Construction worker safety

3. Increased productivity

VI. Demolition
A. Industries Involved
B. Issues/Technologies

1. General
a. post occupancy evaluation systems
b. recycling and reuse
c. worker safety (see Construction)
d. remediation of contaminated sites
e. disposal of building materials
2. Residential
3. Commercial
4. Industrial
5. Institutional
6. Public Works
C. R\&D Goals
1. Waste reduction
2. Worker safety 


\section{Appendix B: \\ Agency Contacts}

The following list is meant to provide the reader with contact information for each of the agencies listed in this report.

Agency

(DOA) Department of Agriculture

(DOC) Department of Commerce

(DOD) Department of Defense

(DOE) Department of Energy

(DOI) Department of Interior

(DOJ) Department of Justice

(DOT) Department of Transportation

\section{Contact}

Hao Tran

Andrew Fowell

Thomas Rutherford

Arthur Rosenfeld

Robert W. Wolf

Cynthia J. Schwimmer

Thomas Pasko
Addreas/Phone

Forest Service, USDA Staff

1 Central Audit

201 14th Street, S.W.

Washington, DC 20250

Attn: HAO TRAN FPHR

(202) 205-1565

National Institute of Standards and

Technology, BFRL

Bldg. 226, Room B250

Gaithersburg, MD 20899

(301) $975-6865$

400 Army Navy Drive

Suite 206

Arlington, VA 22202

(703) 604-5585

Science Advisor, Energy Efficient and Renewable Energy

EE-1, 6A-034

1000 Independence Avenue, S.W.

Washington, DC 20585

(202) 586-6593

Program, Budget \& Liaison Group Bureau of Reclamation

1849 C Street, N.W.

Washington, DC 20240

(202) 208-4690

Financial Management Division Director Office of Justice Programs

Tenth Street and Constitution Ave., N.W.

Washington, DC 20530

(202) $307-3186$

Director, Office of Advanced Research Tumer-Fairbank Highway Research Center 6300 Georgetown Pike

McLean, VA 22101

(703) 285-2679 
(DVA) Department of Veterans Affairs

(EPA) Environmental Protection Agency

Jean Lupinacci

Melvin Myers

(HHS) Department Of Health and Human Services

(NASA) National Aeronautics and Space Administration

Murray Hirschbein

Ken Chong

(NSF) National Science Foundation
Director, Facilities Quality Office (087)

811 Vermont Avenue, N.W.

Washington, DC 20420

(202) $565-4663$

Air Pollution Prevention Division 401 M Street, S.W.

Mail Code 6202J

Washington, DC 20460

(202) 233-9137

Centers for Disease Control and Prevention D-26, 1600 Clifton Road, N.E.

Atlanta, GA 30333

(404) 639-2379

NASA

Code CD

Washington, DC 20546

(202) 358-4262
Director,Structural Systemsand Construction Processes 4201 Wilson Boulevard, Room 545 Arlington, VA 22230

(703) 306-1361 


\section{Appendix $C$ : \\ Award Spreadsheets}

The following pages list all of the projects found in RaDiUS which pertain to construction and building. The format, as described in the text, has been altered slightly from the RaDiUS format to allow for easier viewing. The complete data for any one project can be found by following one row across two facing pages. Each project has been assigned a number (shown in the left-hand column on each page) to allow for easier viewing. Readers who desire further information should consult Appendix B to find contact information for each of the agencies. 


\begin{tabular}{|c|c|c|c|c|c|c|}
\hline & Ageney & Bureau & $\begin{array}{r}\text { Program } \\
\end{array}$ & Project & Award Number & $\begin{array}{l}\text { Award } \\
\text { Type }\end{array}$ \\
\hline 1 & DOA & CSREES & Special research grants & & 91341585868 & $E / G$ \\
\hline 2 & $D O A$ & CSREES & National Research Initiative & Natural resources and environment & 92371037979 & $E / G$ \\
\hline 3 & DOA & CSREES & National Research Initiative & Processing for value added & 92371037986 & $E / G$ \\
\hline 4 & DOA & CSREES & National Research Initiative & Processing for value added & 92371037987 & $E / G$ \\
\hline 5 & $D O A$ & CSREES & National Research Initiative & Processing for value added & 92371038030 & $E / G$ \\
\hline 6 & DOA & CSREES & National Research Initiative & Processing for value added & 92371038031 & $E / G$ \\
\hline 7 & DOA & CSREES & National Research Initiative & Processing for value added & 92371038081 & $E / G$ \\
\hline 8 & $\overline{D O A}$ & CSREES & National Research Initiafive & Processing for value added & 92375008209 & $E / G$ \\
\hline 9 & $D O A$ & CSREES & Special research grants & & 93341588403 & $E / G$ \\
\hline 10 & $D O A$ & CSREES & National Research Initiative & Processing for value added & 93371039268 & $E / G$ \\
\hline 11 & $D O A$ & CSREES & National Research Initiative & Processing for value added & 93371039323 & $E / G$ \\
\hline 12 & $D O A$ & ĊSREES & National Research initiative & Processing fol value added & 93371039395 & $\bar{E} / \bar{G}$ \\
\hline 13 & $\overline{D O A}$ & CSREES & National Research Initiative & Processing for value added & 93371039482 & $E / G$ \\
\hline 14) & $D O \bar{A}$ & CSREES & National Research Initiative & Plant systems & 94371031019 & $E / G$ \\
\hline 15 & $D O A$ & CSREES & National Research Initiative & Markets, trade \& policy & 94371031023 & $E / G$ \\
\hline 16 & $D O A$ & CSREES & McIntire-Stennis cooperative forestry & AL - Alabama (Total \$ from FAADS) & ALA-11-016 & $E / G$ \\
\hline 17 & $\overline{D O A}$ & CSREES & Mclntire-Stennis cooperative forestry & AL - Alabama (Tolal \$ from FAADS) & ALA00974 & $E / G$ \\
\hline 18 & DOA & CSREES & Mclntire-Stennis cooperative forestry & AL - Alabama (Total \$ from FAADS) & ALA00985 & E/G \\
\hline 19 & DOA & CSREES & Payments under the Hatch $\mathrm{AC}$ & $\overline{C A}$ - California (Total \$ from FAADS) & CA-D"-END-4517-H & $\overline{E / G}$ \\
\hline 20 & $D O A$ & CSREES & Payments under the Hatch Act & CA - Califomia (Total \$ from FAADS) & CA-D"-END-5085-H & $E / G$ \\
\hline 21 & $D O A$ & CSREES & Payments under the Hatch $\mathrm{ACt}$ & CA - Califomia (Total \$ from FAADS) & CA-R"-ENT-4315-H & E/G \\
\hline 22 & DOA & CSREES & Payments under the Hatch Act & CO - Colorado (Total \$ from FAADS) & COL00708 & $E / G$ \\
\hline 23 & DOA & FS & Forest protection & Intramural activities & CT1060002N0174 & VIIP \\
\hline 24 & DOA & FS & Resource analyses research & Intramural activities & CTI060004N0174 & VIIP \\
\hline 25 & $D O A$ & FS & Forest management research & Intramural activities & CTI060006N0174 & VIIP \\
\hline 26 & DOA & FS & Forest products and harvesting research & Intramural activities & CT1060010NO174 & VIP \\
\hline 27 & $D O A$ & FS & Ecosystem research & Intramural activities & CT1060012N0174 & VIP \\
\hline 28 & DOA & FS & Forest products and havesting research & Intramural activities & FPL $-4714-01$ & IVIP \\
\hline 291 & DOA & FS & Forest products and harvesting research & Intramural activities & FPL-4714-02 & VIIP \\
\hline 30 & $\overline{D O A}^{-}$ & FS & Forest products and harvesting research & Intramural activities & FPL-4714-03 & VIIP \\
\hline 31 & $\overline{D A}$ & FS & Forest products and harvesting research & Intramural activities & FPL-4714-04 & VIP \\
\hline 32 & $\overline{D O A}$ & FS & Forest products and harvesting research & Intramural activities & IFPL-4715-01 & VIIP \\
\hline 33 & $\overline{D O A}$ & FS & Forest products and harvesting research & Intramural activities & FPL-4715-03 & $\mathrm{VIP}$ \\
\hline 34 & $D O A$ & FS & Forest products and havesting research & Intramural activities & $F P L-4716-02$ & VIP \\
\hline 35 & $\overline{D O A}$ & FS & Forest products and harvesting research & Intramural activities & FPL $-4716-03$ & VIP \\
\hline 36 & $D O A$ & $\overline{F S}$ & Forest products and harvesting research & Intramurai activities & FPL $-4718-02$ & VIIP \\
\hline 37 & $D O A$ & $\overline{F S}$ & Forest products and harvesting research & Intramural activities & FPL-4719-04 & VIP \\
\hline 38 & $D O A$ & CSREES & Payments under the Hatch $A C$ & IL - Illinois (Total \$ from FAADS) & ILU-10-0372 & $E / G$ \\
\hline 39 & $D O A$ & CSREES & McIntire-Stennis cooperative forestry & IN - Indiana (Total \$ from F AADS) & IND059028MS & $E / G$ \\
\hline 40 & DOA & CSREES & Mcintire-Stennis cooperative forestry & KY - Kentucky (Total \$ from FAADS) & KY00642 & $E / G$ \\
\hline 41 & $\overline{D O A}$ & CSREES & Payments under the Hatch $\mathrm{ACt}$ & LA - Louisiana (Total S from FAADS) & LAB02887 & $E / G$ \\
\hline 42 & $D O A$ & CSREES & Mcintire-Slennis cooperative forestry & MN - Minnesota (Total \$ from FAADS) & MIN-43-064 & $E / G$ \\
\hline 43 & $D O A$ & CSREES & Mclntire-Stennis cooperative foresiry & MS - Mississippi (Total \$ from FAADS) & MISZ-6713 & $\overline{E G G}$ \\
\hline 44 & DOA & CSAEES & McIntire-Stennis cooperative forestry & MS - Mississippi (Total \$ from FAADS) & MISZ-6718 & $E / G$ \\
\hline 45 & $D O A$ & CSREES & McIntire-Stennis cooperative forestry & NC - North Carolina (Total \$ from FAADS) & NCZ04141 & $E / G$ \\
\hline 46 & DOA & CSREES & Payments under the Hatch ACt & NY - Now York (Total \$ from FAADS) & NYC-123479 & $E / G$ \\
\hline 47 & $D O A$ & CSREES & McIntire-SIennis cooperative foresiry & PA - Pennsylvania (Total \$ from FAADS) & PEN03088 & $E / G$ \\
\hline 48 & DOA & CSREES & Payments under the Hatch $\mathrm{ACt}$ & PA - Pennsytvania (Total \$ from FAADS) & PEN03343 & E/G \\
\hline 49 & DOA & CSREES & Payments under the Hatch $A$ Ct & SC - South Carolina (Total \$ from FAADS) & SC01476 & $E / G$ \\
\hline 50 & $D O A$ & CSREES & Mcintire-siennis cooperative forestry & SC - South Carolina (Total \$ trom FAADS) & SCZOO194-FR & $E / G$ \\
\hline 51 & $D O A$ & FS & Forest products and harvesting research & Intramural activities & SE-4702-02 & VIP \\
\hline 52 & $D C A$ & FS & Forest protection & Intramural activities & $50-4502-03$ & VIP \\
\hline 53 & $D O A$ & CSREES & Mclntre-Stemis cooperative forestry & TN - Tennessee (Total \$ from FAADS) & TEN00056-MS & $E / G$ \\
\hline 54 & $D O A$ & CSREES & Payments under the Hatch ACt & TX-Texas (Total \$ from FAADS) & TEX08047 & $\bar{E} / \mathrm{G}$ \\
\hline 55 & $D O A$ & CSREES & Payments under the Hatch ACt & VA - Virginia (Total \$ from FAADS) & VA-135443 & $E / G$ \\
\hline 56 & $D O A$ & CSREES & Mcintue-Stennis cooperative forestry & VA - Virginia (Total \$ from FAADS) & VA-136554 & $E / G$ \\
\hline 57 & $D O A$ & CSREES & Payments under the Hatch ACt & WI - Wisconsin (Total \$ from FAADS) & WIS03565 & $E / G$ \\
\hline 58 & $D O A$ & CSREES & Payments unterer the Hatch Act & WI - Wisconsin (Total \$ from FAADS) & WIS03662 & $E / G$ \\
\hline 59 & $\overline{D O A}$ & CSREES & Payments under the Haich ACt & WA - Washington (Total $\$$ from FAADS, & WNP00979 & $E / G$ \\
\hline 60 & DOA & CSREES & McIntire-Stennis cooperative forestry & WA - Washinglon (Total \$ from FAADS) & WNZ-PSE-549 & $E / G$ \\
\hline 61 & $D O C$ & NIST & Building and Fire Research Laboratory & Extramural Activities & 60NANB1675 & $E / G$ \\
\hline 62 & $D O C$ & NIST & Building and Fire Research Laboralory & Extramural Activities & 6ONANB1D 1171 & $E / G$ \\
\hline 63 & $D O C$ & NIST & Building and Fire Research Laboratory & Extramural Activities & 60NANB1D1175 & $E^{\prime} G$ \\
\hline 64 & $D O C$ & NIST & Building and Fire Research Laboratory & Extramural Activities & 6ONANB1D 1176 & $E / G$ \\
\hline 65 & $\overline{D O C}$ & NIST & Building and Fire Research Laboratory & Extramural Activities & 6ONANB1D1177 & $E / G$ \\
\hline
\end{tabular}




\begin{tabular}{|c|c|c|c|c|c|c|c|c|c|c|c|c|c|}
\hline & Agency & $\begin{array}{l}\text { Start } \\
\text { Date }\end{array}$ & $\begin{array}{l}\text { End } \\
\text { Date }\end{array}$ & $\begin{array}{c}\text { Place of } \\
\text { Performance }\end{array}$ & 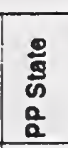 & Performer Name & \begin{tabular}{|c|c|} 
Perf & $=$ \\
\\
Type
\end{tabular} & $\begin{array}{c}\text { Total } \\
\text { Award } \\
\text { Amount }\end{array}$ & $\begin{array}{c}\text { Average } \\
\text { Annual } \\
\text { Fundlng } \\
\end{array}$ & $\begin{array}{c}\text { FY } \\
\text { Total } \\
\text { Amt }\end{array}$ & $\begin{array}{c}\text { FY Fod } \\
\text { Amt }\end{array}$ & \begin{tabular}{|c|} 
FY \\
Non- \\
Fed \\
Amt \\
\end{tabular} & $\begin{array}{c}\text { Prod. } \\
* \\
\end{array}$ \\
\hline & DOA & Mar-91 & Fob-95 & East Lansing & MI & MICHIGAN STATE UNIVER & Edlnst & & 217.663 & & & & 7 \\
\hline & DOA & Sep-92 & Sep-94 & Corvallis & OR & OREGON STATE UNIVERS & Edlnst & & 77 & & & & 7 \\
\hline & DOA & Sep-92 & Sep-94 & Blacksburg & VA & VA POLYTECHNIC INST \& & Edlnst & & 80 & & & & 7 \\
\hline 4 & $D O A$ & Sep-92 & Sep-94 & Madison (Town & WI & UNIVERSITY OF WISCONS & Edlnst & & 118 & & & & 7 \\
\hline & DOA & Sep-92 & Sep-95 & Auburn & $\mathrm{AL}$ & AUBURN UNIVERSITY & Edlnst & & 70 & & & & 7 \\
\hline & DOA & Sep-92 & Sep-95 & Bangor & ME & UNIVERSITY OF MAINE & Edlnst & & 90 & & & & 7 \\
\hline & DOA & Sep-92 & Sep-94 & Mississippi Stat & $M S$ & MISSISSIPPISTATE UNIVE & Edlnst & & 45 & & & & 7 \\
\hline & $D O A$ & Sep-92 & Sep-94 & Cottege Station & $T X$ & TEXAS A\&M RESEARCH F & Edinst & & 76 & & & & 7 \\
\hline & DOA & Jul-93 & Jun-95 & Morgantown & WV & WV UNIVERSITY RESEAR & Edlnst & & 55.996 & & & & 7 \\
\hline 10 & DOA & Sep-93 & Sep-95 & Madison (Town & WI & USDA FOREST SERVICE & Edlnst & & 110 & & & & 7 \\
\hline 11. & DOA & Sep-93 & Sep-95 & Madison (Town & WI & UNIVERSITY OF WISCONS & Edlnst & & 110 & & & & 7 \\
\hline 12 & $D O A$ & Sep-93 & Sep-95 & Moscow & ID & UNIVERSITY OF IDAHO & Edlnst & & 58 & & & & 7 \\
\hline 13 & DOA & Sep-93 & Sep-95 & Corvallis & $O R$ & OREGON STATE UNIVERS & Edlnst & & 55 & & & & 7 \\
\hline 14 & DOA & Sep-94 & Sep-96 & Madison (Town & WI & USDA FOREST SERVICE & Edinst & & 78.928 & 78.928 & 78.928 & 0 & 7 \\
\hline 15 & $D O A$ & Sep-94 & Aug-96 & Pullman & WA & WASHINGTON STATE UNI & Edlnst & & 110 & 110 & 110 & 0 & 7 \\
\hline 16 & $D O A$ & Oct-93 & Sep-98 & Auburn & & SCHOOL OF FORESTRY; $A$ & NorSpec & & & & & & 7 \\
\hline 17 & $D O A$ & Oat-88 & Sep-94 & Auburn & & SCHOOL OF FORESTRY; $A$ & NotSpec & & & & & & 7 \\
\hline 18 & DOA & Oct-90 & Sep-95 & Auburn & & SCHOOL OF FORESTRY; $A$ & NotSpec & & & & & & 7 \\
\hline 19 & DOA & Oct-90 & Sep-95 & Davis & & ENVIRONMENTAL DESIGN & NotSpec & & & & & & 7 \\
\hline 20 & DOA & Oct-93 & Sep-98 & Davis & & ENVIRONMENTAL DESIGN & NorSpec & & & & & & 7 \\
\hline 21 & DOA & Aug-93 & Sep-97 & Riverside & & ENTOMOLOGY; UNIVERSI| & NotSpec & & & & & & 7 \\
\hline 22 & DOA & Jut-94 & Jum-97 & Fort Collins & & CIVIL ENGINEERING: COLI & Nolspec & & & & & & 7 \\
\hline$\overline{23}$ & DOA & Oa-92 & Sep-94 & & & Forest Products Laboratory & NotSpoc & & 3000 & 3000 & 3000 & & 7 \\
\hline 24 & DOA & oct-92 & Sep-94 & & & Forest Products Laboratory & NotSpec & & 1000 & 1000 & 1000 & & 7 \\
\hline 25 & DOA & Oct-92 & Sep-94 & & & Forest Products Laboratory & Noispec & & & & & & 7 \\
\hline 26 & DOA & OC-92 & Sep-94 & & & Forest Products Laboratory & NotSpec & & 15000 & 15000 & 15000 & & 7 \\
\hline 27 & DOA & $O_{c t-92}$ & Sep-94 & & & Forest Products Laboratory & Notspec & & & & & & 7 \\
\hline 28 & DOA & act-91 & Oc-96 & Madison & & FOREST PRODUCTS LABC & FodGov & & & & & & 7 \\
\hline 29 & DOA & Oct-91 & Oct-96 & Madison & & FOREST PRODUCTS LABC & FodGov & & & & & & 7 \\
\hline 30 & DOA & Oct-91 & Oct-96 & Madison & & FOREST PRODUCTS LABC & FodGov & & & & & & 7 \\
\hline 31 & DOA & OCt-91 & Oct-96 & Madison & & FOREST PRODUCTS LABC & FodGor & & & & & & 7 \\
\hline 32 & DOA & Nov-91 & Nov-96 & Madison & & FOREST PRODUCTS LABC & FodGor & & & & & & 7 \\
\hline 33 & $D O A$ & Nov-91 & Nov-96 & Madison & & FOREST PRODUCTS LABC & FedGor & & & & & & 7 \\
\hline 34 & $D O A$ & May-92 & May-97 & Madison & & FOREST PRODUCTS LABC & FodGov & & & & & & 7 \\
\hline 35 & DOA & May-92 & May-97 & Madison & & FOREST PRODUCTS LABC & FodGov & & & & & & 7 \\
\hline 36 & $D O A$ & Nov-90 & Nov-95 & Madison & & FOREST PRODUCTS LABC & FedGov & & & & & & 7 \\
\hline 37 & DOA & Mar-92 & Mar-97 & Madison & & BROWNWOOD FIELD STA & FodGov & & & & & & 7 \\
\hline 38 & DOA & Oct-92 & Sep-97 & Uribana & & AGRI ENGINEERING; UNIV & NotSpec & & & & & & 7 \\
\hline 39 & DOA & Oct-90 & Sep-95 & West Lafayefte & & FORESTRY \& NATURAL RI & NotSpec & & & & & & 7 \\
\hline 40 & DOA & Jul-91 & Jun-96 & Lexington & & FORESTRY; UNIVERSITY d & NotSpeC & & & & & & 7 \\
\hline 41 & DOA & Aug-91 & Dec-93 & Baton Rouge & & AGRI ENGINEERING: LOUI & NotSpec & & & & & & 7 \\
\hline 42 & $\overline{D O A}$ & Oct-89, & Sep-95 & S. Paul & & FOREST PRODUCTS; UNIN & NotSpec & & & & & & 7 \\
\hline 43 & $D O A$ & Jut-91 & Jun-95 & Mississippi Stat & & FOREST PRODUCTS; FOA & NotSpec & & & & & & 7 \\
\hline 44 & DOA & Jul-91 & Jun-95 & Mississippi Stat & & FOREST PRODUCTS; FOA & NotSpec & & & & & & 7 \\
\hline 45 & DOA & Jun-94 & Sep-98 & Raleigh & & WOOD AND PAPER SCIEN & NotSpoc & & & & & & 7 \\
\hline 46 & DOA & Oa-92 & Sep-97 & thaca & & AGRI ENGINEERING; COR & NotSpec & & & & & & 7 \\
\hline 47 & DOA & $\tan -90$ & Dec-94 & University Park & & SCHOOL OF FOREST RES & NotSpoc & & & & & & 7 \\
\hline 48 & DOA & $O_{a t-92}$ & Sep-97 & University Park & & AGRI \& BIOLOGICAL ENGI & Notspec & & & & & & 7 \\
\hline 49 & DOA & Oct-92 & Dec-96 & Clemson & & ENTOMOLOGY: CLEMSON & NotSpoc & & & & & & 7 \\
\hline 50 & DOA & Apr -93 & Jun-95 & Clemson & & FOREST RESOURCES: $\mathrm{CL}$ & NotSpoc & & & & & & 7 \\
\hline 51 & DOA & Dec-88 & DeC-93 & Blacksburg & & SOUTHEASTERN FOREST & FedGov & & & & & & 7 \\
\hline 52 & DOA & Nov-88 & Nov-93 & Gutfport & & SOUTHERN FOREST EXPM & FedGov & & & & & & 7 \\
\hline 53 & DOA & Oa-90 & Sep-95 & Knoxville & & FORESTRY FISHERIES \& & NotSpec & & & & & & 7 \\
\hline 54 & DOA & Apr-91 & Apr-96 & College Station & & AGRI ENGINEERING: TEXA & Notspec & & & & & & 7 \\
\hline 55 & DOA & Jan-94 & Dec-98 & Blacksburg & & AGRI ENGINEERING; VIRG & Notspec & & & & & & 7 \\
\hline 56 & $D O A$ & Jan-91 & Dec-95 & Blacksburg & & WOOD SCIENCE \& FORES & NotSpec & & & & & & 7 \\
\hline 57 & DOA & Oct-92 & Sep-96 & Madisan & & AGRI ENGINEERING; UNIV & NotSpec & & & & & & 7 \\
\hline 58 & DOA & Oct-93. & Sep-97. & Madison & & AGRI ENGINEERING; UNIVI & NotSpec & & & & & & 7 \\
\hline 59 & DOA & JuI-92 & Jun-95 & Puyallup & & ANIMAL SCIENCE: WASHII & NotSpec & & & & & & 7 \\
\hline 60 & DOA & Oct-93 & Sep-95 & Searle & & FOREST RESOURCES; UN & Nolspec & & & & & & 7 \\
\hline 61 & $D O C$ & Sep-94 & Aug-95 & Norwood & $\overline{M A}$ & FACTORY MUTUAL RESEA & NonprofNonEd & & $48.705 i$ & 69.185 & 48.705 & 20.48 & 7 \\
\hline 62 & $D O C$ & Sep-92 & Nov-95 & Piscataway ( $\mathrm{Ne}$ ) & NJ & RUTGERS, THE STATE UN & Edlnst & & 30.191 & & & & 7 \\
\hline 63 & $D O C$ & Sep-92 & Aug-94 & Ann Arbo! & MII & UNIVERSITY OF MICHIGAN & Edlnst & & 68.671 & & & & 7 \\
\hline 64 & $D O C$ & Sep-92 & Aug-94 & Blacksburg & VA & VIRGINIA POLYYTECHNIC II & EdInst & & 60.691 & & & & 7 \\
\hline 65 & $D O C$ & Aug-92 & Aug-94 & Nonwood & MA & FACTORY MUTUAL RESEA & IndBus & & 96.095 & 26 & 26 & & 7 \\
\hline
\end{tabular}




\begin{tabular}{|c|c|c|c|c|c|c|}
\hline & Agency & Bureau & \begin{tabular}{|c} 
Program \\
\end{tabular} & Project & Award Number & $\begin{array}{l}\text { Award } \\
\text { Type }\end{array}$ \\
\hline 66 & DOC & NIST & Building and Fire Research Laboralory & Extramural Activities & 60 NANB2D 1281 & $E / G$ \\
\hline 67 & $D O C$ & NIST & Building and Fire Research Laboratory & Extramural Activities & 6ONANB2D 1290 & $E / G$ \\
\hline 68 & $D O C$ & NIST & Building and Fire Research Laboralory & Extramural Activities & 60NANB2D1291 & $E / G$ \\
\hline 69 & $D O C$ & NIST & Building and Fire Research Laboratory & Extramural Activities & 6ONANB2D1292 & $E / G$ \\
\hline 70 & $D O C$ & NIST & Building and Fire Research Laboratory & Extramural Activities & 6ONANB2D 1300 & $E / G$ \\
\hline 71 & $D O C$ & NIST & Building and Fire Research Laboratory & Extramural Activitios & 6ONANB2H 1232 & $E / G$ \\
\hline 72 & $D O C$ & NIST & Building and Fire Research Laboratory & Extramural Activities & 6ONANB3D1394 & $E / G$ \\
\hline 73 & $D O C$ & NIST & Buriding and Fire Research Laboratory & Extramural Activities & 6ONANB3D1401 & $E / G$ \\
\hline 74 & $D O C$ & NIST & Building and Fire Research Laboratory & Extramural Activities & 6ONANB3D 1409 & $E / G$ \\
\hline 75 & $D O C$ & NIST & Building and Fire Research Laborafory & Extramural Activities & GONANB3D 1413 & $E / G$ \\
\hline 76 & $D O C$ & NIST & Building and Fire Research Laboratory & Extramural Activities & 6ONANB3D 1436 & $E / G$ \\
\hline 77 & $D O C$ & NIST & Building and Fire Research Laboratory & Extramural Activities & GONANB3D 1438 & $E / G$ \\
\hline 78 & $D O C$ & NIST & Building and Fire Research Laboratory & Extramural Activities & 6ONANB3D1439 & $E / G$ \\
\hline 79 & $D O C$ & NIST & Building and Fire Research Laboratory & Extramural Activities & 6ONANB3D1440 & $E / G$ \\
\hline 80 & $D O C$ & NIST & Building and Fire Research Laboratory & Extramural Activities & 6ONANB3D1442 & $E / G$ \\
\hline 81 & $D O C$ & NIST & Building and Fire Research Laboratory & Extramural Activities & GONANB3D1443 & $E / G$ \\
\hline 82 & $D O C$ & NIST & Building and Fire Research Laboratory & Extramural Activities & GONANB3D1444 & $E / G$ \\
\hline 83 & $D O C$ & NIST & Building and Fire Research Laboratory & Extramural Activities & 60NANB4D1531 & $E / G$ \\
\hline 84 & $D O C$ & NIST & Building and Fire Research Laboratory & Extramural Activities & 6ONANB4D 1543 & $E / G$ \\
\hline 85 & $D O C$ & ¿NIST & Building and Fire Research Laboratory & Extramural Activities & GONANB4D 1587 & $E / G$ \\
\hline 86 & $D O C$ & NIST & Building and Fire Research Laboratory & Extramural Activities & 6ONANB4D 1674 & $E / G$ \\
\hline 87 & $D O C$ & NIST & Building and Fire Research Laboratory & Extramural Activities & GONANB4D 1683 & $E / G$ \\
\hline 88 & $D O C$ & NIST & Building and Fire Research Laboratory & Extramural Activities & 6ONANBAD 1705 & $E / G$ \\
\hline 89 & $D O C$ & NIST & Building and Fire Research Laboratory & Extramural Activities & 6ONANB4H1652 & E/CA \\
\hline 90 & $D O C$ & NIST & Building and Fire Research Laboratory & Extramural Activities & 7ONANB1H1173 & $\mathrm{E} / \mathrm{CA}$ \\
\hline 91 & $D O C$ & NIST & Building and Fire Research Laboratory & Extramural Activities & 7ONANB2D1291 & $E / G$ \\
\hline 92 & $D O C$ & NIST & Building and Fire Research Laboratory & Extramural Activities & 7ONANB3D 1300 & $\mathrm{E} / \mathrm{CA}$ \\
\hline 93 & $D O C$ & NIST & Building and Fire Research Laboratory & Extramural Activities & 7ONANB3H 1434 & E/CA \\
\hline 94 & $D O C$ & NIST & Industrial technology services & Advanced Technology Program & 7ONANBAH 1510 & $\mathrm{E} / \mathrm{CA}$ \\
\hline 95 & $D O C$ & NIST & Industrial technology services & Advanced Technołogy Program & 7ONANB4H1517 & E/CA \\
\hline 96 & $D O C$ & NIST & Building and Fire Research Laboratory & Extramural Activities & 70 NANB4H 1554 & E/CA \\
\hline 97 & $D O C$ & NIST & Building and Fire Research Laboratory & STructures Division & CT186103 & VIP \\
\hline 98 & $D O C$ & NIST & Building and Fire Research Laboratory & Structures Division & CTI86106 & VIP \\
\hline 99 & $D O C$ & NIST & Building and Fire Research Laboratory & Building Materials Division & CT186201 & IVIP \\
\hline 100 & $D O C$ & INIST & Building and Fire Research Laboratory & Building Matenals Division & CT186202 & VIP \\
\hline 101 & $D O C$ & NIST & Building and Fire Research Laboratory & Building Environment Division & CT 186301 & VIP \\
\hline 102 & $D O C$ & NIST & Building and Fire Research Laboratory & Building Environment Division & CT186302 & VIP \\
\hline 103 & $D O C$ & NIST & Building and Fire Research Laboratory & Building Environment Division & CT186304 & VIP \\
\hline 104 & $D O C$ & NIST & Building and Fire Research Laboratory & Building Environment Division & CT186305 & VIP \\
\hline 105 & $D O C$ & NIST & Building and Fire Research Laboratory & Building Environment Division & CTI86306 & VIP \\
\hline 106 & $D O C$ & NIST & Building and Fire Research Laboratory & Building Environment Division & CT186307 & VIP \\
\hline 107 & $D O C$ & NIST & Building and Fire Research Laboratory & Building Environment Division & CTI86308 & $\mathrm{I} / \mathrm{IP}$ \\
\hline 108 & $D O C$ & NIST & Building and Fire Research Laboratory & Fire Safety Engineering Division & CT186401 & $\mathrm{I} / \mathrm{IP}$ \\
\hline 109 & $D O C$ & NISTT & Building and Fire Research Laboratory & Fire Safety Engineering Division & CTI86406 & VIIP \\
\hline 110 & $D O C$ & NIST & Building and Fire Research Laboratory & Fire Safety Engineering Division & CT186407 & VIP \\
\hline 111 & $D O C$ & NIST & Building and Fire Research Laboratory & Fire Science Division & CT186501 & I/IP \\
\hline 112 & $D O C$ & NIST & Building and Fire Research Laboratory & Fire Science Division & CT186503 & VIP \\
\hline 113 & $D O C$ & NIST & Building and Fire Research Laboratory & Fire Science Division & CT186504 & VIP \\
\hline 114 & $D O C$ & NOAA & National Ocean Service & Observation \& assessment & NA470R0367 & E/CA \\
\hline 115 & $D O D$ & ARMY & 0602716A - Human Factors Engineering Te & AH70 - Human Factors Engineering Systems D & DAOD4922 & VIIP \\
\hline 116 & $D O D$ & ARMY & 0602784A - Miltary Engineering Technolog & AT 42 - Cold Regions Engineering Technology & DA301642 & VIP \\
\hline 117 & DOD & ARMY & 0601102A - Defense Research Sciences & BH57 - Scientific Problems with Military Applica & DA311314 & $E / G$ \\
\hline 118 & DOD & ARMY & 10601102A - Defense Research Sciences & BH57 - Scientific Problems with Military Applica & DA312039 & $E / C$ \\
\hline 119 & $D O D$ & ARMY & $0601102 A$ - Defense Research Sciences & AT22 - Soil and Rock Mechanics & DA313334 & VIP \\
\hline 120 & DOD & ARMY & $0601102 A$ - Defense Research Sciences & AT22 - Soil and Rock Mechanics & DA313338 & VIP \\
\hline 121 & DOD & ARMY & $0601102 A$ - Defense Research Sciences & AT24 - Snow, Ice and Frozen Soil & DA319229 & VIP \\
\hline 122 & $D O D$ & ARMY & $0601102 A$ - Defense Research Sciences & AT24 - Snow, lce and Frozen Soil & DA319229 & VIP \\
\hline 123 & $D O D$ & ARMY & \$0601102A - Defense Research Sciences & AT24 - Snow, lce and Frozen Soil & DA319230 & VIIP \\
\hline 124 & $D O D$ & $A R M Y$ & $0603102 A$ - Materials \& Siruciure Advance & DDO1-COMBAT ENGINEERING COMPONEN & DA319715 & TIP \\
\hline 125 & $D O D$ & ARMY & $0602784 \mathrm{~A}$ - Military Engineering Technolog & AT40 - Mobility \& Weapons Effects Tectinology & DA320002 & IVIP \\
\hline 126 & $D O D$ & ARMY & 0602784 A - Military Engineering Technolog & AT40 - Mobility \& Weapons Effects Technology & DA320004 & IVIP \\
\hline 127 & $D O D$ & ARMY & $0601102 A$ - Defense Research Sciences & AT22 - Soil and Rock Mechanics & DA320020 & V/P \\
\hline 128 & $D O D$ & ARMY & 0602784 A - Military Engineering Technolog & AT40 - Mobility \& Weapons Effects Technology & DA320029 & VIP \\
\hline 129 & $D O D$ & ARMY & $0601102 A$ - Defense Research Sciences & AT22-Soil and Rock Mechanics & DA320068 & VIP \\
\hline 130 & $D O D$ & ARMY & 10602784A - Military Engineering Tochnolog & AT40 - Mobility \& Weapons Effects Technology & DA320070 & VIP \\
\hline
\end{tabular}




\begin{tabular}{|c|c|c|c|c|c|c|c|c|c|c|c|c|c|}
\hline & Agency & $\begin{array}{l}\text { Start } \\
\text { Date }\end{array}$ & $\begin{array}{l}\text { End } \\
\text { Date }\end{array}$ & $\begin{array}{c}\text { Place of } \\
\text { Performance }\end{array}$ & 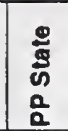 & Performer Name & $\begin{array}{l}\text { Pert } \\
\text { Typo }\end{array}$ & $\begin{array}{l}\text { Total } \\
\text { Award } \\
\text { Amount }\end{array}$ & $\begin{array}{l}\text { Average } \\
\text { Annual } \\
\text { Fundlng }\end{array}$ & $\begin{array}{c}\text { FY } \\
\text { Total } \\
\text { Amt }\end{array}$ & $\begin{array}{c}\text { FY Fed } \\
\text { Amt }\end{array}$ & \begin{tabular}{|c|} 
FY \\
Non- \\
Fod \\
Amt \\
\end{tabular} & Prod. \\
\hline 66 & $D O C$ & Aug-92 & May-95 & Clemson & sc & CLEMSON UNIVERSITY & Edlnst & & \begin{tabular}{|l|}
38.204 \\
\end{tabular} & 53.879 & \begin{tabular}{|l|}
53.879 \\
\end{tabular} & 0 & 7 \\
\hline 67 & $D O C$ & Aug-92 & Sep-95 & Pullman & WA & WASHINGTON STATE UNI & Edlnst & & 69.947 & 70.465 & $70.465 \mid$ & 0 & 7 \\
\hline 68 & $D O C$ & Sep-92 & Aug-94 & West Lafayette & IN & PURDUE RESEARCH FOU & EdInst & & 79.381 & & & & 7 \\
\hline 69 & $D \infty$ & Aug-92 & Aug-94 & Washington & $\overline{D C}$ & GEORGE WASHINGTONL & PriEdlnst & & 94.707 & & & & 7 \\
\hline 70 & $D O C$ & Sop-92 & Aug-94 & College Park & MD & UNINERSITY OF MARYLAI & NEdlnst & & 50.886 & 7.056 & 7.056 & 의 & 7 \\
\hline 71 & $D O C$ & May-94 & Apr-95 & Ann Arbor & MI & MODERNIZATION FORUM & NonproinonEc & & 275 & 275 & 275 & 0 & 7 \\
\hline 72 & $D O C$ & Fob-93 & Feb-94 & University Park & PA & PENNSYLVANIA STATE U & EdInst & & 58 & & & & 7 \\
\hline 73 & $D O C$ & Apr-93 & $D_{90-93}$ & Ann Arbor & MI & UNIVERSITY OF MICHIGA & Edlnst & & 58.543 & & & & 7 \\
\hline 74 & $D O C$ & May-93 & Apr-94 & Now York & NY & COLUMBIA UNIVERSITY & PriEdlnst & & 200 & & & & 7 \\
\hline 75 & $D O C$ & May-93 & Doc-93 & Boston & MA & NEW ENGLAND AOUARIU & NonproNonEC & & 200 & & & & 7 \\
\hline 76 & $D O C$ & Sep-93 & Sep-94 & Austin & TX & UNIVERSITY OF TEXAS & Edinst & & 44.634 & & & & 7 \\
\hline$\pi$ & $D O C$ & Sep-93 & Aug -95 & Berkeley & $C A$ & REGENTS OF UNIVERSIT & Edinst & & 83.834 & 99.667 & 99.667 & 0 & 7 \\
\hline 78 & $D O C$ & Sep-93 & Aug-95 & Cambridge & MA & MASSACHUSETTS INSTIT & PriEdlnst & & 81.012 & 86.522 & 86.522 & 0 & 7 \\
\hline 79 & $D O C$ & Sep-93 & Sep-95 & Asn Arbor & MI & MICHIGAN UNIVERSITY & Edlnst & & 43.348 & 53.721 & 46.695 & 7.026 & 7 \\
\hline 80 & $D O C$ & Sep-93 & Aug-94 & Chicago & IL & UNIVERSITY OF ILLINOIS & Edlnst & & 99.82 & & & & 7 \\
\hline 81 & $D O C$ & Sep-93 & Sep-94 & Lexingion & KY & UNIVERSITY OF KENTUCF & KriEd/KENTU & CKY UN & 48.008 & & & & 7 \\
\hline 82 & $D O C$ & Sep-93 & Oct-95 & Pasadena & $\overline{C A}$ & CALIFORNIA INSTITUTE O & NonprofiNonEc & & 59.258 & & & & 7 \\
\hline 83 & $D O C$ & Dec-93 & Nov-94 & Columbia & $M D$ & HUGHES ASSOCIATES & IndBus & & 99.503 & 111.5 & 99.503 & 12 & 8 \\
\hline 84 & $D O C$ & Sep-94 & Sep-95 & Bozeman & MT & MONTANA UNIVERSITY & Edlnst. & & 200 & 278 & 200 & 78 & 7 \\
\hline 85 & $D O C$ & Jun-94 & Nov-95 & ST. Louis (Coun & MO & WASHINGTON UNIVERSIT & TriEdlnst & & 20.992 & 20.992 & 20.992 & 0 & 7 \\
\hline 86 & $D O C$ & Sep-94 & Aug-95 & Lexinglan & KY & KENTUCKY RESEARCH FC & CEdinst & & 30.302 & 30.302 & 30.302 & 0 & 7 \\
\hline 87 & $D O C$ & Sep-94 & Aug-95 & Dearbom & MI & TOMES VAN RICKLEY \& A & OOTh & & 24.977 & 24.977 & 24.977 & 0 & 7 \\
\hline 88 & $D O C$ & Sep-94 & Sep-95 & & $D C$ & NATIONAL INSTITUTE OF & INonprofNonEc & & 24.996 & 59.996 & 24.996 & 35 & 7 \\
\hline 89 & $D O C$ & Sep-94 & Aug-95 & Princeton & $M D$ & KANSAS STATE UNIVEAS & Edlnst & & 38.206 & 47.775 & 38.206 & 9.569 & 7 \\
\hline 90 & $D O C$ & Sep-92 & Aug-95 & College Park & MD & UNIVERSITY OF MARYLAR & Edinst & & 65.927 & 64.577 & 64.577 & 0 & 7 \\
\hline 91 & $D O C$ & Sep-94 & Aug-95 & West Lafayette & IN & PURDUE RESEARCH FOU & EdInst & & 73.121 & 78.121 & 73.121 & 5 & 7 \\
\hline 92 & $D O C$ & Sep-94 & Aug-95 & College Park & NOD & MARYLAND UNIVERSITY & PriEdinst & & 75.41 & 79.85 & 75.41 & 4.44 & 7 \\
\hline 93 & $D O C$ & Sep-93 & Sep-95 & Biacksburg & VA & VIRGINIA POLYTECHNICI & Edilnst & & 60 & 60 & 60 & 0 & 7 \\
\hline 94 & $D O C$ & Feb-94 & Feb-96 & Briarcliff Manor & NY & PHILIP'S LABORATORIES & IndBus & & 667 & 898 & 667 & 231 & 7 \\
\hline 95 & $D O C$ & Feb-94 & Mar-95 & Wayne & NJ & THOMAS ELECTRONICS & IndBus & & 243.1 & 243.1 & 243.1 & 0 & 7 \\
\hline 96 & $D O C$ & Sep-94 & Feb-96 & Santa Monica & CA & INSTITUTE FOR RESEARC & IndBus & & 188 & 423 & 188 & 235 & 7 \\
\hline 97 & $D O C$ & Oct-92 & Sep-94 & & & Structures Division & NotSpec & & 595.25 & 523.1 & 523.1 & & 7 \\
\hline 98 & $D O C$ & Oct-92 & Sep-94 & & & Structures Division & NotSpec & & 1487.85 & 1383.4 & 1383.4 & & 7 \\
\hline 99 & $D O C$ & Oct-92 & Sep-94 & & & Building Materials Division & NotSpec & & 429.15 & 454.9 & 454.9 & & 7 \\
\hline 100 & $D O C$ & Oct.92 & Sep-94 & & & Building Materials Division & NotSpec & & 634.3 & 786.5 & 786.5 & & 7 \\
\hline 101 & DOC & Oct-92 & Sep-94 & & & Building Environment Divisio & NotSpec & & 708 & $758 . \overline{9}$ & 758.9 & & 7 \\
\hline 102 & $D O C$ & Oct-92 & Sep-94 & & & Buritding Environment Divisio & NotSpec & & 389.95 & 390.3 & 390.3 & & 7 \\
\hline 103 & $D O C$ & Oa-92 & Sep-94 & & & Building Environment Divisio & Noispec & & 419.45 & 439.6 & 439.6 & & 7 \\
\hline 104 & $D O C$ & Oct-92 & Sep-94 & & & Building Emvironment Divisio & NotSpec & & 792.8 & 1194.9 & \begin{tabular}{|l|}
1194.9 \\
\end{tabular} & & 8 \\
\hline 105 & DOC & Oct-92 & Sep-94 & & & Building Environment Divisio & NotSpec & & 299.85 & 300.1 & 300.1 & & 7 \\
\hline 106 & $D O C$ & Oct-92 & Sep-94 & & & Building Environment Divisio & Noispec & & 99.7 & 99.6 & 99.6 & & 7 \\
\hline 107 & $D O C$ & Oct-92 & Sep-94 & & & Building Environment Divisio & Notspec & & 740.6 & 991.3 & 991.3 & & 7 \\
\hline 108 & $D O C$ & $O_{c 1-92}$ & Sep-94 & & & Fire Safety Engineering Divis & NotSpec & & 781.3 & 580.2 & 580.2 & & 7 \\
\hline 109 & $\bar{D} c$ & Oct-92 & Sep- 94 & & & Fire Safety Engineering Divis & NotSpec & & 1208.35 & 1025.7 & $10 \overline{25} .7$ & & 7 \\
\hline 110 & $D O C$ & Oct-92 & Sep-94 & & & Fire Safety Engineering Divis & SNotSpec & & 1331.65 & 1496.7 & 1496.7 & & 7 \\
\hline 111 & $D O C$ & Oct-92 & Sep-94 & & & Fire Science Division & NotSpec & & 653.65 & 667.3 & 667.3 & & 7 \\
\hline 112 & $D O C$ & Oct.92 & Sep-94 & & & Fire Science Division & NotSpec & & 756.9 & 800.8 & 800.8 & & 7 \\
\hline 113 & $D O C$ & Oct-92 & Sep-94 & & & Fire Science Division & NotSpec & & 1128.15 & 1298 & 1298 & & 7 \\
\hline 114 & $D$ & Sep-94 & Aug-95 & Boston & MA & COMMONWEALTH OF MA & ¿StaLOCGov & & 300 & 428.57 & 300 & \begin{tabular}{|l|}
128.6 \\
\end{tabular} & 7 \\
\hline 115 & $D O D$ & Jul-87 & & & & NO PERFORMER INFO AV & NotSpec & & 365 & & & & 5 \\
\hline 116 & $D O D$ & aci-84 & Sep-94 & & & NO PERFORMER INFO AV & NotSpec & & 210 & 212 & 212 & & 7 \\
\hline 117 & DOD & $0 c t-86$ & Jun-94 & Cambridge & & MASSACHUSETTS INSTIT & PriEdIMASS, & 1948 & 243.5 & & & & 7 \\
\hline 118 & $D O D$ & Jan-87 & Jun-94 & Cambridge & & MASSACHUSETTS INSTIT & PriEdIMASS & 6877 & 859.625 & & & & 8 \\
\hline 119 & DOD & OCt-88 & Sep-01 & & & NO PERFORMER INFO AV & Norspec & & 218.667 & 201. & 201 & & 7 \\
\hline 120 & DOO & $0 a-87$ & Sep-94 & & & NO PERFORMER INFO AV & Noctspec & & 174 & 177 & 177 & & 7 \\
\hline 121 & DOD & $00-89$ & Sep-99 & & & NO PERFORMER INFO AV & Notspec & & 114 & 152 & 152 & & 7 \\
\hline 122 & 000 & $0 a-89$ & Sep-99 & & & NO PERFOORMER INFO AV & NotSpec & & 114 & 152 & 152 & & 7 \\
\hline 123 & $D O D$ & Oct-91 & Sep-99 & & & NO PERFORMER INFO AV & Notspec & & 143 & 151 & 151 & & 7 \\
\hline 124 & $D O D$ & Oct-91 & Jan-95 & & & NO PERFORMER INFO AV & & & 154.5 & & & & 7 \\
\hline 125 & $\overline{D O D}$ & $0 a-89$ & Sep-99 & & & NO PERFORMER INFO AV & NotSpec & & 252 & 254 & 254 & & 8 \\
\hline 126 & $D O D$ & Oct-89 & Sep-97 & & & N NO PERFORMER INFO AV & NotSpec & & 222 & 231 & 231 & & 7 \\
\hline 127 & DOD & Oct-90 & Sep-94 & & & NO PERFORMER INFO AV & NotSpec & & 178 & 184 & 184 & & 7 \\
\hline 128 & $D O D$ & $00-86$ & Sep-94 & & & NO PERFORMER INFO AV & NotSpec & & 318 & 100 & 100 & & 7 \\
\hline 129 & DOD & OCA-91: & Sep-97 & & & INO PERFORMER INFO AV & No!Spec & & 288.333 & 329 & 329 & & \\
\hline 130 & DOD & Oct-91 & Sep-01 & & & INO PERFORMER INFO AV & NotSpec & & 129 & 108 & 108 & & 7 \\
\hline
\end{tabular}




\begin{tabular}{|c|c|c|c|c|c|c|}
\hline & Agency & Bureau & $\begin{array}{c}\text { Program } \\
\end{array}$ & Project & Award Number & $\begin{array}{l}\text { Award } \\
\text { Type }\end{array}$ \\
\hline 131 & DOD & ARMY & 0602720 A - Environmental Quality Technok & AF25 - Miliary Environmental Restoration Techr & DA320090 & VIP \\
\hline 132 & $D O D$ & ARMY & 0602784 A - Military Engineering Technolog & AT40 - Mobility \& Weapons Effects Technology & DA320105 & VIP \\
\hline 133 & $D O D$ & ARMY & $0601102 A$ - Defense Research Sciences & AT22 - Soll and Rock Mochanics & DA320110 & VIP \\
\hline 134 & $D O D$ & ARMY & 0602211 A - Aviation Technology & A47A - Aeronautical \& Aircraf Weapons Techne & DA320952 & $E / C$ \\
\hline 135 & DOD & ARMY & 0605810 A - RDT\&E Support for Nondevelo & DE65 - NDI Testing & DA325493 & UIP \\
\hline 136 & $D O D$ & ARMY & 0601102A - Defense Research Sciences & BH57 - Scientific Problems with Military Applica & DA328912 & $E / C$ \\
\hline 137 & $D O D$ & ARMY & 0603308 A - Army Missile Defense Sys Inted & Project number unspocified & DA337099 & E/C \\
\hline 138 & $D O D$ & ARMY & 0603308 A - Army Missile Defense Sys Inted & Project number unspecified & DA347123 & E/C \\
\hline 139 & DOD & ARMY & 0601 102A - Defense Research Sciences & BH57 - Scientific Problems with Military Applica & DA351026 & E/C \\
\hline 140 & $D O D$ & ARMY & $0602784 \mathrm{~A}-$ Military Engineerning Technolog: & AT 40 - Mobility \& Weapons Effects Technology & DA359209 & UIP \\
\hline 141 & $D O D$ & ARMY & 0602784A - Matitary Engineering Technolog & AT40 - Mobility \& Weapons Effects Tochnology & DA359210 & VIP \\
\hline 142 & $D O D$ & ARMY & $0602784 \mathrm{~A}-\mathrm{M}$ Thary Engineering Technolog. & AT40 - Mobility \& Weapons Effects Technotogy & DA359211 & VIP \\
\hline 143 & $D O D$ & ARMY & $0602784 \mathrm{~A}-\mathrm{M}$ litary Engineening Technolog & AT40 - Mobility \& Weapons Effects Technology & DA359212 & VIP \\
\hline 144 & $D O D$ & ARMY & $0602784 \mathrm{~A}-$ Miltary Engineening Technolog & AT40 - Mobility \& Weapons Effects Technology & DA359214 & VIP \\
\hline 145 & $D O D$ & ARMY & $0601102 A$ - Defense Research Sciences & BT25 - Environmental Research - Corps of Engi & DAAH049310293 & E/G \\
\hline 146 & $D O D$ & ARMY & 0601102A - Defense Research Sciences & A305 - Automatic Target Recognition Research & DAAH049310307 & $E / G$ \\
\hline 147 & $D O D$ & ARMY & 0601102A - Defense Research Sciences & AH42-Materials and Mechanios & DAAH049320012 & E/CA \\
\hline 148 & DOD & ARMY & $0601102 A$-Defense Research Sciences & BSO4 - Military Pcllutants and Heath Hazards & DAAH049320018 & $E / G$ \\
\hline 149 & $D O D$ & DAA & BMDO/0603218C - Research and Support & Project number unspecified & DC900027 & $E / C$ \\
\hline 150 & DOD & Airforce & $0602201 F$ - Aerospace Flight Dynamics & 2402 - Vehicle Equipment & DF120491 & $E / C$ \\
\hline 151 & $D O D$ & DAA & OSD/0601103D - University Research Initia & Project number unspecified & DF290017 & $E / G$ \\
\hline 152 & $D O D$ & DAA & OSD/0602228D - Historically Black Collun & Project number unspecified & DF290117 & $E / C$ \\
\hline 153 & $D O D$ & Airforce & $0601102 F$ - Defense Research Sciences & 2303 - Chemistry & DF291785 & $E / C$ \\
\hline 154 & $D O D$ & Airforce & $0601102 F$ - Defense Research Sciences & 2303-Chemistry & DF291790 & $E / G$ \\
\hline 155 & $D O D$ & Airforce & $0602202 \mathrm{~F}$ - Human Systems Techrology & 7184 - Human System Interface Technologies & DF316985 & $E / C$ \\
\hline 156 & $D O D$ & Airforce & $0602202 \mathrm{~F}$ - Human Systems Technology & 6302 - Occurational and Environmental Toxic H & DF318045 & VIP \\
\hline 157 & $D O D$ & Airforce & $0602202 \mathrm{~F}$ - Human Systems Technology & 7231 - Safety and Aircrew Effectiveness in Mect & DF319395 & $E / C$ \\
\hline 158 & $D O D$ & Airforce & $0602202 \mathrm{~F}$ - Human Systems Technology & 7184 - Human System Interface Tectinologies & DF319575 & EIC \\
\hline 159 & DOD & Aifforce & $0603106 \mathrm{~F}$ - Logistios Systems Technology & 2745 - Logisties for Contingency Operations and & DF320035 & $E / C$ \\
\hline 160 & DOD & Airforce & $0605502 \mathrm{~F}$-Small Business Innovative Res & Projeci number unspecified & DF322865 & E/C \\
\hline 161 & DOD & AirForce & $0602601 F$ - Advanced Weapons & Project number unspecified & DF344652 & VIP \\
\hline 162 & DOD & Airforce & $0602302 \mathrm{~F}$ - Rocket Propulsion \& Astronaut ti & 3059 - MISSILE SYSTEMS PROPULSION TEC & DF345923 & $E / C$ \\
\hline 163 & $D O D$ & Airforce & $0601101 \mathrm{~F}$ - In-House Laboratory Independe & Projed number unspecified & DF406004 & VIIP \\
\hline 164 & $D O D$ & Airforce & $0603723 F$ - Civil \& Environmental Engineer & 2104 - Air Base Operability Advanced Technoto & DF406006 & I/IP \\
\hline 165 & DOD & Airforce & $0602206 \mathrm{~F}$ - Civil Engineering \& Environmer & 2673 - Air Base Operability Technology & DF406012 & NS \\
\hline 166 & DOD & Airforce & $0603723 \mathrm{~F}$ - Civil \& Environmental Engineer & 2103 - Environmental Quality Advanced Techno & DF407330 & $E / C$ \\
\hline 167 & $D O D$ & Airforce & $0603723 \mathrm{~F}$ - Civil \& Environmental Engineer & 2103 - Environmental Quality Advanced Techno & DF407334 & NS \\
\hline 168 & $D O D$ & Airforce & $0602601 F$ - Advanced Weapons & Project number unspecified & DF407335 & VIP \\
\hline 169 & $D O D$ & Airforce & $0601101 \mathrm{~F}$ - In-House Laboratory independe & Project number unspecified & DF407349 & VIP \\
\hline 170 & DOD & Airforce & $0603723 \mathrm{~F}$ - Civil \& Envisonmental Engineer & 2104 - Air Base Operability Advanced Technolo & DF407354 & NS \\
\hline 171 & DOD & Airforce & $0603723 \mathrm{~F}$ - Civil \& Environmental Engineer & 2104 - Air Base Operability Actuanced Technolo & DF407367 & $E / C$ \\
\hline 172 & DOD & Airforce & $0603723 \mathrm{~F}$ - Civil \& Environmental Engineer & 2104 - Air Base Operability Advanced Technolo & CDF407383 & IIIP \\
\hline 173 & $D O D$ & Airforce & $0605502 \mathrm{~F}$ - Small Business Innovative Res & Project number unspecified & DF407456 & NS \\
\hline 174 & DOD & Airforce & $0605502 \mathrm{~F}$ - Small Business Innovative Res & Projed number unspocified & DF 407463 & $E / C$ \\
\hline 175 & DOD & Airforce & $0602201 \mathrm{~F}$ - Aerospace Flight Dynamics & Project number unspecified & DF407465 & NS \\
\hline 176 & $D O D$ & Airforce & $0602206 \mathrm{~F}$ - Civil Engineering \& Environmer & 2673-Air Base Operabifify Technology & DF407469 & NS \\
\hline 177 & $D O D$ & Airforce & $0602206 \mathrm{~F}$ - Civil Engineering \& Environmer & 2673 - Air Base Operability Technology & DF407471 & NS \\
\hline 178 & $D O D$ & Airforce & $0602206 \mathrm{~F}$ - Civil Engineering \& Environmer & 2673 - Air Base Operability Technology & DF407474 & VIIP \\
\hline 178 & DOD & Airforce & $0602206 \mathrm{~F}$ - Civit Engineering \& Environmer & 2673- Air Base Operability Technology & DF407477 & VIIP \\
\hline 180 & $D O D$ & DAA & BMDO/0603218C - Research and Support & 1601 - INNOVATIVE SCIENCE AND TECHNO & DF450012 & $E / C$ \\
\hline 181 & DOD & DAA & BMDO/0603218C - Research and Suppon & 1601 - INNOVATIVE SCIENCE AND TECHNO & DF450987 & $E / C$ \\
\hline 182 & $D O D$ & DAA & BMDO/0603218C - Research and Support & Project number unspecified & DF451107 & $E / C$ \\
\hline 183 & $D O D$ & DAA & BMDO/0603218C - Research and Support & Project number unspocified & DF451108 & $E / C$ \\
\hline 184 & DOD & Airforce & $0603112 \mathrm{~F}$ - Advanced Materials for Weapor & 2100 - Laser Hardened Materials & DF500010 & $E / C$ \\
\hline 185 & $D O D$ & Airforce & $0602102 \mathrm{~F}$-Matenals & 2418 - Metalic Structurad Malerials & DF501744 & $E / C$ \\
\hline 186 & DOD & AirForce & $0601102 F$-Defense Research Sciences & Zac2 - Solid Nechanics and Structures & DF516590 & $E / C$ \\
\hline 187 & DOD & DAA & BMDOY0603218C - Research and Suppon 1 & 1601 - INNOVATIVE SCIENCE AND TECHNO & DF525650 & $E / G$ \\
\hline 188 & $D O D$ & DAA & BMDO/0603217C - Ballistic Missile Defense & 1503-Power \& Power Condit & DF527123 & $E / C$ \\
\hline & $D O D$ & Airforce & $0602601 \mathrm{~F}$ - Advanced Weapons & Project number unspecified & DF527945 & $E / C$ \\
\hline 190 & $D O D$ & Airforce & $0602601 \mathrm{~F}$ - Advanced Weapons & 5797 - Advanced Weapons Technology and A & $\mathrm{DF} 528026$ & $E / C$ \\
\hline 191 & $D O D$ & Airforce & $0602601 F$ - Advanced Weapons & 5797 - Advanced Weapons Technology and Ast & DF528044 & $E / C$ \\
\hline 192 & DOD & Airforce & $0602601 F$ - Advanced Weapons & 5797 - Advanced Waapons Technology and A & DF528045 & $E / C$ \\
\hline 193 & $D O D$ & DAA & ARPA $0602708 \mathrm{E}$ - Integrated Command \& & Project number unspecified & DF553102 & $E / C$ \\
\hline 194 & $D O D^{-}$ & DAA & ARPA $0603739 \mathrm{E}$ - Mariufacturirig Technola & Project number unspecified & DF663681 & $E / C$ \\
\hline 195 & DOD & $\overline{D A A}$ & ARPA $0603739 \mathrm{E}$ - Manufacturing Technola & Project number unspecified & DF664312 & $E / C$ \\
\hline
\end{tabular}




\begin{tabular}{|c|c|c|c|c|c|c|c|c|c|c|c|c|c|}
\hline & Agency & $\begin{array}{l}\text { Start } \\
\text { Date }\end{array}$ & $\begin{array}{l}\text { End } \\
\text { Date }\end{array}$ & $\begin{array}{l}\text { Place of } \\
\text { Performance }\end{array}$ & 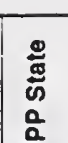 & Performer Name & \begin{tabular}{c|c} 
Perf & $=\bar{c}$ \\
Type & $\bar{\Phi}$ \\
\end{tabular} & $\begin{array}{c}\text { Total } \\
\text { Award } \\
\text { Amount }\end{array}$ & $\begin{array}{c}\text { Average } \\
\text { Annual } \\
\text { Funding }\end{array}$ & $\begin{array}{l}\text { FY } \\
\text { Total } \\
\text { Amt }\end{array}$ & $\begin{array}{c}\text { FY Fod } \\
\text { Amt }\end{array}$ & \begin{tabular}{c|} 
FY \\
Non- \\
Fod \\
Armt
\end{tabular} & Prod. \\
\hline 131 & $D O D$ & Oct-91 & Sep-96 & & & NO PERFORMER INFO AV & NotSpec & & 212.5 & 250 & 250 & & 7 \\
\hline 132 & $D O D$ & Oct-91 & Sep-99 & & & NO PERFORMER INFO AV & NotSpec & & 245.5 & 206 & 206 & & 7 \\
\hline 133 & 000 & act-92 & Sep-93 & Vicksburg & & OCE WATERWAYS EXPEA & FedGdARMY & ENGINEA & 33.333 & 0 & 0 & & 7 \\
\hline 134 & DOD & Aug-93 & Oct-95 & Fort Worth & $\mathrm{TX}$ & NO PERFORMER INFO AV & NotSpoc & 692 & 230.667 & 100 & 100 & & 7 \\
\hline 135 & $D O D$ & Oct-89 & May-95 & & & NO PERFORMER INFO AV & NotSpec & & 48.75 & 20 & 20 & & \\
\hline 136 & $D O D$ & Doc-90 & Feb-94 & Herts & & HUDSON (PROF.J A) 0707 & Oth HUDS & 126 & 31.5 & & & & 7 \\
\hline 137 & $D O D$ & Mar-92 & May-95 & Huntsville & $\overline{A L}$ & NO PERFORMER INFO AV & NOISPOC & 12753 & 4063.5 & 3326 & 3326 & & 7 \\
\hline 138 & $D O D$ & Doc-93 & Nov-95 & Huntington Bea & $C A$ & NO PERFORMER INFO AV & NotSpoc & 620 & 620 & 620 & 620 & & 7 \\
\hline 139 & $D O D$ & Apr-93 & Jun-96 & Aalborg & & DEPT OF CIVIL ENGINEEA & PriEdIAALBC & 48 & $\frac{12}{12}$ & & & & $\frac{6}{6}$ \\
\hline 140 & DOD & Oct-93 & Sep-97 & & & NO PERFORMER INFO AV & NotSpec & & 300 & 100 & 100 & & \\
\hline 141 & DOD & Oct-93 & Sep-95 & & & NO PERFORMER INFO AV & NotSpoc & & 187.5 & 175 & 175 & & 7 \\
\hline 142 & $D O D$ & Oct-93 & Sep-01 & & & NO PERFORMER INFO AV & NotSpec & & 172.5 & 100 & 100 & & 8 \\
\hline 143 & $D O D$ & Oct-93 & Sep-96 & & & NO PERFORMER INFO AV II & NotSpec & & 169 & 150 & 150 & & 7 \\
\hline 144 & $D O D$ & Oct-92 & Sep-95 & & & NO PERFORMER INFO AV & NotSpec & & 109.667 & 50 & 50 & & 7 \\
\hline 145 & $D O D$ & Sep-93 & Aug-96 & New Brunswick & $\mathrm{NJ}$ & RUTGERS, THE STATE UN & Edlnst & & 126.677 & & & & 7 \\
\hline 146 & $D O D$ & Sep-93 & Aug-96 & Boulder & co & REGENTS OF UNIV OF CO & Edlnst & & 105.83 & & & & 7 \\
\hline 147 & $D O D$ & Sep-93 & Sep-95 & Greensboro & NC & NORTH CAROLINA A\&T UN & Edlnst & & 416 & & & & 7 \\
\hline 148 & $D O D$ & Sep-93 & Sep-95 & Greensboro & NC & NORTH CAROLINA A\&T UM & Edlnst & & -150 & 150 & 150 & 0 & 7 \\
\hline 149 & $D O D$ & Jul-90 & Jut-95 & Reston & VA & NO PERFORMER INFO AV & NotSpec & 8252 & 1305 & 1305 & 1305 & & 7 \\
\hline 150 & $D O D$ & Nov-92 & May-96 & Newport Beach & $C A$ & NO PERFORMER INFO AV & NotSpec & 265 & 66.25 & & & & 7 \\
\hline 151 & DOD & Aug-93 & OCt-94 & Newark & & UNIV OF DELAWARE & EdInS DELAV & 140 & 46.667 & & & & 7 \\
\hline 152 & DOD & Sep-93 & Nov-94 & Hoboken & & STEVENS INSTITUTE OF TI & PriEd|STEVE & 448 & 149.333 & & & & 7 \\
\hline 153 & $D O D$ & Apr-94 & Jun-96 & & & NO PERFORN & NotSpec & 249 & 83 & & & & 7 \\
\hline 154 & $D O D$ & May-94 & Jun-96 & & & NO PERFORI & NotSpec & 235 & 78.333 & & & & 7 \\
\hline 155 & $D O D$ & Dec-89 & Dec-94 & Dayton & $\mathrm{OH}$ & NO PERFOR & NotSpec & 1282 & 302.667 & 254 & 254 & & 5 \\
\hline 156 & DOD & Feb-92 & Dec-96 & & & NO PERFORN & NotSpec & & 75.25 & 76 & 76 & & 7 \\
\hline 15 & $D O D$ & Oct-91 & Oct-97 & Dayton & $\mathrm{OH}$ & NO PERFORMER INFO AV & NotSpec & 799 & 179.667 & 210 & 210 & & 5 \\
\hline 158 & $D O D$ & Oât-93 & Apr -98 & Dayion & $\mathrm{OH}$ & NO PERFORME & NotSpec & 111 & 111 & 111 & 111 & & 5 \\
\hline 159 & DOD & Oct-92 & Sep-97 & Wright Patterso & $\mathrm{OH}$ & NO PERFORME & NotSpec & 9425 & 1885 & & & & 7 \\
\hline 160 & $D O D$ & May-93 & Jul-94 & San Antonio & $T X$ & NO PERFOR & NotSpec & 96 & 96 & & & & 7 \\
\hline 161 & DOD & May-88 & Jun-95 & & & NO PERFOR & NotSpec & & 4 & & & & 7 \\
\hline 162 & $D O D$ & Dec-86 & May-95 & Brigham City & UT & NO PERFOR & NotSpec & & 422 & 422 & 422 & & 7 \\
\hline 163 & $D O D$ & Apr-90 & Jan-94 & & & NO PERFORI & NotSpec & 55 & 18333.5 & & & & 7 \\
\hline 164 & DOD & Mar-92 & Mar-95 & & & NO PERFORMER INFO AV & NotSpec & 86 & 86 & & & & 7 \\
\hline 165 & $D O D$ & Feb-92 & Dec-94 & McLean & & SAME & IndBu $\overline{B D M C}$ & 30 & 30 & & & & 7 \\
\hline 166 & $D O D$ & Jan-90 & Mar-94 & Durham & NC & AIR FORCE ENGINEERING & PriEd DUKE & 325 & 62481.33 & 25000 & 25000 & & 7 \\
\hline 167 & $D O D$ & Sep-91 & Nov-94 & Tyndall Air Foro & $\overrightarrow{F L}$ & OAK RIDGE N & PriEdOAK R & 315 & 185 & & & & 7 \\
\hline 168 & $D O D$ & Oct-90 & Oct-94 & & & NO PERFORMER INFO AV, & Notspec & 44 & 44 & 44 & 44 & & 7 \\
\hline 169 & DOD & Apr-90 & Jan-94 & & & NO PERFO & NotSpec & 55 & 18333.5 & & & & 7 \\
\hline 170 & $D O D$ & Jan-91 & Jan-94 & & & NO PERFO & NotSpec & & 50000 & & & & 7 \\
\hline 171 & DOD & Oct-90 & Oct-93 & & & NO PERF & NotSpec & 995 & 300 & & & & 7 \\
\hline 172 & 000 & Mar-92 & Mar-95 & & & NO PERFOF & NotSpec & 86 & 86 & & & & 7 \\
\hline 173 & 000 & Jul-94 & Jut-96 & & & NO PERFOR & NotSpec & 62 & 62 & & & & 7 \\
\hline 174 & $D O D$ & Jun-92 & Dec-92 & Emeryille & CA & NO PERF & NotSpec & 721 & 243.667 & 305 & 305 & & 7 \\
\hline 175 & $D O D$ & Oct-92 & Sep-94 & Alexandria & VA & INSTITUT & NonprIINSTIT. & 100 & 105 & 110 & 110 & & 7 \\
\hline 176 & $D O D$ & Sep-93 & Sep-95 & & & NO PERFOR & NotSpec & 1294 & 431.333 & 433 & 433 & & 7 \\
\hline 177 & $D O D$ & Oct-92 & Oct-95 & Salt Lake City & & UNIVERSITY OF UTAH & EdInSUTAH & UNIV SA & 38333.33 & 65000 & 65000 & & 7 \\
\hline 178 & DOD & Nov-92 & Nov-94 & & & NO PERFORI & NotSpoc & 484 & 161.333 & 149 & 149 & & 7 \\
\hline 179 & DOD & Oct-92 & Oc-95 & & & NO PERF & NotSpoc & & 54500 & 50000 & 50000 & & 7 \\
\hline 180 & DOD & Feb-92 & Jan-96 & Kent & WA & NO PERF & NotSpec & 262 & 52.4 & & & & 7 \\
\hline 181 & DOD & Sep-89 & Oct-94 & San Diego & $C A$ & NO PERFORMER INFO AV & NotSpoc & 655 & 93.571 & & & & 7 \\
\hline 182 & $D O D$ & Aug-92 & Nov-95 & & NV & NO PERFORMER INFO AVII & NotSpec & & & & & & 7 \\
\hline 183 & $D O D$ & Mar-93 & Od-95 & Boul & NV & NO PERFOR & NotSpec & 184 & 46 & & & & 7 \\
\hline 164 & $D O D$ & & & & MO & NO PERFOR & NotSpec & 2229 & 85.5 & & & & 7 \\
\hline 185 & DOD & Apr-93 & Mar-97 & Seattle & WA & NO PERFO & NotSpec & 155 & 31 & & & & 7 \\
\hline 186 & $D O D$ & Mar-92 & May-95 & & & NO PERFOP & & 204 & 51 & & & & 7 \\
\hline & $D O D$ & Jun-93 & Jan-95 & & & NO PERF & NotSpec & 48 & 16 & & & & 7 \\
\hline 188 & DOD & Oct-92 & Sep-96 & & & NOPERFOR & NotSpec & & 350 & 500 & 500 & & 7 \\
\hline 189 & $D O D$ & Aug-93 & Oct-95 & & & NOPERFOR & NotSper, & & 186.333 & 410 & 410 & & 7 \\
\hline 190 & $D O D$ & OCt-93 & $O_{C 1-94}$ & All & NM & NO PERFOR & NotSpec & 8667 & 75 & 75 & 75 & & 7 \\
\hline 191 & $D 0^{\circ}$ & Oct-93 & Oct-94 & & NM & NO PERFOR & NotSpec & 5593 & 70 & 70 & 70 & & 7 \\
\hline & $D O D$ & Oct-93 & Oct-94 & Albuquerque & NM. & NO PERF & NotSpec & 8667 & 27 & 27 & 27 & & 7 \\
\hline 193 & $D O D$ & Jun-91 & Nov-93 & Milpitas & CA & TO PERFORMER INFO AV & Nolsper & 2234 & 558.5 & & & & 7 \\
\hline 194 & $D O D$ & Dea-92 & Der-96 & & & NO PERFORI & NotSpec & & & & & & 7 \\
\hline 195 & $D O D$ & Jut-94 & & & & NO PERFORMER INFO AV & & & & & & & 7 \\
\hline
\end{tabular}




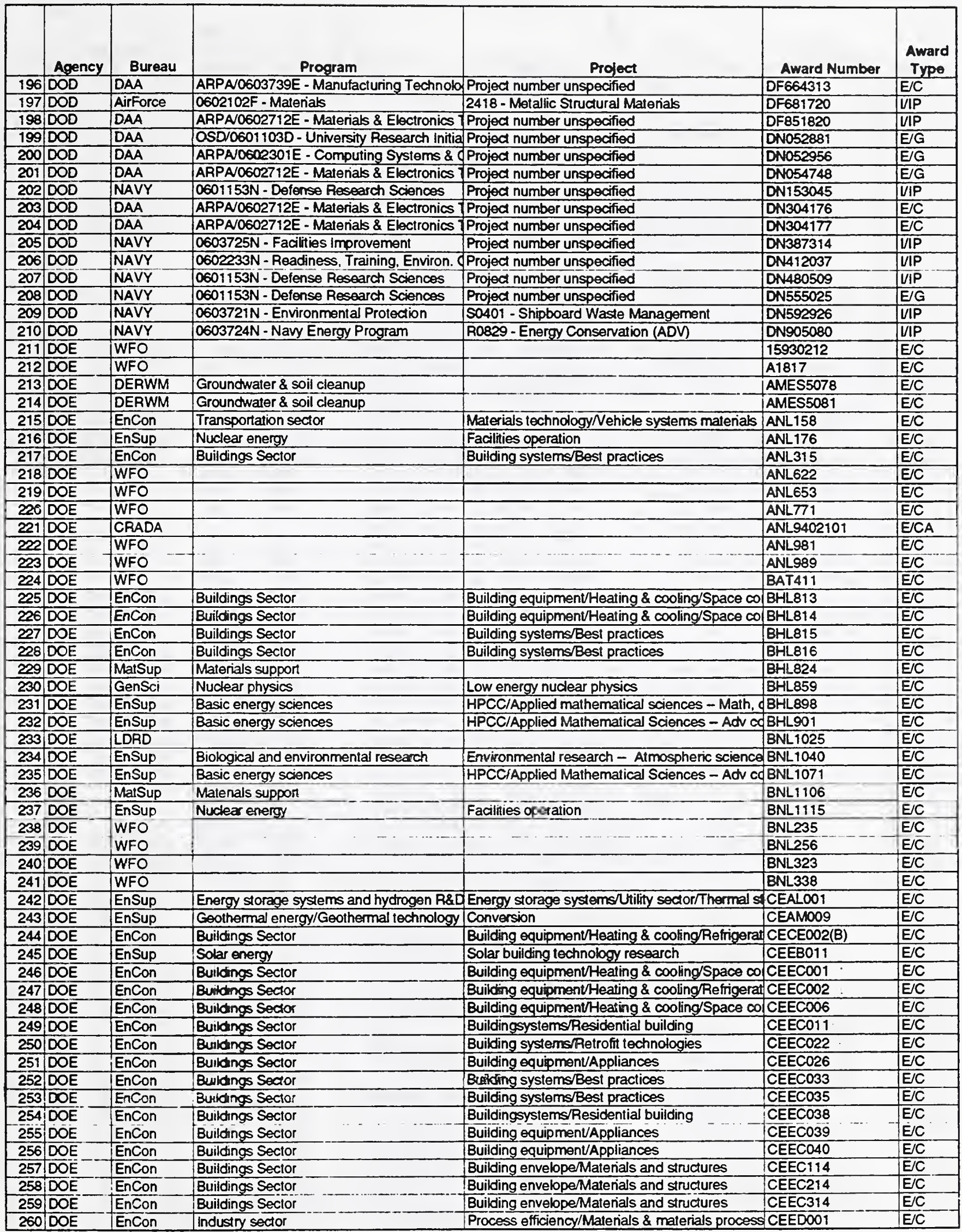




\begin{tabular}{|c|c|c|c|c|c|c|c|c|c|c|c|c|c|}
\hline & Agency & $\begin{array}{l}\text { Start } \\
\text { Date }\end{array}$ & $\begin{array}{l}\text { End } \\
\text { Date }\end{array}$ & $\begin{array}{l}\text { Place of } \\
\text { Performance }\end{array}$ & 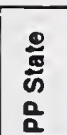 & Performer Name & $\begin{array}{l}\text { Pert } \\
\text { Type } \\
\end{array}$ & $\begin{array}{c}\text { Total } \\
\text { Award } \\
\text { Amount }\end{array}$ & $\begin{array}{l}\text { Avorage } \\
\text { Annual } \\
\text { Funding }\end{array}$ & $\begin{array}{c}\text { FY } \\
\text { Total } \\
\text { Amt }\end{array}$ & $\begin{array}{c}\text { FY Fod } \\
\text { Amt }\end{array}$ & \begin{tabular}{c|} 
FY \\
Non- \\
Fod \\
Amt
\end{tabular} & $\begin{array}{c}\text { Prod. } \\
*\end{array}$ \\
\hline 196 & $D O D$ & Jul-94 & & & & NO PERFORMER INFO AV & NotSpoc & & & & & & 7 \\
\hline 197 & DOD & Od-76 & Sep-95 & Wright-Patterso & In AFE & MLSE MATERIALS ENGINE & FodGdAIR FO & DRCE MAT & TERIALS LA & $A B$ WRIC & GHT-PAT & TERS & 7 \\
\hline 198 & DOD & 0 a -84 & Sop-99 & Wright-Patterso & In AFE & MLLN MATERIALS BEHAVI & FodG AIR FO & RCE MA] & 67.5 & & & & 7 \\
\hline 199 & DOD & Jul-92 & \begin{tabular}{|l|} 
Jun-95 \\
\end{tabular} & Pasadena & & CALIFORNIA INSTITUTE O & PriEd CALIFC & 2170 & 1085 & & & & 7 \\
\hline 200 & $D O D$ & Jul-92 & Jun-95 & & & NO PERFORMER INFO AV & NotSpoc & 1969 & 679.5 & & & & 8 \\
\hline 201 & $D O D$ & May-94 & Apr-97 & Cambridge & & MASSACHUSETTS INSTIT & PriEdIMASS & 2143 & 720 & 773 & 773 & & 7 \\
\hline 202 & $D O D$ & Oct-92 & & Washinglon DC & & NAVAL RESEARCH LABOA & FodG NAVAL & RESEAF & 100 & & & & 7 \\
\hline 203 & $D O D$ & May-94 & May-97 & Ann Arbor & MI & UNIVERSITY OF MICHIGAI & PriEd|MICHIC & 499 & \begin{tabular}{|l|}
13688.33 \\
\end{tabular} & 10231 & 10231 & & 8 \\
\hline 204 & $D O D$ & Apr-94 & Apr-97 & Salh Lake City & UT & UNIVERSITY OF UTAH DE & PriEdUTAH & 2600 & 13688.33 & 10231 & 10231 & & 8 \\
\hline 205 & $D O D$ & Jan-83 & Oct-96 & Port Hueneme & & NAVAL CIVIL ENGINEERIN & FodGCNAVAL & CIVILEI & 152.5 & & & & 8 \\
\hline 206 & $D O D$ & Oct-91 & Sep-95 & & & NO PERFORMER INFO AV & NotSpec & & 135 & & & & 6 \\
\hline 207 & DOD & $0 a-83$ & & Washington $D C$ & & NAVAL RESEARCH LABO & FodGdNAVAL & RESEAA & 299 & & & & 7 \\
\hline 208 & $D O D$ & Nov-88 & act-95 & Seatlle & & UNIVERSITY OF WASHINC & EdIns WASH & 600 & 83.5 & 80 & 80 & & 7 \\
\hline 209 & $D O D$ & Oct-89 & & & & NO PERFORMER INFO AV & NotSpoc & & & & & & 7 \\
\hline 210 & DOD & Oct-84 & & & & NO PERFORMER INFO AV & NotSpec & & & & & & 7 \\
\hline 211 & DOE & & & Albuquerque & & Sandia National Laboratories & NotSpec & 345 & 130.2 & 130.2 & 130.2 & & 7 \\
\hline 212 & DOE & & & Albuquerque & & Sandia National Laboralories & NotSpec & 4500 & 34.3 & 34.3 & 34.3 & & 7 \\
\hline 213 & DOE & & & Ames & & Ames Laboratory & NotSpec & 255 & 255 & 255 & 255 & & 7 \\
\hline 214 & DOE & & & Ames & & Ames Laboratory & NotSpec & 150 & 150 & 150 & 150 & & 7 \\
\hline 215 & $D O E$ & & & Argonne & & Argonne National Laboratory & NotSpec & 6175 & 1008.333 & 1100 & 1100 & & 7 \\
\hline 216 & $D O E$ & & & Argonne & & Argonne National Laborat ory & NotSpec & 25281 & 4827 & 5100 & 5100 & & 7 \\
\hline 217 & DOE & Oct-90 & & Argonne & & Argonne National Laboratory & NotSpec & 33 & 11 & 15 & 15 & & 7 \\
\hline 218 & DOE & $\operatorname{Jan}-93$ & Dec-93 & Argonne & & Argonne National Laboratory & NotSpec & 210 & 210 & & & & 7 \\
\hline 219 & DOE & Dec-91 & \begin{tabular}{|l|} 
Mar-94 \\
\end{tabular} & Argonne & & Argonne National Laboratory & NotSpoc & 4698 & 299 & & & & 7 \\
\hline 220 & DOE & Nov-91 & Aug-95 & Argonne & & Argonne National Laborat on & NotSpec & 500 & 500 & & & & 7 \\
\hline 221 & DOE & Aug-94 & \begin{tabular}{|l|} 
Jun-96 \\
\end{tabular} & Argonne & & Argonne National Laboratory & NotSpec & & & & & & 7 \\
\hline 222 & DOE & Mar-94 & \begin{tabular}{|l|} 
Mar-95 \\
\end{tabular} & Argonne & & Argonne National Laboralory & NotSpec & 50 & 25 & & & & 7 \\
\hline 223 & $D O E$ & Sep-93 & $\operatorname{sep}-94$ & Argonne & & Asgonne National Laboratory & NotSpec & 198 & 99 & & & & 7 \\
\hline 224 & DOE & Jun-94 & Nov-94 & Richland & & Pacific Northwest Laboratory & NotSpec & 26.5 & 13.25 & & & & 7 \\
\hline 225 & DOE & & & Upton & & Brookhaven National Laborat & NotSpec & 3510 & 878.333 & 875 & 875 & & $\frac{6}{7}$ \\
\hline 226 & DOE & & & Upton & & Brookhaven National Laborat & NotSpec & 1054 & 180.667 & 175 & 175 & & 7 \\
\hline 227 & DOE & & & Upion & & Brookhaven National Laborat & NotSpec & 24 & 4.667 & 2 & 2 & & 7 \\
\hline 228 & DOE & & & Upton & & Brookhaven National Laborat & NotSpec & 1022 & 257 & 250 & 250 & & 2 \\
\hline 229 & $D O E$ & & & Upton & & Brookhaven National Laborat & NOASPEC & 1169 & 342.667 & 693 & 693 & & 3 \\
\hline 230 & DOE & & & Upton & & Brookhaven National Laborat & NotSpec & 311 & 155.5 & 151 & 151 & & $\frac{5}{7}$ \\
\hline 231 & DOE & & & Upton & & Brookhaven National Laborat & NotSpec & 1542 & 514 & 570 & 570 & & 7 \\
\hline 232 & DOE & Oct-92 & & Upton & & Brookhaven National Laborat & NotSpec & 1635 & 545 & 598 & 598 & & 7 \\
\hline 233 & $D O E$ & & & Upton & & Brookhaven National Laborat & NotSpec & 100.3 & 100.3 & 100.3 & $\mid 100.3$ & & 7 \\
\hline 234 & $D O E$ & & & Upton & & Brookhaven National Laborat & NotSpec & 1001 & 300.5 & 231 & 231 & & 7 \\
\hline 235 & DOE & & Sep-94 & Upton & & Brookhaven National Laborat & NotSpec & 442 & 442 & 442 & 442 & & 7 \\
\hline 236 & DOE & Oct-93 & & Upton & & Brookhaven National Laborat & NotSpec & 0 & & & & & 7 \\
\hline 237 & DOE & Jun-91 & Sep-94 & Upion & & Brookhaven National Laborat & NotSpec & 0 & 0 & & & & 7 \\
\hline 238 & DOE & & Dec-93 & Upion & & Brookhaven National Laborat & NotSpec & 400 & 400 & & & & -7 \\
\hline 239 & $D O E$ & Dec-93- & $\operatorname{sep}-94$ & Upton & & Brookhaven National Laboral & NotSpec & 80.2 & 80.2 & 80.2 & 80.2 & & 2 \\
\hline 240 & DOE & & Sep-95 & \begin{tabular}{|l|l|l} 
Upton \\
\end{tabular} & & Brookhaven National Laborat & NotSpec & 340 & 340 & 340 & 340 & & $\frac{2}{5}$ \\
\hline 241 & DOE & & Mar-94 & Upton & & Brookhaven National Laborat & NotSpec & 0 & & & & & 5 \\
\hline 242 & DOE & Jul-75 & Sep-93 & Oak Ridge & & Oak Ridge National Laborato & NotSpec & 283 & 283 & 283 & 283 & & $\frac{5}{7}$ \\
\hline 243 & DOE & & & \begin{tabular}{|l} 
Oak Ridge \\
\end{tabular} & & Oak Ridge National Laborato & NotSpec & 843 & 205 & 20 & 20 & & $\frac{1}{7}$ \\
\hline 244 & DOE & Oct-76 & & Oak Ridge & & Oak Ridge National Laborato & NotSpec & 0 & & & & & 7 \\
\hline 245 & $D O E$ & & & Oak Ridge & & Oak Ridge National Laboralo & NotSpec & 296 & 148 & 150 & 150 & & $\frac{7}{7}$ \\
\hline 246 & DOE & Oct-76 & & Oak Ridge & & Oak Ridge National Laborado & NotSpec & 21579 & 5010 & 5049 & 5049 & & 7 \\
\hline 247 & $D O E$ & & & \begin{tabular}{|l} 
Oak Ridge \\
\end{tabular} & & Oak Ridge National Laborato & NotSpec & 4757 & 1543 & 1490 & 1490 & & 7 \\
\hline 248 & DOE & Apr-88 & & Oak Pidge & & Oak Ridge National Laboraid & NotSpec & 957 & 243.667 & 314 & 314 & & 7 \\
\hline 249 & DOE & Dec-86 & & Cak Ridge & & Oak Ridge National Laboralo & NolSpec & 1978 & 484.333 & 433 & 433 & & 1 \\
\hline 250 & DOE & Jan-84 & & Oak Ridge & & Oak Ridge National Laborato & NotSpec & 7134 & 1761.333 & 1821 & 1821 & & 1 \\
\hline 251 & DOE & & & Oak Ridge & & Oak Ridge National Laborald & NoISpec & 15 & 15 & 15 & 15 & & 6 \\
\hline 252 & DOE & Apr-85 & & Oak Ridge & & Oak Ridge National Laborato & NotSpec & 1960 & 441.667 & 540 & 540 & & 7 \\
\hline 253 & DOE & Oct-90 & & Oak Ridge & & Oak Ridge National Laborado & NotSpec & 253 & 114 & 108 & 108 & & $\frac{1}{7}$ \\
\hline 254 & DOE & & & Oak Ridge & & Oak Ridge National Laborato & NotSpec & 263 & 80 & $\pi$ & $\pi$ & & $\frac{1}{7}$ \\
\hline 255 & DOE & & & Oak Ridge & & Oak Ridge National Laborato & NOISpec & 350 & 175 & 235 & 235 & & 7 \\
\hline 256 & DOE & & & Oak Ridge & & Oak Ridge National Laborato & NotSpec & 125 & 125 & 125 & 125 & & $\frac{1}{7}$ \\
\hline 257 & $D O E$ & Oct-79 & & Oak Ridge & & Oak Ridge National Laboralo & NotSpec & 849 & 190 & 238 & 238 & & 1 \\
\hline 258 & DOE & Oct-79 & & Oak Ridge & & Oak Ridge National Laborato & NotSpec & 4330 & 1443.333 & 1245 & 1245 & & 7 \\
\hline 259 & $D O E$ & oct-79 & & Oak Ridge & & Oak Ridge National Laborato & NotSpec & 6838 & 2279.333 & 2358 & 2358 & & $\frac{1}{7}$ \\
\hline$\frac{260}{260}$ & $D O E$ & Oct-90 & & Oak Ridge & & Oak Ridge National Laborato & NotSpec & 15586 & 3528.667 & 3336 & 3336 & & $\frac{1}{7}$ \\
\hline
\end{tabular}




\begin{tabular}{|c|c|c|c|c|c|c|}
\hline & Ageney & Bureau & Program & Project & Award Number & $\begin{array}{l}\text { Award } \\
\text { Type }\end{array}$ \\
\hline 261 & DOE & Encon & Industry soctor & Energy systems/Procoss heating \& cooling/Heal & CEEDO07 & ESC \\
\hline 262 & $D O E$ & Encon & \begin{tabular}{|l|} 
Industry sector \\
\end{tabular} & Energy systems/Process heating \& cooling/Ther & CEED010 & $E / C$ \\
\hline 263 & DOE & Encon & Buildings Sector & Building systems/Best practices & CEWAS44 & $E / C$ \\
\hline 264 & DOE & Encon & Buildings Sector & Builfing envelope/Materials and structures & CEWBE31 & $E / C$ \\
\hline 265 & $\mathrm{DOE}$ & Encon & Buildings Sector & Building envetoperMaterials and structures & CEWBE41 & $E / C$ \\
\hline 266 & DOE & EnCon & Buitdings Sector & Building systems/Best practicos & CEWDO03 & $E / C$ \\
\hline 267 & DOE & Encon & Industry sector & Process efficiency/Materials \& materials process & CEWPI71 & $E / C$ \\
\hline 268 & DOE & MarSup & Materials support & & DPW1222 & $E / C$ \\
\hline 269 & DOE & Encon & Buildings Sector & Building systems/Retrofit technologies & EEWAS 13 & $E / C$ \\
\hline 270 & DOE & Ensup & Environment, Safety \& Health & Health physics and industrial hygiene R\&D & EHW1842 & EIC \\
\hline 271 & DOE & EnSup & Basic energy sciences & Engineoring \& goosciences - Geosciences rese & ERKCES2 & EIC \\
\hline 272 & $\mathrm{DOE}$ & EnSup & Basic energy sciences & HPCC/Applied Mathematical Sciences - Adv Co & ERKCZ12 & E/C \\
\hline 273 & DOE & EnSup & Biological and environmental research & Environmental research - Terrestrial transport & ERKP508 & $E / C$ \\
\hline 274 & DOE & EnSup & Technology transfer & & ERKUCT1 & $E / C$ \\
\hline 275 & DOE & EnSup & Biological and environmental research & Heath effects - Biological research & ERW1828 & $E / C$ \\
\hline 276 & DOE & Encon & Buildings Sector & Building systems/Best practices & ETEC11001 & $E / C$ \\
\hline 277 & $\mathrm{DOE}$ & FE & Petroleum, coal, and gas program & Coal - Advanced research \& lechnology/Maleria & FEAA028 & $E / C$ \\
\hline 278 & DOE & EnSup & Basic energy sciences & Extramural awards & FG0293ER25158 & $E / G$ \\
\hline 279 & DOE & EnSup & Basic energy sciences & Extramural awards & FG0393ER61600 & $E / G$ \\
\hline 280 & DOE & EnSup & Small business innovative research & & FG0594ER81890 & $E / G$ \\
\hline 281 & $D O E$ & EnSup & Nuclear energy & Space reactor power systems/SP-100 space rea & HANFORD 109 & $E / C$ \\
\hline 282 & DOE & MatSup & Nuclear safeguards and security & Technology and system developmenu/full scale & INEL4014 & $E / C$ \\
\hline 283 & $D O E$ & EnSup & Environment, Safety \& Heath & Heatth physics and industrial hygiene R\&D & INEL4108 & $E / C$ \\
\hline 284 & DOE & EnCon & Buildings Sector & Building systems/Best practices & INEL4131 & $E / C$ \\
\hline 285 & DOE & WFO & & & $L-1195 B$ & $E / C$ \\
\hline 286 & DOE & WFO & & & $L-1349$ & $E / C$ \\
\hline 287 & DOE & WFO & & & L-1418 & $E / C$ \\
\hline 288 & $D O E$ & WFO & & & $L-4141$ & EIC \\
\hline 289 & DOE & WFO & & & $L-4544$ & $E / C$ \\
\hline 290 & $\mathrm{DOE}$ & WFO & & & $L 1330$ & $E / C$ \\
\hline 291 & $D O E$ & WFO & & & L1334 & $E / C$ \\
\hline 292 & DOE & CRADA & & & LANLLC9110020 & E/CA \\
\hline 293 & DOE & CRADA & & & LANLLC9210064 & ECA \\
\hline 294 & DOE & Encon & Buildings Sector & Building systems/Best practices & LBL4708 & $E / C$ \\
\hline 295 & DOE & Encon & Buildings Sector & Building systems/Commercial buildings & LBL4712 & E/C \\
\hline 296 & DOE & Encon & Buildings Sector & Building systems/Retrofit technologies & LBL4720 & $E / C$ \\
\hline 297 & DOE & Encon & Buildings Sector & Building equipment/Appliances & LBL4722 & $E / C$ \\
\hline 298 & DOE & Encon & Buildings Sector & Building systems/Best practices & LBL4735 & $E / C$ \\
\hline 299 & DOE & EnCon & \begin{tabular}{|l|} 
Buildings Sector \\
\end{tabular} & Building envelope/Materials and structures & LBL4739 & $E / C$ \\
\hline 300 & DOE & EnCon & \begin{tabular}{|l} 
Buildings Sector \\
\end{tabular} & Building systems/Retrofit technologies & LBL4742 & $E / C$ \\
\hline 301 & DOE & Encon & Buildings Sector & Building envelope/Indoor air quality & LBL4745 & $E / C$ \\
\hline 302 & DOE & Encon & Buildings Sector & Building systems/Commercial buildings & LBL4746 & $E C$ \\
\hline 303 & DOE & Encon & Buildings Sector & Building envelopeWindows and glazings & LBL4747 & $E C$ \\
\hline 304 & DOE & Encon & Butldings Secior & Building equipmenulighting & LBL 4748 & $E \in C$ \\
\hline 305 & DOE & EnCon & Buildings Seclor & Building envelope/Windows and glazings & LBL4749 & EIC \\
\hline 306 & DOE & EnCon & Buildings Sector & Buildingsystems/Residential building & LBL4756 & EC \\
\hline 307 & DOE & Encon & Buildings Sector & Building envelope/Materials and structures & LBL4761 & $E / C$ \\
\hline 308 & DOE & Encon & Buildings Sector & Building systems/Best practices & LBL4778 & $E / C$ \\
\hline 309 & DOE & EnCon & Buildings Sector & Building systems/Best practices & LBL4787 & $E / C$ \\
\hline 310 & DOE & Ensup & Biological and environmental research & Heath effects - Biological research & LBL4789 & $E / C$ \\
\hline 311 & $D O E$ & Encon & Industry sector & Process efficiency/Materials \& materials process & LBL4798 & $E / C$ \\
\hline 312 & DOE & EnCon & Buildings Sector & Building equipmenU/Appliances & LBL4799 & E/C \\
\hline 313 & DOE & CRADA & & & LBLPBG90073 & E/CA \\
\hline 314 & DOE & CRADA & & & LBLPBG9115702 & $E / C A$ \\
\hline 315 & DOE & CRADA & & & LBLPBG92023 & $E / C A$ \\
\hline 316 & DOE & CRADA & & & LBLPBG92027 & $E / C A$ \\
\hline 317 & DOE & CRADA & & & LBLPBG92028 & E/CA \\
\hline 318 & DOE & CRADA & & & LBLPBG 92080 & $E / C A$ \\
\hline 319 & NSF & Medical \&: P & VA Medical Center - (Palo Alto, CA) & & 83718 & $E / G$ \\
\hline 320 & DOE & CRADA & & & LBLPBG93019 & $E / C A$ \\
\hline 321 & DOE & CRADA & & & LBLPBG93040 & E/CA \\
\hline 322 & DOE & CRADA & & & LBLPBG93151 & E/CA \\
\hline$\frac{323}{323}$ & DOE & Encon & Buildings Sector & Building systems/Best practices & LINL01EEW0014 & $E / C$ \\
\hline 324 & DOE & Ensup & Nuclear energy & Space reactor power systems/SP-100 space rea & NEAF 607 & E/C \\
\hline 325 & DOE & Matsup & Nuclear safeguards and security & Technology and system developmenviscience \& & NEW0019 & $E / C$ \\
\hline
\end{tabular}




\begin{tabular}{|c|c|c|c|c|c|c|c|c|c|c|c|c|c|}
\hline & Agency & $\begin{array}{l}\text { Start } \\
\text { Date }\end{array}$ & $\begin{array}{l}\text { End } \\
\text { Date }\end{array}$ & $\begin{array}{c}\text { Place of } \\
\text { Performance }\end{array}$ & $\begin{array}{l}\text { Ĩ } \\
\text { के } \\
a \\
a\end{array}$ & Performer Name & $\begin{array}{l}\text { Perf } \\
\text { Type }\end{array}$ & \begin{tabular}{|c|} 
Total \\
Award \\
Amount
\end{tabular} & $\begin{array}{c}\text { Average } \\
\text { Annual } \\
\text { Funding }\end{array}$ & $\begin{array}{l}\text { FY } \\
\text { Total } \\
\text { Amt }\end{array}$ & $\begin{array}{c}\text { FY Fed } \\
\text { Amt }\end{array}$ & \begin{tabular}{|c|} 
FY \\
Non- \\
Fed \\
Ant
\end{tabular} & $\begin{array}{c}\text { Prod. } \\
*\end{array}$ \\
\hline 261 & DOE & Apr-82 & & Oak Ridge & & Oak Ridge National Laborato & NotSpec & 4753 & 819.333 & 610 & 610 & & 7 \\
\hline 262 & DOE & Oct-89 & & Oak Ridge & & Oak Ridge National Laborato & NotSpec & 2126 & 511.667 & 507 & 507 & & 7 \\
\hline 263 & DOE & Oct-93 & & Golden & & National Renewable Energy & INotSpec & 1425 & 450 & 400 & 400 & & 4 \\
\hline 264 & $D O E$ & Oct-92 & & Golden & & National Renewable Energy & NotSpec & 20840 & 6946.667 & 7040 & 7040 & & 7 \\
\hline 265 & $D O E$ & $04-93$ & & Golden & & National Renewable Energy & INotSpec & 16270 & 5160 & 4920 & 4920 & & 7 \\
\hline 266 & $D O E$ & $0 x-92$ & & Golden & & National Renewable Energy & INotSpec & 3300 & 1100 & 1100 & 1100 & & 7 \\
\hline 267 & $D O E$ & $\operatorname{Jan}-93$ & & Golden & & National Renewable Energy & NotSpec & 2843 & 710.75 & 350 & 350 & & 7 \\
\hline 268 & DOE & $\operatorname{Jan}-92$ & & Oak Ridge & & Oak Ridge Institute for Scien & NotSpec & 1757 & 280.5 & 315 & 315 & & 4 \\
\hline 269 & DOE & Mar-91 & Mar-94 & Golden & & National Renewable Energy l & NotSpec & 112.5 & 112.5 & & & & 6 \\
\hline 270 & DOE & Mar-91 & & Oak Ridge & & Oak Ridge Institute for Scien & NotSpec & 3638 & 757.667 & 882 & 882 & & 3 \\
\hline 271 & DOE & & & Oak Ridge & & Oak Ridge National Laborato & NotSpec & 14 & 14 & 14 & 14 & & 7 \\
\hline 272 & DOE & & & Oak Ridge & & Oak Ridge National Laborato & NotSpec & 4260 & 1420.5 & 1581 & 1581 & & 7 \\
\hline 273 & DOE & & & Oak Ridge & & Oak Ridge National Laborato & NotSpec & 1179 & 367.5 & 300 & 300 & & 7 \\
\hline 274 & DOE & OCt-93 & Sep-94 & Oak Ridge & & Oak Ridge National Laborato & NotSpec & 100 & 100 & 100 & 100 & & 7 \\
\hline 275 & $D O E$ & Jul-87 & & Oak Ridge & & Oak Ridge Institute for Scien & NotSpoc & 727 & 180 & 182 & 182 & & 7 \\
\hline 276 & DOE & Oct-90 & & Canoga Park & & Energy Technology Engineer & NotSpec & 107 & 35.667 & 50 & 50 & & 7 \\
\hline 277 & DOE & Oct-79 & & Oak Ridge & & Oak Ridge National Laborato & NotSpec & 12018 & 4006 & 15 & 15 & & 7 \\
\hline 278 & DOE & Apr-93 & Apr-94 & Albany & NY & NEW YORK STATE UNIV F & EdInst & 700 & 700 & & & & 7 \\
\hline 279 & DOE & May-93 & Apr-96 & Salt Lake City & UT & ROGERS \& ASSOC ENG C & IndBus & 164.145 & $\overline{52.771}$ & 52.214 & 52.214 & 0 & 7 \\
\hline 280 & DOE & Aug-94 & Mar-97 & Albuquerque & $\mathrm{NM}$ & THERMAL ELECTRIC DEV & IndBus & 884.999 & 74.999 & 74.999 & 74.999 & 0 & 7 \\
\hline 281 & DOE & $0 a-83$ & & Richland & & Hanford Site & NotSpec & 7202 & 1590 & 1375 & 1375 & & 7 \\
\hline 282 & DOE & Jan-86 & & Idaho Falls & & Idaho National Engineering L & NotSpec & 3543.7 & 902.35 & 957.7 & 957.7 & & 7 \\
\hline 283 & $D O E$ & Aug-93 & & Idaho Falls & & Idaho National Engineering $L$ & NotSpec & 775 & 187.5 & 185 & 185 & & 3 \\
\hline 284 & DOE & Oct-90 & & Idaho Falls & & Idaho National Engineering $L$ & NotSpec & 70 & 13.5 & 6 & 6 & & 7 \\
\hline 285 & DOE & & & Livermore & & Lawrence Livermore Nationa & NotSpec & 5275.8 & 479.9 & 479.9 & 479.9 & & 7 \\
\hline 286 & DOE & & & Livermore & & Lawrence Livermore Nationa & NotSpec & 1158 & 140.5 & 140.5 & 140.5 & & 5 \\
\hline 287 & DOE & & & Livermore & & Lawrence Livermore Nationa & NotSpec & 88.1 & 81.5 & 81.5 & 81.5 & & 7 \\
\hline 288 & $\overline{D O E}$ & & & Livermore & & Lawrence Livermore Nationa & NotSpec & 4150.8 & $10 \overline{10} \overline{7}$ & 1010.7 & 1010.7 & & 7 \\
\hline 289 & DOE & & & Livermore & & Lawrence Livermoro Nationa & NotSpec & 1997.2 & 0.9 & 0.9 & 0.9 & & 7 \\
\hline 290 & $D O E$ & & & Albuquerque & & Sandia National Laboratories & NotSpec & 458 & 26.2 & 26.2 & 26.2 & & 7 \\
\hline 291 & DOE & & & Albuquerque & & Sandia National Laboratories & NotSpec & 1234 & 24 & 24 & 24 & & 7 \\
\hline 292 & DOE & Jan-92 & Jan-94 & Los Alamos & & Los Alamos National Laborat & INotSpec & & & & & & 7 \\
\hline 293 & DOE & Jan-93 & Jan-95 & Los Alamos & & Los Alamos National Laboral & NotSpec & & & & & & 7 \\
\hline 294 & DOE & Oct-92 & & Berkeley & & Lawrence Berkeley Laboralor & NotSpec & 1815 & 632.5 & 730 & 730 & & 7 \\
\hline 295 & DOE & JuL-74 & & Berkeley & & Lawrence Berkeley Laborator & NotSpec & 462 & 152 & 150 & 150 & & 7 \\
\hline 296 & DOE & Oct-84 & & Berkeley & & Lawrence Berkeley Laborator & NotSpec & 381 & 100.667 & 75 & 75 & & 7 \\
\hline 297 & DOE & Jun-79 & & Berkeley & & Lawrence Berkeley Laborator & NotSpec & 11409 & 3749 & 3697 & 3697 & & 7 \\
\hline 298 & $\overline{D O E}$ & Oct-86 & & Berkeley & & Lawrence Berkeley Laborator & NotSpec & 4605 & 1300 & 650 & 650 & & 7 \\
\hline 299 & DOE & Apr-91 & & Berkeley & & Lawrence Berkeley Laborato & NotSpec & 409 & 101 & 100 & 100 & & 7 \\
\hline 300 & $D O E$ & May-77 & & Berkeley & & Lawrence Berkeley Laborato & NotSpec & 1285 & 308.333 & 225 & 225 & & 7 \\
\hline 301 & DOE & May-77 & & Berkeley & & Lawrence Berkeley Laborator & NotSpec & 3600 & 1200 & 1200 & 1200 & & 7 \\
\hline 302 & DOE & May-77 & & Berkeley & & Lawrence Berkeley Laboralor & NotSpec & 3570 & 822 & 950 & 950 & & 7 \\
\hline 303 & DOE & May-77 & & Berkeley & & Lawrence Berkeley Laborato & Noispec & 9755.2 & 2326.733 & 2625 & 2625 & & $\overline{7}$ \\
\hline 304 & DOE & Jui-76 & & Berkeley & & Lawrence Berkeley Laborator & NotSpec & 10717 & 2638 & 2650 & 2650 & & 7 \\
\hline 305 & DOE & Oct -90 & & Berkeley & & Lawrence Berkeley Laborafor & NotSpec & 2179 & 726.333 & 745 & 745 & & 7 \\
\hline 306 & DOE & Jun-92 & & Berkeley & & Lawrence Berkeley Laborato & NotSpec & 253 & 66.667 & 50 & 50 & & 7 \\
\hline 307 & DOE & Apr-91 & & Berkeley & & Lawrence Berkeley Laborato & NotSpec & 156 & 52 & 52 & 52 & & 7 \\
\hline 308 & DOE & Oct-90 & & Berkeley & & Lawrence Berkeley Laborato & NotSpec & 3071 & 1009 & 995 & 995 & & 7 \\
\hline 309 & DOE & Jan-88 & & Berkeley & & Lawrence Berkeley Laborato & NotSpec & 1225 & 302.667 & 300 & 300 & & 7 \\
\hline 310 & DOE & Jul-76 & & Berkeley & & Lawrence Berkeley Laborato & NotSpec & 1704 & 426.333 & 399 & 399 & & 7 \\
\hline 311 & DOE & Aug-91 & & Berkeley & & Lawrence Berkeley Laborato & NotSpec & 800 & 266.667 & 250 & 250 & & 7 \\
\hline 312 & DOE & Jul-74 & & Berkeley & & Lawrence Berkeley Laborato & NotSpec & 321.2 & 107.067 & 107 & 107 & & 7 \\
\hline 313 & DOE & Dec-92 & Dec-93 & Berkeley & & Lawrence Berkeley Laborator & NotSpec & & & & & & 7 \\
\hline 314 & DOE & Jun-93 & Jun-94 & Berkeley & & Lawrence Berkeley Laborato & NotSpoc & 61.798 & 30.899 & & & & 7 \\
\hline 315 & DOE & Dec-92 & Dec-93 & Berkeley & & Lawrence Berkeley Laborato & NotSpec & & & & & & 7 \\
\hline 316 & $D O E$ & Deo-92 & Dec-93 & Berkeley & & Lawrence Berkeley Laborator & NotSpec & & & & & & 7 \\
\hline 317 & $D O E$ & Dec-92 & Dec-93 & Berketey & & Lawrence Berkeley Laborator & NotSpec & & & & & & 7 \\
\hline 318 & DOE & Dea-92 & Fet-94 & Berkeley & & Lawrence Berkeley Laborator & NotSpec & & & & & & 7 \\
\hline 319 & NSF & Oct-92 & $\operatorname{sep}-94$ & Palo Allo & & VA Modical Center - (Palo Al & FedGov & & 7.697 & 0 & 0 & & 7 \\
\hline 320 & DOE & Aug-93 & Áug-94 & Borkeley & & Lawrence Berkeley Laborator & NotSpec & & & & & & 7 \\
\hline 321 & $D O E$ & Mar-94 & Jun-95 & Berkeley & & Lawrence Berkeley Laborator & NotSpec & & & & & & 7 \\
\hline 322 & $D O E$ & Jun-93 & Oct-93 & Berkeley & & Lawrence Berkeley Laborator & NotSpec & & & & & & 7 \\
\hline 323 & $D O E$ & Oct-91 & & Livermore & & Lawrence Livermore Nationa & NotSpec & 60 & 15 & 15 & 15 & & 7 \\
\hline 324 & $D O E$ & Oct-85 & & Oak Ridge & & Oak Ridge National Laborato & NotSpec & 1318 & 659 & 371 & 371 & & 7 \\
\hline 325 & $D O E$ & & & Livermore & & Lawrence Livermore Nationa & NotSpec & 1600 & 533.333 & 600 & 600 & & $\overline{7}$ \\
\hline
\end{tabular}




\begin{tabular}{|c|c|c|c|c|c|c|}
\hline & Agency & Bureau & Program & Project & Award Number & $\begin{array}{l}\text { Award } \\
\text { Type }\end{array}$ \\
\hline 326 & DOE & CRADA & & & NREL_92004 & EJCA \\
\hline 327 & DOE & CRADA & & & NREL92008 & E/CA \\
\hline 328 & $D O E$ & EnSup & Environment. Safety \& Health & Health physics and industrial hygiene R\&D & ORISE101.5 & $E / C$ \\
\hline 329 & DOE & WFO & & & ORNL16132 & $E / C$ \\
\hline 330 & DOE & WFO & & & ORNL16367 & $E / C$ \\
\hline 331 & DOE & CRADA & & & ORNL910054 & $E / C A$ \\
\hline 332 & DOE & CRADA & & & ORNL910063 & $\mathrm{E} / \mathrm{CA}$ \\
\hline 333 & DOE & CRADA & & & ORNL920116 & E/CA \\
\hline 334 & DOE & CAADA & & & ORNL920123 & E/CA \\
\hline 335 & DOE & CRADA & & & ORNL920134 & E/CA \\
\hline 336 & DOE & CRADA & & & ORNL930173 & $E / C A$ \\
\hline 337 & DOE & CRADA & & & ORNL930191 & $E / C A$ \\
\hline 338 & DOE & CRADA & & & ORNL930192 & $\mathrm{E} / \mathrm{CA}$ \\
\hline 339 & $D O E$ & CRADA & & & ORNL930196 & $\mathrm{E} / \mathrm{CA}$ \\
\hline 340 & $D O E$ & CRADA & & & ORNL930229 & E/CA \\
\hline 341 & DOE & CRADA & & & ORNL930234 & $E / C A$ \\
\hline 342 & DOE & CRADA & & & ORNL930235 & $E / C A$ \\
\hline 343 & $D O E$ & CRADA & & & ORNL940263 & $E / C A$ \\
\hline 344 & DOE & CRADA & & & ORNL940278 & E/CA \\
\hline 345 & DOE & EnSup & Fusion energy & Magnetic fusion energy - Confinement sys $/$ Toka & $P 6009$ & $E / C$ \\
\hline 346 & DOE & EnSup & Fusion energy & Magnetic fusion energy - Development \& technd & P6030 & E/C \\
\hline 347 & DOE & CRADA & & & PNL029 & E/CA \\
\hline 348 & DOE & CRADA & & & PNL036 & E/CA \\
\hline 349 & DOE & EnSup & Environment, Safety \& Health & Health physics and industrial hygiene R\&D & PNL1044 & $E \bar{C}$ \\
\hline 350 & DOE & DERWM & Pollution prevention & & PNL1111 & $E / C$ \\
\hline 351 & DOE & DERWM & Pollution prevention & & PNL1113 & $E / C$ \\
\hline 352 & DOE & DERWM & Pollution prevention & & PNL1114 & $E / C$ \\
\hline 353 & $\overline{D O E}$ & EnSup & Technology transfer & & PNL 1129.5 & $\mathrm{E} / \mathrm{C}$ \\
\hline 354 & DOE & LDRD & & & PNL1155 & $E / C$ \\
\hline 355 & $\overline{D O E}$ & LDRD & & & PNL1177 & $E / C$ \\
\hline 356 & DOE & LDRD & & & PNL1207 & E/C \\
\hline 357 & DOE & LDRD & & & PNL1240 & $E / C$ \\
\hline 358 & DOE & LDRD & & & PNL1269 & $E / C$ \\
\hline 359 & DOE & EnSup & Biological and environmental research & Environmental research - Terrestrial transport & PNL551 & $E / C$ \\
\hline 366 & DOE & EnSup & Biological and environmental research & Environmental research - Terrestrial transport & PNL552 & $E / C$ \\
\hline 361 & $\overline{D O E}$ & EnSup & Biological and environmental research & Environmental research - Terrestrial transport & PNL555 & $E / C$ \\
\hline 362 & DOE & CRADA & & & PNL810 & $E / C A$ \\
\hline 363 & DOE & EnCon & Buildings Sector & Building envelope/Windows and glazings & PNL639 & $E / C$ \\
\hline 364 & DOE & MatSup & Nuclear safoguards and security & Technology and system developmenU Science \& & PNL642 & $E / C$ \\
\hline 365 & DOE & LDRD & & & PNL551 & $E / C$ \\
\hline 366 & DOE & LDRD & & & PNL732 & $\mathrm{E} / \mathrm{C}$ \\
\hline 367 & DOE & DERWM & Groundwater \& soil cleanup & & PNL795 & $E / C$ \\
\hline 368 & DOE & DERWM & Groundwater \& soil cleanup & & PNL796 & $E / C$ \\
\hline 369 & DOOE & DERWM & Groundwater \& soil cleanup & & PNL 804 & $\mathrm{E} / \mathrm{C}^{-}$ \\
\hline 370 & DOE & DERWM & Waste retrieval \& processing & & PNL806 & $E / C$ \\
\hline 371 & DOE & DERWM & Waste relrieval \& processing & & PNL807 & $E / C$ \\
\hline 372 & DOE & DERWM & Waste rotrieval \& processing & & PNL810 & E/C \\
\hline 373 & $D O E$ & DERWM & Waste retrieval \& procossing & & PNL810 & $E / C$ \\
\hline 374 & DOE & DERWM & Waste retrieval \& processing & & PNL810 & $E / C$ \\
\hline 375 & $D O E$ & DERWM & Waste retrieval \& processing & & PNL822 & $E / C$ \\
\hline 370 & $\overline{D O E}$ & DERWM & Groundwater \& soil cleanup & & PNL825 & $\mathrm{E} / \mathrm{C}$ \\
\hline 377 & $D O E$ & DERWM & Groundwater \& soil cleanup & & PNL830 & $E / C$ \\
\hline 378 & DOE & Encon & Buildings Sector & Building envelopenwindows and glazings & PNL854 & E/C \\
\hline 379 & DOE & Encon & Buildings Sector & Building systems/Best practices & PNL855 & $E / C$ \\
\hline 380 & DOE & EnCon & Industry sector & Process efficiency/Malerials \& materials procoss & PNL858 & $E / C$ \\
\hline 381 & DOE & DERWM & Groundwater \& soil cleanup & & PNL869 & $E / C$ \\
\hline 382 & DOE & DERWM & Oher activities & & RWW0009 & $E / C$ \\
\hline 383 & DOE & LDRD & & & SNL1089 & EJC \\
\hline 384 & DOE & LDRD & & & SNL1121 & $E / C$ \\
\hline 385 & $D O E$ & DERWM & Groundwater \& soil cleanup & & SNL1385 & $E / C$ \\
\hline 386 & $D O E$ & Encon & Industry sector & Process efficiency/Materials \& materials process & SNL1387 & $E / C$ \\
\hline 387 & DOE & Ensup & Nuclear energy & Light water reactors/Advanced light waler reacto & SNL1544 & $E / C$ \\
\hline 388 & $D O E$ & DERTWM & Other activities & & SNL1562 & E/C \\
\hline 389 & DOE & DERWM & Groundwaler \& soil cleanup & & SNL1630 & E/C \\
\hline 390 & $\overline{D O E}$ & DERWM & Groundwaler \& soil cleanup & & SNL1632 & $E / C$ \\
\hline
\end{tabular}




\begin{tabular}{|c|c|c|c|c|c|c|c|c|c|c|c|c|c|}
\hline & Agency & $\begin{array}{l}\text { Start } \\
\text { Date }\end{array}$ & $\begin{array}{l}\text { End } \\
\text { Date }\end{array}$ & $\begin{array}{c}\text { Place of } \\
\text { Performance }\end{array}$ & $\begin{array}{l}\stackrel{9}{9} \\
\text { c } \\
a \\
a\end{array}$ & Performer Name & $\begin{array}{l}\text { Perf } \\
\text { Type }\end{array}$ & $\begin{array}{c}\text { Total } \\
\text { Award } \\
\text { Amount }\end{array}$ & $\begin{array}{l}\text { Average } \\
\text { Annual } \\
\text { Funding }\end{array}$ & $\begin{array}{c}\text { FY } \\
\text { Total } \\
\text { Amt }\end{array}$ & $\begin{array}{c}\text { FY Fed } \\
\text { Amt }\end{array}$ & $\begin{array}{l}\text { FY } \\
\text { Non- } \\
\text { Fed } \\
\text { Amt } \\
\end{array}$ & $\begin{array}{c}\text { Prod. } \\
*\end{array}$ \\
\hline 326 & DOE & Jan-92 & Jan-94 & Golden & & National Renewable Energy & NotSpec & & & & & & 7 \\
\hline 327 & DOE & Aug-92 & Aug-94 & Golden & & National Renowable Energy & NotSpec & & & & & & 7 \\
\hline 328 & DOE & Jan-92 & & Oak Ridge & & Oak Ridge Institute for Scien & NotSpec & 34 & 17 & & & & 7 \\
\hline 329 & DOE & Jul-93 & Aug-96 & Oak Ridge & & Oak Ridge National Laborato & NotSpec & 290.6 & 72.65 & & & & 7 \\
\hline 330 & DOE & Jut-93 & Aug-96 & Oak Ridge & & Oak Ridge National Laborato & NotSpec & 1190 & 500 & 500 & 500 & & 7 \\
\hline 331 & DOE & Sep-93 & & Oak Ridge & & Oak Ridge National Laborato & NotSpec & & & & & & 7 \\
\hline 332 & DOE & Fab-92 & Oct-94 & Oak Ridge & & Oak Ridge National Laborato & NotSpec & & & & & & 6 \\
\hline 333 & DOE & Oct-92 & 0a-95 & Oak Ridge & & Oak Ridge National Laboraio & NotSpec & & & & & & 7 \\
\hline 334 & DOE & Sep-93 & Jut-95 & Oak Ridge & & Oak Ridge National Laborato & NotSpec & & & & & & 7 \\
\hline 335 & DOE & Jun-93 & Jun-94 & Oak Ridge & & Oak Ridge National Laborado & NotSpec & & & & & & 7 \\
\hline 336 & DOE & Aug-93 & Aug-95 & Oak Ridge & & Oak Ridge National Laborato & NotSpec & & & & & & 7 \\
\hline 337 & $\overline{D O E}$ & Sep-93 & Sep-96 & Oak Pidge & & Oak Ridge National Laborato & NotSpec & & & & & & 7 \\
\hline 338 & DOE & $\tan -94$ & Jul-95 & Oak Ridge & & Oak Ridge National Laborato & NotSpec & & & & & & 7 \\
\hline 339 & DOE & Nov-93 & Nov-94 & Oak Ridge & & Oak Ridge National Laborato & NotSpec & & & & & & 7 \\
\hline 340 & DOE & Dec-93 & Dec-94 & Oak Ridge & & Oak Ridge National Laborato & NotSpec & & & & & & 7 \\
\hline 341 & DOE & Apr-94 & Apr-95 & Oak Ridge & & Oak Ridge National Laborato & NotSpec & & & & & & 7 \\
\hline 342 & DOE & Apr-94 & Apr-95 & Oak Ridge & & Oak Ridge National Laborato & NotSpec & & & & & & 7 \\
\hline 343 & DOE & Nay-94 & Feb-95 & Oak Pidge & & Oak Ridge National Laborato & NotSpec & & & & & & 7 \\
\hline 344 & DOE & Aug-94 & Aug-95 & Oak Pidge & & Oak Ridge National Laborato & NotSpec & & & & & & 7 \\
\hline 345 & DOE & Oct-92 & & Princeton & & Princelon Plasma Physics La & NotSpec & 3824.1 & 3824.1 & & & & 7 \\
\hline 346 & DOE & Oct-92 & & Princeton & & Princoton Plasma Physics La & NotSpec & 705.9 & 235.3 & 300.4 & 300.4 & & 7 \\
\hline 347 & IDOE & Jun-93 & & Richland & & Pacific Northwest Laboratory & NotSpec & & & & & & 7 \\
\hline 348 & DOE & Jun-93 & & Richland & & Pacific Northwest Laboratory & NotSpec & & & & & & 7 \\
\hline 349 & DOE & Oct-93 & & Richland & & Pacific Northwest Laboratory & NotSpec & 200 & 200 & & & & 3 \\
\hline 350 & DOE & Sep-94 & Sep-97 & Richland & & Pacific Northwest Laboratory & NotSpec & 117) & 117 & 117 & 117 & & 7 \\
\hline 351 & DOE & Mar-93 & Mar-98 & Richland & & Pacific Northwest Laboratory & NotSpec & 2234 & 744.667 & 812 & 812 & & 7 \\
\hline 352 & $\overline{D O E}$ & Oct-93 & & Richland & & Pacific Northwest Laboratory & NotSpec & 3868 & 1289.333 & 506 & 506 & & 7 \\
\hline 353 & DOE & $\operatorname{sun}-93$ & & Richland & & Pacific Northwest Laboratory & NotSpec & 50326 & 11163 & 9026 & 9026 & & 7 \\
\hline 354 & DOE & & & Richland & & Pacific Northwest Laboratory & NotSpec & 466 & 155.333 & 221 & 221 & & 7 \\
\hline 355 & DOE & & & Richland & & Pacific Northwest Laboratory & NotSpec & 361 & 105.5 & 86 & 86 & & 7 \\
\hline 356 & DOE & & & Richland & & Pacific Northwest Laboratory & NotSpec & 561.8 & 137.267 & 229 & 229 & & 7 \\
\hline 357 & DOE & & & Richland & & Pacific Northwest Laboratory & Notspec & 90 & 45 & 45 & 45 & & 7 \\
\hline 358 & DOE & & & Richland & & Pacific Northwest Laboratory & NotSpec & 813.4 & 203.35 & 85 & 85 & & 7 \\
\hline 359 & DOE & OCt-88 & & Richland & & Pacific Northwest Laboratory & NotSpec & 1315 & 328.75 & 400 & 400 & & 7 \\
\hline 360 & DOE & act-88 & Feb-94 & Richland & & Pacific Northwest Laboratory & NotSpec & 640 & 213.333 & 200 & 200 & & 7 \\
\hline 361 & DOE & Apr-90 & & Richland & & Pacific Northwest Laboratory & NotSpec & 1147 & 286.75 & 320 & 320 & & 7 \\
\hline 362 & DOE & Feb-93 & Sep-95 & Richland & & Pacific Northwest Laboratory & NotSpec & & & & & & 7 \\
\hline 363 & DOE & Mar-93 & Dec-93 & Richland & & Pacific Northwest Laboratory & NotSpec & 374 & 187 & & & & 7 \\
\hline 364 & DOE & Oat-92 & & Richland & & Pacific Northwest Laboratory & NotSpec & 2200 & 2200 & & & & 7 \\
\hline 365 & DOE & Oct-92 & Sep-95 & Richland & & Pacific Northwest Laboratory & NotSpec & 170 & 42.5 & 50 & 50 & & 7 \\
\hline 366 & $D O E$ & Oa-92 & Sep-95 & Richland & & Pacific Northwest Laboratory & NotSpec & 584 & 194.667 & 130 & 130 & & 7 \\
\hline 367 & $D O E$ & & & Richland & & Pacific Norttwest Laboratory & NotSpec & 5948 & 1004.5 & 1460 & 1460 & & 7 \\
\hline 368 & DOE & Apr-93 & & Richland & & Pacific Northwest Laboratory & NotSpec & 2395 & 598.75 & 785 & 785 & & 7 \\
\hline 369 & DOE & Oct-92 & & Richland & & Pacific Northwest Laboratory & NotSpec & 3032 & 379.75 & 546 & 546 & & 8 \\
\hline 370 & DOE & & & Richland & & Pacific Northwest Laboratory & NotSpec & 11625 & 2706.25 & 4100 & 4100 & & 7 \\
\hline 371 & DOE & & & Richland & & Pacific Northwest Laboratory & NotSpec & 16074 & 2214.25 & 3080 & 3080 & & 7 \\
\hline 372 & DOE & & & Richland & & Pacific Northwest Laboratory & NotSpec & 3195 & 611.667 & 630 & 630 & & 7 \\
\hline 373 & DOE & Oct-92 & Sep-94 & Richland & & Pacific Northwest Laboratory & NotSpec & 190 & 190 & & & & 8 \\
\hline 374 & DOE & Oct-92 & & Richland & & Pacific Northwest Laboratory & NotSpec & 1925 & 483.333 & 600 & 600 & & 7 \\
\hline 375 & DOE & Oct-92 & & Richland & & Pacific Northwest Laboratory & NotSpec & 2530 & 843.333 & 900 & 900 & & 7 \\
\hline 376 & DOE & Oct-92 & & Richland & & Pacific Northwest Laboratory & NotSpec & 900 & 450 & 625 & 625 & & 7 \\
\hline 377 & DOE & & & Richland & & Pacific Northwest Laboratory & NotSpec & 1306 & 326.5 & 404 & 404 & & 7 \\
\hline 378 & $\overline{D O E}$ & Mar-93 & Mar-94 & Richland & & Pacific Northwest Laboratory & NotSpec & 1850 & 550 & 500 & 500 & & 7 \\
\hline 379 & DOE & Mar-84 & & Richiand & & Pacific Northwest Laboraiory & NotSpec & 9151 & 2325.5 & 1651 & 1651 & & 7 \\
\hline 380 & DOE & Apr-92 & & Richland & & Pacific Nonthwest Laboratory & NotSpec & 1659 & 414.75 & 807 & 807 & & 7 \\
\hline 381 & DOE & & & Fichland & & Pacific Northwest Laboratory & NotSpec & 190 & 190 & 190 & 190 & & 7 \\
\hline 382 & DOE & & & Livermore & & Lawrence Livermore National & NotSpec & 450 & 150 & 150 & 150 & & 7 \\
\hline 383 & DOE & & & Albuquerque & & Sandia National Laboratories & NotSpec & 734 & 367 & 314 & 314 & & 7 \\
\hline 384 & DOE & & & Albuquerque & & Sandia National Laboratories & NotSpec & 424 & 212 & 212 & 212 & & 7 \\
\hline 395 & DOE & Mar-89 & Sep-95 & Albuquerque & & Sandia National Laboralories & NotSpec & 40 & 40 & 40 & 40 & & 7 \\
\hline 386 & DOE & Jun-89 & & Albuquerque & & Sandia National Laboratories & NotSpec & 661 & 661 & 661 & 661 & & 7 \\
\hline 387 & DOE & Aug-81 & Sep-99 & Albuquerque & & Sandia National Laboratories & NotSpec & 340 & 340 & 340 & 340 & & 7 \\
\hline 388 & DOE & Jul-91 & Sep-95 & Albuquerque & & Sandia National Laboratories & NotSpec & 24 & 24 & 24 & 24 & & 7 \\
\hline 389 & DOE & Aug-92 & Sep-96 & Albuquerque & & Sandia National Laboratories & NotSpec & 117 & 117 & 117 & 117 & & 7 \\
\hline 390 & DOE & Aug-92 & Sep-95 & Albuquerque & & Sandia National Laboratories & NotSpec & 514 & 514 & 514 & 514 & & 7 \\
\hline
\end{tabular}




\begin{tabular}{|c|c|c|c|c|c|c|}
\hline & Agency & Bureau & $\begin{array}{c}\text { Program } \\
\end{array}$ & Project & Award Number & $\begin{array}{c}\text { Award } \\
\text { Type }\end{array}$ \\
\hline 391 & DOE & DERWM & Grouncwater \& soil cleanup & & SNL1635 & $E / C$ \\
\hline 392 & DOE & DERWM & Other activities & & SNL1636 & $E / C$ \\
\hline 393 & DOE & DERWM & Groundwater \& soil cleanup & & SNL1638 & $E / C$ \\
\hline 394 & DOE & Encon & Buildings Sector & Building systems/Best practicos & SNL1697 & $E / C$ \\
\hline 395 & DOE & DERWM & Groundwater \& soil cleanup & & SNL1712 & $E / C$ \\
\hline 396 & DOE & Encon & Buildings Sector & Building systems/Best practices & SNL1762 & $E / C$ \\
\hline 397 & $D O E$ & LDRD & & & SNL494 & $E / C$ \\
\hline 398 & DOE & LDRD & & & SNL518: = & $E / C$ \\
\hline 399 & DOE & CRADA & & & SNLSC9401229 & $E / C A$ \\
\hline 400 & DOE & CRADA & & . & SRSCR93003 & E/CA \\
\hline 401 & DOE & CRADA & & & SRSCR93010 & E/CA \\
\hline 402 & $D O E$ & DERWM & Groundwater \& soil cleanup & & SRTC 8016 & $E / C$ \\
\hline 403 & DOE & DERWM & Groundwater \& soil cleanup & & SRTC8020 & E/C \\
\hline 404 & DOE & DERWM & Groundwater \& soil cleanup & & SRTC8021 & $E / C$ \\
\hline 405 & DOE & DERWM & Groundwater \& soil cleanup & & SRTC8022 & $E / C$ \\
\hline 406 & $D O E$ & DERWM & Groundwater \& soil cleanup & & SRTC 8024 & $E / C$ \\
\hline 407 & DOE & DERWM & Groundwater \& soil deanup & & SRTC8026 & $E / C$ \\
\hline 408 & DOE & DERWM & Groundwater \& soil cleanup & & SRTC8046 & $E / C$ \\
\hline 409 & DOE & DERWM & Groundwater \& soil cleanup & & SRTC8052 & E/C \\
\hline 410 & DOE & DERWM & Groundwater \& soil cleanup & & SRTC 8057 & $E / C$ \\
\hline 411 & DOE & DERWM & Groundwater \& soil cleanup & & SRTC8059 & $E / C$ \\
\hline 412 & DOE & DERWM & Groundwater \& soil cleanup & & SRTC 8060 & $E / C$ \\
\hline 413 & DOI & MMS & & & CT1090001N1155 & VIP \\
\hline 414 & DOI & BR & & & CTIN0138 & VIP \\
\hline 415 & DOS & OJP & & & $91 \mathrm{JCX} \times 012$ & $E / G$ \\
\hline 416 & DOS & OJP & & & $93 \mathrm{JC} \times 0053$ & $E \bar{G}$ \\
\hline 417 & DOT & FHA & Highway research, development \&technolog & Safety & CT1080017N0497 & VIP \\
\hline 418 & DOT & FHA & Highway research, development \&lechnolog & Materials & CT1 $1080018 \mathrm{NO} 497$ & VIP \\
\hline 419 & DOT & FHA & Highway research, development \&technolog & Pavements & CT1080019N0497 & VIP \\
\hline 420 & DOT & FHA & Highway research, development \&technolod & Structures & CT1080020N0497 & I/IP \\
\hline 421 & DOT & FHA & Highway research, development \&technolog & Environment & CT1080021N0497 & VIP \\
\hline 422 & DOT & FHA & Highway research, development \&lechnolog & Right-of-Way & CT1080022N0497 & VIP \\
\hline 423 & DOT & FHA & Highway research, development \&technoloc & Policy & CT1080023N0497 & VIP \\
\hline 424 & DOT & FHA & Highway research, development \& technolog & Planning & CT1080024N0497 & VIP \\
\hline 425 & DOT & FHA & Highway research, development \&technolog & Molor carrier & CT1080025N0497 & VIP \\
\hline 426 & DVA & M\&PR & VA Medical Center - (San Antonio. TX) & & 77782 & $E / C$ \\
\hline 427 & DVA & M\&PR & VA Medical Center - (Decatur, GA) & & 82943 & VIP \\
\hline 428 & DVA & M\&PR & VA Medical Center - (Decatur, GA) & & 90814 & I/IP \\
\hline 429 & EPA & $R \& D$ & Multimedia research & Lab and field expenses & CTINO433 & VIP \\
\hline 430 & EPA & R\&D & Air quality & & $\mathrm{R} 817083017$ & $E / G$ \\
\hline 431 & EPA & R\&D & Air quality & & R817083018 & E/G \\
\hline 432 & EPA & R\&D & Air quality & & R81708301A & $E / G$ \\
\hline 433 & EPA & R\&D & Air quality & 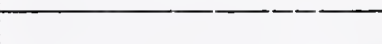 & R81708301C & $E / G$ \\
\hline 434 & EPA & R\&D & Air quality & & $\mathrm{R} 81708301 \mathrm{D}$ & $E / G$ \\
\hline 435 & EPA & R\&D & Air quality & & $R 820299010$ & $E / G$ \\
\hline 436 & EPA & R\&D & Air quality & & R820299012 & $E / G$ \\
\hline 437 & EPA & $R \& D$ & Air quality & & R820299013 & $E / G$ \\
\hline 438 & EPA & R\&D & Multimedia research & Research \& development & $R 820755010$ & $E / G$ \\
\hline 439 & EPA & $R \& D$ & Multimedia research & Research \& development & R820755012 & $E / G$ \\
\hline 440 & EPA & R\&D & Air quality & & R822356011 & $E / G$ \\
\hline 441 & EPA & R\&D & Air quality & & $R 822870010$ & $E / G$ \\
\hline 442 & EPA & R\&D & Air quality & & R822870011 & $E / G$ \\
\hline 443 & EPA & R\&D & Air quality & & R822870012 & $E / G$ \\
\hline 444 & EPA & RED & Multimedia research & Research \& development & R822957010 & $E / G$ \\
\hline 445 & EPA & R\&D & Air quality & & $R 823632010$ & $E / G$ \\
\hline 446 & EPA & $R \& D$ & Air quality & & $R 823633010$ & $E / G$ \\
\hline 447 & EPA & $R \& D$ & Air quality & & 5992096010 & $E / G$ \\
\hline 446 & EPA & $R \& D$ & Air quality & & $\$ 992100010$ & $E / G$ \\
\hline 449 & EPA & $R \& D$ & Air quality & & X995615010 & $E / G$ \\
\hline 450 & $\mathrm{HHS}$ & $C D C P$ & Natl Institute for Occupational Safety \& Healt & & $0000000 \mathrm{CR} 310097$ & Extramu: \\
\hline 451 & HHS & CDCP & Natl Institute for Occupational Safety \& Healt & & $000000 \mathrm{~K} 10 \mathrm{H} 00087$ & Extramur \\
\hline 452 & $\mathrm{HHS}$ & CDCP & Natl Institute for Occupational Safety \& Healt & & $000000 \mathrm{~K} 10 \mathrm{H} 00107$ & Extramur \\
\hline 453 & $\mathrm{HHS}$ & CDCP & Natl Institute for Occupational Safety \& Healt & & $000000 \mathrm{~K} 10 \mathrm{H} 00110$ & Extramur \\
\hline 454 & $\mathrm{HHS}$ & CDCP & Natl Institute for Occupational Safety \& Healt & & $000000 \mathrm{~K} 10 \mathrm{H} 02103$ & Extramur \\
\hline 455 & $\overline{H H S}$ & $\overline{C D C P}$ & Natl Institute for Ocoupational Safety \& Healt & & $000000 \mathrm{R} 1 \mathrm{OH} 00835$ & Exiramur \\
\hline
\end{tabular}




\begin{tabular}{|c|c|c|c|c|c|c|c|c|c|c|c|c|c|}
\hline & Agency & $\begin{array}{l}\text { Start } \\
\text { Date }\end{array}$ & $\begin{array}{l}\text { End } \\
\text { Date }\end{array}$ & $\begin{array}{c}\text { Place of } \\
\text { Performance }\end{array}$ & $\begin{array}{l}\frac{9}{\$} \\
\text { के } \\
\frac{a}{a}\end{array}$ & Performer Name & Perf $\mid \frac{\bar{E}}{\Phi}$ & $\begin{array}{c}\text { Total } \\
\text { Award } \\
\text { Amount }\end{array}$ & $\begin{array}{c}\text { Average } \\
\text { Annual } \\
\text { Funding }\end{array}$ & $\begin{array}{c}\text { FY } \\
\text { Total } \\
\text { Amt }\end{array}$ & $\begin{array}{c}\text { FY Fod } \\
\text { Amtt }\end{array}$ & \begin{tabular}{|c|} 
FY \\
Non- \\
Fod \\
Amt
\end{tabular} & Prod. \\
\hline 391 & DOE & Nov-92 & Sep-95 & Albuquerque & & Sandia National Laboratories & NotSpec & 196 & 196 & 196 & 196 & & 7 \\
\hline 392 & DOE & Aug-93 & Sep-95 & Albuquerque & & Sandia National Laboratories & NotSpec & 256 & 256 & 256 & 256 & & 7 \\
\hline 393 & DOE & Sep-93 & Sep-00 & Albuquerque & & Sandia National Laboratories & NotSpec & 205 & 205 & 205 & 205 & & 7 \\
\hline 394 & DOE & Jut-94 & Sep-95 & Albuquerque & & Sandia National Laboratories & NotSpec & 16 & 16 & 16 & 16 & & 7 \\
\hline 395 & DOE & Jan-93 & & Albuquarque & & Sandia National Laboratories & NotSpec & 560 & 560 & 560 & 560 & & 7 \\
\hline 396 & DOE & Aug-94 & Sep-95 & Albuquerque & & Sandia National Laboratories & NotSpec & 0 & 0 & & & & 7 \\
\hline 397 & DOE & Oct-92 & & Albuquerque & & Sandia National Laboralories & NotSpec & 1064 & 252 & 212 & 212 & & 7 \\
\hline 398 & DOE & Oct-91 & & Albuquerque & & Sandia National Laboralories & NotSpec & 1208 & 402.667 & 400 & 400 & & 8 \\
\hline 399 & DOE & Sep-94 & Sep-96 & Albuquerque & & Sandia National Laboratorios & NotSpec & & & & & & 7 \\
\hline 400 & DOE & Fab-94 & Fob-95 & Aiken & & Savannah River Technology & NotSpec & & & & & & 7 \\
\hline 401 & DOE & Jan-94 & Oct-94 & Aiken & & Savannah River Technology & NotSpoc & & & & & & 7 \\
\hline 402 & DOE & & & Aiken & & Savannah River Technology & NotSpec & 1000 & 333.333 & 100 & 100 & & 7 \\
\hline 403 & DOE & & & Aiken & & Savannah River Technology & NotSpec & 2425 & 808.333 & 800 & 800 & & 7 \\
\hline 404 & DOE & & & Aiken & & Savannah River Technology & NotSpec & 2870 & 956.667 & 720 & 720 & & 7 \\
\hline 405 & DOE & & & Aiken & & Savannah River Technology & NotSpec & 2670 & 667.5 & 700 & 700 & & 7 \\
\hline 406 & DOE & & & Aiken & & Savannah River Technology & NotSpec & 3920 & 730 & 500 & 500 & & 7 \\
\hline 407 & DOE & & & Aiken & & Savannah River Technology & NotSpec & 1175 & 391.667 & 350 & 350 & & $\overline{7}$ \\
\hline 408 & DOE & Oct-92 & & Aiken & & Savannah River Technology & NotSpec & 975 & 487.5 & 125 & 125 & & 7 \\
\hline 409 & DOE & Oct-92 & & Aiken & & Savannah River Technology & iNotSpec & 3575 & 558.333 & 550 & 550 & & 8 \\
\hline 410 & $D O E$ & Oct-91 & & Aiken & & Savannah River Technology & iNotSpec & 1328 & 442.667 & 100 & 100 & & 7 \\
\hline 411 & DOE & Oct-91 & & Aiken & & Savannah River Technology & NotSpec & 1875 & 937.5 & 720 & 720 & & 7 \\
\hline 412 & DOE & Oct-92 & & Aiken & & Savannah River Tectnology & NotSpec & 1400 & 700 & 500 & 500 & & 7 \\
\hline$\frac{416}{413}$ & DOI & Oct-92 & Sep-94 & & & Risk Reduction Engineering t & NotSpec & & & & & & $\frac{1}{7}$ \\
\hline 414 & DOI & Oct-92 & Sep-94 & & & Engineering and Research LA & NotSpec & & & & & & 7 \\
\hline 415 & DOS & Sep-91 & Nov-93 & Annapolis & MD & KNAPEL, CAROLE & Privindiv & & 246.721 & & & & 6 \\
\hline 416 & DOS & Nov-93 & Apr-95 & Capitola & $\mathrm{CA}$ & KNAPEL, CAROLE & Privindiv & & 109 & & & & 6 \\
\hline 417 & DOT & Oct-92 & Sep-94 & & & Tumer-Fairbank Highway Re: & NotSpec & & & & & & 7 \\
\hline 418 & DOT & $0 \mathrm{ar}-92$ & $\operatorname{sep}-94$ & & & Tumer-Faibank Highway Re & NotSpec & & & & & & 7 \\
\hline 419 & DOT & Oct-92 & Sep-94 & & & Tumer-Fairbank Highway Re & NotSpec & & & & & & 7 \\
\hline 420 & DOT & Oat-92 & Sep-94 & & & Tumer-Fairbank Highway Re & NotSpec & & & & & & 7 \\
\hline 421 & DOT & Oct-92 & Sep-94 & & & Tumer-Fairbank Highway Re & NotSpec & & & & & & 7 \\
\hline 422 & DOT & Oct-92 & Sep-94 & & & Tumer-Faibank Highway Re & NotSpec & & & & & & 7 \\
\hline 423 & DOT & Oct-92 & Sep-94 & & & Tumer-Faibank Highway Re & NotSpec & & & & & & 7 \\
\hline 424 & DOT & act-92 & Sep-94 & & & Tumer-Faibank Highway Re & NotSpec & & & & & & 7 \\
\hline 425 & DOT & Oct-92 & Sep-94 & & & Tumer-Fairbank Highway Re & NotSpec & & & & & & 7 \\
\hline 426 & DVA & Oct-92 & Sep-94 & San Antonio & & VA Modical Center - (San An & FedGov & & 4.184 & 3.379 & 3.379 & & 7 \\
\hline 427 & DVA & Oct-92 & Sep-94 & Decatur & & VA Medical Center - (Decatu & FedGor & & 57.981 & 0 & 0 & & $\frac{1}{5}$ \\
\hline 428 & DVA & act-92 & Sep-94 & Decatur & & VA Medical Center - (Decatu) & FedGov & & 62.279 & 72.981 & 72.981 & & 5 \\
\hline 429 & EPA & Oct-92 & Sep-94 & & & Risk Reduction Engineering & NotSpec & & & & & & 7 \\
\hline 430 & EPA & Aug-91 & Aug-94 & Durham & NC & RESEARCH TRIANGLE INS & NonproNonEC & & 416.805 & & & & 7 \\
\hline 431 & EPA & Aug-91 & Aug-94 & Durham & NC & RESEARCH TRIANGLE INS & NonprointonEd & & 603.295 & & & & 7 \\
\hline 432 & EPA & Aug-91 & Aug-94 & Dustram & NC & RESEARCH TRIANGLE INS & NonprofiNonEd & & 68.833 & 70.237 & 68.833 & 1.404 & 7 \\
\hline 433 & EPA & Aug-91 & Aug-95 & Durham & NC & RESEARCH TRIANGLE INS & NonproNonEd & & 94.011 & 95.93 & 94.011 & 1.919 & 7 \\
\hline 434 & EPA & Aug-91 & Aug-95 & Durham & NC & RESEARCH TRIANGLE INS & NonproiñonEd & & 61.5 & 62.756 & 61.5 & 1.256 & 7 \\
\hline 435 & EPA & Oct-92 & Od-95 & Urbana & IL & UNIVERSITY OF ILLINOIS & Edinst & & 91.317 & & & & $\frac{1}{7}$ \\
\hline 436 & EPA & Oct-92 & Oct-94 & Champaign & $\frac{L}{I L}$ & UNIVERSITY OF ILLINOIS & Edinst & & 140 & 151.39 & 140 & 11.39 & $\frac{1}{7}$ \\
\hline 437 & EPA & Oct-92 & Od-94 & Champaign & \begin{tabular}{|l|l} 
IL \\
\end{tabular} & UNIVERSITY OF ILLINOIS & Edlnst & & 20 & 20.993 & 20 & 0.993 & 7 \\
\hline 436 & EPA & Oct-92 & Mar-94 & Ames & $\frac{1 L}{|A|}$ & IOWA STATE UNIVERSITY & StalocGov & & 500 & & & & $\frac{1}{7}$ \\
\hline 439 & EPA & Oct-92 & Mar-94 & Ames & IA & IOWA STATE UNIVERSITY & StalocGov & & 228 & & & & 7 \\
\hline 440 & EPA & Oct-93 & Sep-96 & College Park & MD & UNIV. OF MARYLAND AT d & Edinst & & 133.94 & 140.99 & 133.94 & 7.05 & 7 \\
\hline 441 & EPA & Aug-94 & Jut-96 & Durham & NC & RESEARCH TRIANGLE INS & NonprofNonEd & & 257.992 & 263.26 & 257.99 & 5.266 & 7 \\
\hline 442 & EPA & Aug-94 & Jut-96 & Dutham & NC & RESEARCH TRIANGLE INE & NonprointonEo & & 35 & 35.714 & 35 & 0.714 & 7 \\
\hline 443 & EPA & Aug-94 & Jul-96 & Durham & $N C$ & RESEARCH TRIANGLE INS & NonprofNonEd & & & & & & 7 \\
\hline 444 & EPA & Oct-94 & Oct-96 & Columbus & $\mathrm{OH}$ & BATTELLE MEMORIAL INS & Nonpr BATTEL & LLE MEM & 74.996 & 78.944 & 74.996 & 3.948 & 7 \\
\hline 445 & EPA & Oct-94 & Sep-96 & Minneapolis & MN & UNIVERSITY OF MINNESC & Edlnst & & 10 & 15.657 & 10 & 5.657 & 7 \\
\hline 446 & EPA & Oct-94 & Sep-95 & Atlanta & GA & ASHRAEI & NonproNonEd & & 40 & 81.9 & 40 & 41.9 & 7 \\
\hline 447 & EPA & Sep-94 & Asto-95 & Sermmit & NU & N.J. GRP. AGNST. SMOKMN & NonprofiNonEd & & 16.892 & 17.892 & 16.892 & 1 & $\frac{1}{7}$ \\
\hline 448 & EPA & oct-94 & Sep-95 & Hackensack & NS & AMERICAN CANCER SOCI & NonproNonEd & & 6.585 & 10.985 & 6.585 & 4.4 & 7 \\
\hline 449 & EPA & Sep-94 & Aug-95 & Springfield & IL & ILLINOIS DEPT OF PUBLIC & StalocGor & & 10 & 50.015 & 10 & 40.02 & 7 \\
\hline 450 & HHS & Sep-93 & Sep-94 & Solomons & $M D$ & RAMAZZINI INST FOR OCD & IndBus & & 49.989 & & & & 7 \\
\hline 451 & HHS & May-93 & May-94 & Baltimore & $M D$ & JOHNS HOPKINS UNIVERS & PriEdlnst & & $-\frac{5}{54}$ & & & & 7 \\
\hline 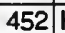 & HHS & Sep-92 & Aug-94 & Madison & WI & UNIVERSITY OF WISCONS & Edlnst & & 53.692 & & & & 7 \\
\hline 453 & HHS & Sep-92 & Sep-94 & Cambridge & MA & HARVARD UNIVERSITY PA & PriEdInst & & 54 & & & & 7 \\
\hline 454 & $\mathrm{HHS}$ & Apr-93 & Jun-94 & Chapel Hill & NC & UNIVERSITY OF NORTH C & EdInst & & 53.936 & & & & 7 \\
\hline 455 & HHS & Sep-92 & Aug-94 & New Orleans & LA & LOUISIANA STATE UNIVEA & Edlnst & & 86.599 & & & & 7 \\
\hline
\end{tabular}




\begin{tabular}{|c|c|c|c|c|c|c|}
\hline & Agency & Bureau & Program & Project & Award Number & $\begin{array}{c}\text { Award } \\
\text { Type }\end{array}$ \\
\hline 456 & HHS & CDCP & \multicolumn{2}{|l|}{ Natl Institute for Occupational Safely \& Health } & $000000 R 10+10257 t$ & Extramur \\
\hline 457 & HHS & CDCP & \multicolumn{2}{|l|}{ Natl Institute for Occupational Safely \& Health } & $000000 \mathrm{R} 10 \mathrm{H} 02664$ & Extramur \\
\hline 458 & $\mathrm{HHS}$ & CDCP & \multicolumn{2}{|l|}{ Natl Institute for Occupation al Safely \& Health } & $000000 R 10+102741$ & Extramur \\
\hline 459 & HHS & CDCP & \multicolumn{2}{|l|}{ Natl Institute for Occupational Safety \& Health } & $000000 R 10+102758$ & Extramun \\
\hline 460 & HHS & COCP & \multicolumn{2}{|l|}{ Natl Institute for Occupational Safety \& Health } & $000000 R_{10} 0+02767$ & Extramur \\
\hline 461 & HHS & CDCP & \multicolumn{2}{|l|}{ Nall Institute for Occupational Safety \& Health } & $000000 \mathrm{R} 1 \mathrm{OH} 02772$ & Extramun \\
\hline 462 & HHS & CDCP & \multicolumn{2}{|l|}{ Nail Institute for Occupational Safety \& Health } & $000000 R 10+102804$ & Extramut \\
\hline 463 & HHS & CDCP & \multicolumn{2}{|l|}{ Nall Institute for Occupational Safely \& Health } & $000000 R_{10}+102858$ & Extramut \\
\hline 464 & HHS & CDCP & \multicolumn{2}{|l|}{ Natl Institute for Occupeational Safety \& Health } & $000000 R 10+102872$ & Extramun \\
\hline 465 & HHS & CDCP & \multicolumn{2}{|l|}{ Natl Institute for Occupational Safety \& Health } & $000000 R 10+102885$ & Extramu \\
\hline 466 & $\mathrm{HHS}$ & CDCP & \multicolumn{2}{|l|}{ Natl Institute for Occupational Safety \& Health } & $000000 R 10+102922$ & Extramur \\
\hline 467 & $\mathrm{HHS}$ & CDCP & \multicolumn{2}{|l|}{ Natl Institute for Occupational Safely \& Health } & $000000 R 10+102948$ & Extramun \\
\hline 468 & HHS & CDCP & \multicolumn{2}{|l|}{ Natl Institute for Occupational Safety \& Health } & $000000 R 10 H 02967$ & Extramur \\
\hline 469 & HHS & CDCP & Natl Institute for Occupational Safety \& Heal & & $000000 R 10+103021$ & Extramu \\
\hline 470 & $\mathrm{HHS}$ & CDCP & Natl Institute for Occupational Safety \& Heal & & $000000 \mathrm{R} 1 \mathrm{OH} 03078$ & Extramun \\
\hline 471 & HHS & CDCP & Nall Institute for Occupational Safoty \& Heal & & $000000 R 10+103154$ & Extramun \\
\hline 472 & HHS & CDCP & Natl Institute for Occupational Safety \& Heal & & $000000 \mathrm{R} 3 \mathrm{OH} 02931$ & Extramun \\
\hline 473 & HHS & CDCP & Natl Institute for Occupational Safety \& Heal & & $000000 \mathrm{R} 3 \mathrm{OH} 02938$ & Extramur \\
\hline 474 & $\mathrm{HHS}$ & CDCP & Nafl institute for Occupational Safety \& Heal & & $000000 \mathrm{R} 3 \mathrm{OH} 02966$ & Extramun \\
\hline 475 & HHS & CDCP & Natl Institute for Occupational Safety \& Heal & & $000000 \mathrm{R} 30 \mathrm{H} 02972$ & Extramur \\
\hline 476 & HHS & CDCP & Natl Institute for Occupational Safety \& Heal & & $000000 \mathrm{R} 3 \mathrm{OH} 03000$ & Extramun \\
\hline 477 & HHS & CDCP & Nafl Institute for Occupational Safety \& Heal & & $000000 R 30 \mathrm{H} 03039$ & Extramur \\
\hline 478 & HHS & CDCP & Natl Institute for Occupational Safety \& Heal & & $000000 \mathrm{R} 30 \mathrm{H} 03061$ & Extramut \\
\hline 479 & HHS & CDCP & Natl Institute for Occupational Safety \& Heal & & $000000 R 30 H 03099$ & Extramut \\
\hline 480 & HHS & CDCP & Natl Institute for Occupational Safiety \& Heal & & $000000 \mathrm{R} 30 \mathrm{H} 03102$ & Extramur \\
\hline 481 & HHS & CDCP & Natl Institute for Occupational Safety \& Heal & & $000000 R 30 \mathrm{H} 03103$ & Extramur \\
\hline 482 & HHS & CDCP & Natl Institute for Occupational Safety \& Heal & & $000007 \mathrm{~K} 1 \mathrm{OH} 00106$ & Extramur \\
\hline 483 & $\mathrm{HHS}$ & NIH & National Center for Research Resources & Comparative medicine (extramural, includes trai & G2ORR0 $8 \pi 77$ & $E / G$ \\
\hline 484 & HHS & $\mathrm{NIH}$ & National Center for Research Resources & Comparative medicine (extramural, includes trai & G20RR08792 & $E / G$ \\
\hline 485 & HHS & $\mathrm{NIH}$ & National Institute of Environmental Health S & Supertund research program - funds transferrec & P42ES04699 & $E / G$ \\
\hline 486 & HHS & $\mathrm{NIH}$ & National Institute of Environmental Health S & [Supertund research program - funds transferreo & P42ESO4917 & $E / G$ \\
\hline 487 & HHS & $\mathrm{NIH}$ & National Institute of Environmental Health S & [Superfund research program - funds transferreo & P42ES04940 & $E / G$ \\
\hline 488 & HHS & NIH & National Institute of Environmental Health S & Superfund research program - funds transferrec & P42ES05946 & $E / G$ \\
\hline 489 & HHS & NIH & National Institute of Environmental Health S & Superfund research program - funds transferreo & P42ES05949 & $E / G$ \\
\hline 490 & HHS & NIH & National Institute of Environmental Health S & Biometry \& nisk estimation (extramural, includes & RotES05410 & $E / G$ \\
\hline 491 & HHS & $\mathrm{NIH}$ & National Institute on Aging & Aging (exramural, includes training) & R43AG12343 & $E / G$ \\
\hline 492 & HHS & $\mathrm{NIH}$ & National Institute of Environmental Health S & Applied toxicological research \& testing (extram & R43ESO5898 & $E / G$ \\
\hline 493 & HHS & $\mathrm{NIH}$ & National Institute on Aging & Aging (extramural, includes training) & R44AG08903 & $E / G$ \\
\hline 494 & HHS & NIH & National Institute of Environmental Health S & Applied toxicological research \& testing (extram & U45ES 06167 & $E / G$ \\
\hline 495 & NASA & SAT & Small Business Innovative Research (SBIR) & & NAS13603 & $E / C$ \\
\hline 496 & NSF & ENG & Civil \& mechanical systems (CMS) & Structural Systems \& Construction Processes & 8657142 & $E / G$ \\
\hline 497 & NSF & ENG & Civil \& mechanical systems (CMS) & Large Structural \& Building Systems & 8657642 & $E / G$ \\
\hline 498 & NSF & ENG & Civil \& mechanical systems (CMS) & Geomochanical/Geotech \& Geo-Environmental & 8657999 & $E / G$ \\
\hline 499 & NSF & ENG & Civil \& mechanical systems (CMS) & Structural Systems \& Construction Processes & 8658102 & $E / G$ \\
\hline 500 & NSF & ENG & Civil \& mechanical systems (CMS) & Natural \& Man-Made Hazard Mitigation & 8821163 & $E / C A$ \\
\hline 501 & NSF & ENG & Civil \& mechanical systems (CMS) & Natural \& Man-Made Hazard Mitigation & 8821542 & $E / C A$ \\
\hline 502 & NSF & ENG & Civil \& mechanical sysiems (CMS) & Structural Systems & 8857002 & $E / G$ \\
\hline 503 & NSF & ENG & Civil \& mochanical systems (CMS) & Architectural \& Mechanical Systems & 8857080 & $E / G$ \\
\hline 504 & NSF & ENG & Bioengineering \& environmental systems (B) & Bioengineering \& environmental systems - Othe & 8857500 & $E / G$ \\
\hline 505 & NSF & ENG & Civil \& mechanical systems (CMS) & Stuctural Systems \& Construction Processes & 8857749 & $E / G$ \\
\hline 500 & NSF & ENG & Civil \& mechanical systems (CMS) & Structural Systems & 8858549 & $E / G$ \\
\hline 507 & NSF & ENG & Civil \& mechanical systems (CMS) & Siling \& Geotechnical Systems & 8912074 & $E / G$ \\
\hline 500 & NSF & ENG & Civil \& mechanical systems (CMIS) & Large Structural \& Building Systems & 8913129 & $E / G$ \\
\hline 509 & NSF & ENG & Civil \& mechanical systems (CMS) & Large Structural \& Building Systems & 8914008 & $E / G$ \\
\hline 510 & NSF & ENG & Civil \& mechanical systems (CMS) & Structural Systems \& Constnuction Processes & 8921586 & $E / G$ \\
\hline 511 & NSF & ENG & Civil \& mechanical systems (CMS) & Siting \& Geotechnical Systems & 8922867 & $E / G$ \\
\hline 512 & NSF & ENG & Civil \& mechanical systems (CASS) & Siting \& Geotechnical Systems & 8922869 & $E / G$ \\
\hline 513 & NSF & ENG & Engineering education \& centers (EEC) & Engineering education \& centers - Other & 8943455 & ESCA \\
\hline 514 & NSF & ENG & Civil \& mechanical systems (CMS) & Struciural Systems \& Construction Processes & 8957131 & $E / G$ \\
\hline 515 & NSF & ENG & Civil \& mechanical systems (CMS) & Large Structural \& Building Systems & 8957183 & $E / G$ \\
\hline 516 & NSF & ENG & Civil \& mechanical systems (CMS) & Earthquake Systems Integration & 8957425 & $E / G$ \\
\hline 517 & INSF & ENG & Design, manufacture \& industrial innovation & Engineering Design & 8957464 & $E / G$ \\
\hline 518 & NSF & ENG & Civil \& mechanical systems (CMS) & Large Structural \& Building Systems & 8957853 & $E / G$ \\
\hline 519 & NSF & ENG & Civil \& mechanical systems (CMS) & structural Systems \& Construction Processes & 8958316 & $E / G$ \\
\hline 520 & NSF & ENG & Civil \& mechanical systems (CMS) & Siting \& Geotechnical Systems & 8958402 & $E / G$ \\
\hline
\end{tabular}




\begin{tabular}{|c|c|c|c|c|c|c|c|c|c|c|c|c|c|}
\hline & Agency & $\begin{array}{l}\text { Start } \\
\text { Date }\end{array}$ & $\begin{array}{l}\text { End } \\
\text { Date }\end{array}$ & $\begin{array}{c}\text { Place of } \\
\text { Performance }\end{array}$ & $\begin{array}{l}\text { : } \\
\text { के } \\
: 0 \\
0\end{array}$ & Performer Name & $\begin{array}{l}\text { Pert }=\frac{\bar{\Sigma}}{\Phi} \\
\text { Type }\end{array}$ & $\begin{array}{c}\text { Total } \\
\text { Award } \\
\text { Amount }\end{array}$ & $\begin{array}{c}\text { Average } \\
\text { Annual } \\
\text { Funding }\end{array}$ & $\begin{array}{l}\text { FY } \\
\text { Total } \\
\text { Amt }\end{array}$ & $\begin{array}{c}\text { FY Fod } \\
\text { Amt }\end{array}$ & $\begin{array}{c}\text { FY } \\
\text { Non- } \\
\text { Fed } \\
\text { Amt }\end{array}$ & $\begin{array}{c}\text { Prod. } \\
*\end{array}$ \\
\hline 456 & HHS & Sep-92 & Sep-94 & New York & NY & CUNY, RESEARCH FOUND & StalocGov & & 112.483 & & & & 7 \\
\hline 457 & HHS & Feb-92 & $\operatorname{Jan}-94$ & New Orleans & LA & TULANE UNIVERSITY OF L & PriEdinst & & 104.868 & & & & 7 \\
\hline 458 & HHS & Sep-93 & Aug-94 & Lowell & MA & UNIVERSITY OF LOWELL & Edlnst & & 48.493 & & & & 7 \\
\hline 459 & HHS & May-92 & Apr-94 & Now York & NY & CORNELL UNIVERSITY, MI & PriEdlnst & & 147.892 & & & & 7 \\
\hline 460 & HHS & May-92 & Apr-94 & Boston & MA & BOSTON UNIVERSITY & PriEdlnst & & 181.294 & & & & 7 \\
\hline 461 & HHS & Sep-92 & Aug-94 & Rochester & NY & UNIVERSITY OF ROCHES & PriEdlnst & & 185.286 & & & & 7 \\
\hline 462 & HHS & od-91 & Nov-93 & Chicago & IL & UNIVERSITY OFILLINOIS & Edlnst & & 52.832 & & & & 7 \\
\hline 463 & HHS & Apr-92 & \begin{tabular}{|l|} 
Jun-94 \\
\end{tabular} & Chapel Hill & $\mathrm{NC}$ & UNIVERSITY OF NORTHC & Edinst & & 61.022 & & & & 7 \\
\hline 464 & HHS & Sep-92 & Sep-94 & Los Angeles & $\mathrm{CA}$ & UNIVERSITY OF CALIFORI & EdInst & & 187.726 & & & & 7 \\
\hline 465 & HHS & Aug-92 & Jul-94 & Now York & NY & COLUMBIA UNIVERSITY, \$ & PriEdlnst & & 252.346 & & & & 7 \\
\hline 466 & HHS & Sep-92: & Sep-94 & Albuquerque & NM & LOVELACE BIOMEDICAL \& & NonproinonE & & 178.573 & & & & 7 \\
\hline 467 & $\mathrm{HHS}$ & Apr-92 & Aug-94 & Cincinnati & OAH & UNIVERSITY OF CINCINNA & Edlnst & & 143.525 & & & & 7 \\
\hline 468 & HHS & May-92 & Apr-94 & Ann Abor & MI & UNIVERSITY OF MICHIGAN & EdInst & & 94.533 & & & & 7 \\
\hline 469 & HHS & Sep-93 & Sep-94 & State College & PA & PENNSYLVANIA STATE UI & Edinst & & 114.33 & & & & 7 \\
\hline 470 & HHS & Sep-93 & Sep-94 & Atlanta & GA & EMORY UNIVERSITY & PriEdlnst & & 136.688 & & & & 7 \\
\hline 471 & HHS & Sep-93 & Sep-94 & Ann Arbor & $\mathrm{MI}$ & UNIVERSITY OF MICHIGAS & Edlnst & & 36.92 & & & & 7 \\
\hline 472 & HHS & Oct-91 & Dec-93 & New York & NY & COLUMBIA UNIVERSITY, $\$$ & PriEdlnst & & 23.885 & & & & 7 \\
\hline 473 & HHS & Dec-92 & Nov-93 & Minneapolis & MN & UNIVERSITY OF MINNESO & Edlnst & & 12.814 & & & & 7 \\
\hline 474 & HHS & Jan-92 & Mar-94 & Seattle & WA & UNIVERSITY OF WASHINC & EdInst & & 22.604 & & & & 7 \\
\hline 475 & HHS & Aug-92 & Jul-94 & Cincinnati & $\mathrm{OH}$ & UNIVERSITY OF CINCINNA & Edinst & & 11.965 & & & & 7 \\
\hline 476 & HHS & Sep-92 & Sep-94 & Davis & $\mathrm{CA}$ & UNIVERSITY OF CALIFORI & Edinst & & 17.835 & & & & 7 \\
\hline 477 & $\mathrm{HHS}$ & Sep-92 & Sep-94 & New York & NY & NEW YORK UNIVERSITYN & PriEdINEW Y & TORK UN & 25.298 & & & & 7 \\
\hline 478 & HHS & Jun-93 & May-94 & Little Rock & AR & UNIVERSITY OF ARKANSA & StalocGov & & 34.64 & & & & 7 \\
\hline 479 & HHS & Sep-93 & Sep-94 & Seattle & WA & MEDICAL CONSULTANTS & IndBus & & 49.225 & & & & 7 \\
\hline 480 & HHS & Sep-93 & Mar-94 & Berkeley & CA & BERKELEY MICROINSTRU & IndBus & & 49.975 & & & & 7 \\
\hline 481 & HHS & Sep-93 & Mar-94 & Springfield & VA & MICROSENSOR SYSTEMS & IndBus & & 49.616 & & & & 7 \\
\hline 482 & HHS & Aug-92 & Jun-94 & Cambridge & MA & HARVARD UNIVERSITY PF & PriEdinst & & 54 & & & & 7 \\
\hline 483 & HHS & Sep-93 & Aug-94 & Detroit & MI & WAYNE STATE UNIVERSI & Edlinst & & 470.3 & & & & $\overline{7}$ \\
\hline 484 & HHS & Sep-93 & Sep-94 & New York & NY & CORNELL UNIVERSITY, MI & PriEdlnst & & 612.659 & & & & 7 \\
\hline 485 & HHS & Jan-92 & Mar-96 & Davis & CA & UNIVERSITY OF CALIFORI & Edlnst & & 1429.066 & 1762.2 & 1762.2 & 0 & 7 \\
\hline 486 & HHS & Apr-92 & Mar-96 & College Station & $T X$ & TEXAS A \& M UNIVERSITY & StalocGov & & 668.516 & 730.9 & 730.9 & 0 & 7 \\
\hline 487 & HHS & Apr-92 & Mar-95 & Tucson & $A Z$ & UNIVERSITY OF ARIZONA & Edlnst & & 1816.005 & 1830.5 & 1830.5 & o & 7 \\
\hline 488 & HHS & Apr-92 & Mar-95 & New Orleans & LA & TULANE UNIVERSITY OF & PriEdinst & & 1764.906 & 1492.3 & 1492.3 & 0 & 7 \\
\hline 489 & HHS & Apr-92 & Mar-96 & Font Collins & $\mathrm{CO}$ & COLORADO STATE UNIVE & Edlinst & & 783.071 & 877.21 & 877.21 & 0 & 7 \\
\hline 490 & HHS & Sep-92 & Aug-95 & New Haven & CT & JOHN B PIERCE FOUNDAT & NonprofiNonE & & 734.639 & 829.13 & 829.13 & 0 & 7 \\
\hline 491 & HHS & May-94 & Nov-94 & Wellesiey (Tow & NMA & HEARTHSTONE AL ZHEIME & IndBus & & 53.5 & 53.5 & 53.5 & 0 & 5 \\
\hline 492 & HHS & Jun-94 & Nov-94 & Bedford (Town & IMA & NITON CORPORATION & IndBus & & 67.192 & 67.192 & 67.192 & 0 & 7 \\
\hline 493 & HHS & Feb-92 & Jan-94 & Bowie & $M D$ & BONNIE WALKER \& ASSO & IndBus & & 250 & & & & 8 \\
\hline 494 & HHS & Sep-93 & Aug-94 & Washington & $D C$ & INTERNATIONAL ASSOCIA & Oth & & 135 & & & & 5 \\
\hline 495 & NASA & Jan-94 & Jul-94 & Auburn & CA & NO PERFORMER INFO AV & NotSpec & 69.95 & 70 & 70 & 70 & & 7 \\
\hline 490 & NSF & Jul-87 & Aug-94 & West Lafayette & IN & PURDUE UNIVERSITY & EdInst & 312 & 37.5 & & & & 8 \\
\hline 497 & NSF & Jul-87 & Dec-93 & Seattle & WA & UOF WASHINGTON & Edlnst & 231.038 & 34.845 & & & & 7 \\
\hline 498 & NSF & Sep-87 & 0at-94 & Los Angeles & $C A$ & UOF SOUTHERN CALIFOF & PriEdinst & 271.6 & 34.1 & & & & 7 \\
\hline 499 & NSF & Jul-87 & Dec-94 & Berketey & CA & U OF CAL BERKELEY & Edinst & 312.5 & 62.5 & & & & 7 \\
\hline 500 & NSF & Jun-89 & May-95 & Lubbock & $T X$ & TEXAS TECH UNIVERSITY & Edinst & 1270.5 & 280.308 & 25 & 25 & 0 & 7 \\
\hline 501 & NSF & Jun-89 & May-95 & Fort Collins & $\mathrm{CO}$ & COLORADO STATE UNIVE & Edlnst & 1264.84 & 280.297 & 25 & 25 & 0 & 8 \\
\hline 502 & NSF & Sep-88 & Feb-94 & Ann Arbor & $\mathrm{MI}$ & NO PERFORMER INFO AV & NotSpec & 129.5 & 25 & & & & 7 \\
\hline 503 & NSF & Sep-88 & Feb-94 & Bưffalo & NY & SUNY BUFFALO & Edlnst & 312 & 62.5 & & & & 7 \\
\hline 504 & NSF & Sep-88 & Aug-94 & Ann Arbor & MI & NO PERFORMER INFO AV & NotSpoc & 304.898 & 62.5 & & & & 7 \\
\hline 505 & NSF & Od-88 & May-95 & Cambridge & MA & MIT & PriEdinst & 250 & 62.5 & 62.5 & 62.5 & 0 & 7 \\
\hline 506 & NSF & Sep-88 & Feb-94 & Evanston & IL & NORTHWESTERN UNIVEA & PriEdlnst & 274.5 & 50 & & & & 7 \\
\hline 507 & NSF & Oct-89 & Sep-94 & Davis & $\overline{C A}$ & U OF CAL DAVIS & Edlnst & 543.549 & 217.601 & 88.201 & 88.201 & 0 & 7 \\
\hline 508 & NSF & Mar-90 & Nov-94 & Worcester & MA & WORCESTER POLYTECH & PriEdlnst & 77.025 & 23.106 & & & & 7 \\
\hline 509 & NSF & Jun-90 & Nov-94 & Boulder & $\mathrm{CO}$ & U OF COLORADO BOULDE & Edlnst & 242.236 & 81.361 & & & & 7 \\
\hline 510 & NSF & Nov-90 & Oct-94 & Madison & WI & U OF WISCONSIN MADISO & Edlnst & 150 & 80 & & & & 7 \\
\hline 511 & NSF & Nov-89 & Apr-94 & Gainesville & $\mathrm{FL}$ & U OF FLORIDA & Edlnst & 102.389 & 27.367 & & & & 7 \\
\hline 512 & NSF & Feb-90 & Jul-94 & Princelon & NS & PRINCETON UNIVERSITY & PriEdlnst & 188.573 & 40.398 & & & & 7 \\
\hline 513 & NSF & May-89 & Apr-98 & Bethlehem & PA & LEKIGH UNIVERSITY & PriEdinst & 17975.1 & 3497.38 & 2750 & 2750 & 0 & 7 \\
\hline 514 & NSF & Jul-89 & Jun-94 & thaca & NY & CORNELL UNIVERSITY-EN & PriEdinst & 250 & 41.667 & 22.5 & 22.5 & 0 & 7 \\
\hline 515 & NSF & Jul-89 & Jun-95 & Berkeley & $\overline{C A}$ & U OF CAL BERKELEY & Edlnst & 212 & 62.5 & 62.5 & 62.5 & 0 & 7 \\
\hline 516 & NSF & Aug-89 & Jul-95 & Los Angeles & $\overline{C A}$ & U OF SOUTHERN CALIFOF & PriEdinst & 146.5 & 47.5 & & & & 7 \\
\hline 517 & NSF & Sep-89 & Jan-95 & Cambridge & MA & MIT & PriEdlnst & 249.5 & 62.5 & & & & 6 \\
\hline 518 & NSF & Aug-89 & $\operatorname{Jan}-95$ & Los Angeles & $\mathrm{CA}$ & U OF SOUTHERN CALIFOF & PriEdinst & 221.559 & 67.03 & & & & 7 \\
\hline 519 & NSF & $0 d-89$ & Mar-95 & Palo Allo & $C A$ & STANFORD UNIVERSITY & ¡PriEdinst & 244 & 62.5 & & 25 & 0 & 6 \\
\hline 520 & $\overline{N S} \bar{F}$ & Jul-89 & Dec-94 & Boulder & $\mathrm{CO}$ & U OF COLORADO BOULDE & Edlnst & 212 & 57.5 & 37.5 & 37.5 & 0 & 7 \\
\hline
\end{tabular}




\begin{tabular}{|c|c|c|c|c|c|c|}
\hline & Agency & Bureau & Program & Project & Award Number & $\begin{array}{c}\text { Award } \\
\text { Type }\end{array}$ \\
\hline 521 & NSF & ENG & Civil \& mechanical systems (CMS) & Siting \& Goolechnical Systems & 9000378 & $E / G$ \\
\hline 522 & NSF & ENG & Civil \& mechanical systems (CMS) & Siting \& Geolechnical Systems & 9000379 & $E / G$ \\
\hline 523 & NSF & ENG & Civil \& mechanical systems (CMS) & Siting \& Geolechnical Systems & 9000390 & $E / G$ \\
\hline 524 & NSF & ENG & Civil \& mechanical systems (CMS) & Sructural Systems & 9001494 & $E / G$ \\
\hline 525 & NSF & ENG & Civil \& mechanical systems (CMS) & Siructural Systems \& Construction Processes & 9002513 & $E / G$ \\
\hline 526 & NSF & ENG & Civil \& mectranical systems (CMS) & Sinuctural Systems \& Consiruction Processes & 9002571 & E/G \\
\hline 527 & NSF & ENG & Civil \& mechanical systems (CMS) & Sinctural Systems & 9006781 & $E / G$ \\
\hline 528 & NSF & ENG & Civil \& mechanical systems (CMS) & Large Structural \& Building Systems & 9011065 & $E / G$ \\
\hline 529 & NSF & ENG & Civil \& mechanical systems (CMS) & Architectural \& Mochanical Systems & 9011671 & E/G \\
\hline 530 & NSF & ENG & Civil \& mochanical systems (CMS) & Architectural \& Mechanical Systems & 9011672 & $E / G$ \\
\hline 531 & NSF & ENG & Civil \& mochanical systems (CMS) & Architectural \& Mechanical Systems & 9011674 & $E / G$ \\
\hline 532 & NSF & ENG & Civil \& mechanical systems (CMS) & Architectural \& Mechanical Systems & 9011676 & E/G \\
\hline 533 & NSF & ENG & Civil \& mechanical systems (CMS) & Earthquake Systems integralion & 9013334 & $E / G$ \\
\hline 534 & NSF & ENG & Civil \& mochanical systems (CMS) & Large Structural \& Building Systems & 9014808 & $E / G$ \\
\hline 535 & NSF & ENG & Civil \& mechanical systems (CMS) & Natural \& Man-Made Hazard Mitigation & 9015272 & $E / G$ \\
\hline 536 & NSF & ENG & Civil \& mechanical systems (CMS) & Structural Systems & 9015291 & E/G \\
\hline 537 & NSF & CSE & Information, robotics \& intelligent systems & Information Tochnology \& Organizations & 9015441 & $E / G$ \\
\hline 538 & NSF & ENG & Civil \& mechanical systems (CMS) & Structural Systems \& Construction Processes & 9015502 & $E / G$ \\
\hline 539 & NSF & ENG & Civil \& mechanical systems (CMS) & Large Siructural \& Building Systems & 9015542 & $E / G$ \\
\hline 540 & NSF & ENG & Civil \& mechanical systems (CMS) & Natural \& Man-Made Hazard Mitigation & 9015850 & $E / G$ \\
\hline 541 & NSF & ENG & Civil \& mechanical systems (CMS) & STructural Systems \& Construction Procosses & 9015912 & $E / G$ \\
\hline 542 & NSF & ENG & Civil \& mechanical systems (CMS) & STructural Systems \& Construction Procosses & 9015978 & $E / G$ \\
\hline 543 & NSF & ENG & Civil \& mochanical systems (CMS) & Large Structural \& Building Systems & 9016459 & $E / G$ \\
\hline 544 & NSF & ENG & Civil \& mechanical systems (CMS) & Structural Systems & 9016509 & $E / G$ \\
\hline 545 & NSF & ENG & Civil \& mochanical systems (CMS) & Structural Systems & 9016714 & E/G \\
\hline 546 & NSF & ENG & Civil \& mechanical systems (CMS) & Structural Systems & 9016828 & $E / G$ \\
\hline 547 & NSF & ENG & Civil \& mechanical systems (CMS) & Structural Systems & 9016863 & $E / G$ \\
\hline 548 & NSF & ENG & Civil \& mechanical systems (CMS) & Structural Systems & 9016867 & E/G \\
\hline 549 & NSF & ENG & Civil \& mechanical systems (CMS) & Structural Systems & 9016873 & $E / G$ \\
\hline 550 & NSF & ENG & Civil \& mechanical systems (CMS) & Siructural Systems & 9016875 & $E / G$ \\
\hline 551 & NSF & ENG & Civil \& mechanical systems (CMS) & Siting \& Geolechnical Systems & 9016880 & $E / G$ \\
\hline 552 & NSF & ENG & \begin{tabular}{|l} 
Civil \& mechanical systems (CMS) \\
\end{tabular} & Large Structural \& Building Systems & 9017149 & $E / G$ \\
\hline 553 & NSF & ENG & Civil \& mechanical systems (CMS) & Large Structural \& Building Systems & 9017771 & $E / G$ \\
\hline 554 & NSF & ENG & Civil \& mechanical systems (CMS) & Large Structural \& Building Systems & 9018519 & $E / G$ \\
\hline 555 & NSF & ENG & Civil \& mechanical systems (CMS) & Structural Systems \& Construction Processes & 9018572 & $E / G$ \\
\hline 556 & NSF & ENG & Civil \& mechanical systems (CMS) & Structural Systems \& Construction Processes & 9018572 & $E / G$ \\
\hline 557 & NSF & ENG & Civil \& mechanical systems (CMS) & Structural Systems \& Construction Processes & 9018572 & $E / G$ \\
\hline 558 & NSF & ENG & Civil \& mechanical systems (CMS) & Structural Systems \& Construction Processes & 9021066 & $E / G$ \\
\hline 559 & NSF & ENG & Civil \& mechanical systems (CMS) & Structural Systems \& Construction Processes & 9021995 & $E / G$ \\
\hline 560 & NSF & ENG & Civil \& mechanical systems (CMS) & Large Strucural \& Building Systems & 9022667 & $E / G$ \\
\hline 561 & NSF & ENG & Civil \& mechanical systems (CMS) & Natural \& Man-Made Hazard Mitigation & 9022714 & $E / G$ \\
\hline 562 & NSF & ENG & Chemical \& transport systems (CTS) & Process \& Reaction Engineering & 9023240 & E/G \\
\hline 563 & NSF & ENG & Civil \& mechanical systems (CMS) & Large Structural \& Building Systems & 9024976 & $E / G$ \\
\hline 564 & NSF & ENG & Civil \& mechanical systems (CMS) & Structural Systems & 9057052 & $E / G$ \\
\hline 565 & NSF & ENG & Bioengineering \& environmental systems (E & Environmental Engineering Systems & 9057298 & $E / G$ \\
\hline 566 & NSF & ENG & Chemical \& transport systems (CTS) & Combustion \& Thermal Plasmas & 9057963 & $E / G$ \\
\hline 567 & NSF & ENG & Civil \& mechanical systems (CMS) & Structural Systems \& Construction Procosses & 9058005 & $E / G$ \\
\hline 568 & NSF & ENG & Civil \& mechanical systems (CMS) & Large Structural \& Building Systems & 9058092 & $E / G$ \\
\hline 569 & NSF & ENG & \begin{tabular}{|l|} 
Civil \& mechanical systems (CMS) \\
\end{tabular} & Large Structural \& Building Systems & 9058146 & $E / G$ \\
\hline 570 & NSF & ENG & Civil \& mechanical systems (CMS) & Structural Systems \& Construction Procosses & 9058304 & $E / G$ \\
\hline 571 & NSF & ENG & Civil \& mechanical systems (CMS) & Struclural Systems & 9058316 & $E / G$ \\
\hline 572 & NSF & ENG & Civil \& mechanical systems (CMS) & Structural Systems \& Construction Processes & 9058545 & $E / G$ \\
\hline 573 & NSF & ENG & Civil \& mechanical systems (CMS) & Structural Systems \& Construction Processes & 9096274 & $E / G$ \\
\hline 574 & NSF & ENG & Civil \& mechanical systems (CMS) & Large Structural \& Building Systems & 9100627 & $E / G$ \\
\hline 575 & NSF & ENG & Design, manufacture \& industrial innovation & SBIR-Phase II & 9101585 & $E / G$ \\
\hline 576 & NSF & ENG & Civil \& mechanical systems (CMS) & Struclural Systems & 9102430 & $E / G$ \\
\hline 577 & NSF & ENG & Design, manufacture \& industrial innovalion & Production Systems & 9102993 & $E / G$ \\
\hline 578 & NSF & ENG & Civil \& mechanical systems (CMS) & Large Structural \& Building Systems & 9105072 & $E / G$ \\
\hline 579 & NSF & ENG & Civil \& mechanical systems (CMS) & Large Structural \& Building Systems & 9105194 & $E / G$ \\
\hline 580 & NSF & ENG & Engineening education \& centers (EEC) & Engineering education \& centers - Other & 9106202 & $E / G$ \\
\hline 581 & NSF & ENG & Civil \& mochanical systems (CMS) & Architeclural \& Mechanical Systems & 9106390 & $E / G$ \\
\hline 582 & NSF & SBE & International cooperative scientific activities & Intemational activities - Other & 9106732 & $E / G$ \\
\hline 583 & NSF & ENG & Civil \& mechanical systems (CMS) & Large Structural \& Building systems & 9108064 & $E / G$ \\
\hline 584 & NSF & EHR & Systemic reformVEPSCOR (OSR) & & 9108774 & $E / G$ \\
\hline 585 & NSF & ENG & Civil \& mochanical systems (CMS) & Large Structural \& Building Systems & 19109024 & $E / G$ \\
\hline
\end{tabular}




\begin{tabular}{|c|c|c|c|c|c|c|c|c|c|c|c|c|c|}
\hline & Agency & $\begin{array}{l}\text { Start } \\
\text { Date }\end{array}$ & $\begin{array}{l}\text { End } \\
\text { Date }\end{array}$ & $\begin{array}{c}\text { Place of } \\
\text { Performance }\end{array}$ & 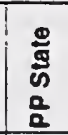 & Performer Name & $\begin{array}{c}\text { Perf } \\
\text { Typo }\end{array}$ & $\begin{array}{c}\text { Total } \\
\text { Award } \\
\text { Amount }\end{array}$ & $\begin{array}{c}\text { Average } \\
\text { Annual } \\
\text { Funding }\end{array}$ & $\begin{array}{c}\text { FY } \\
\text { Total } \\
\text { Amt }\end{array}$ & $\begin{array}{c}\text { FY Fod } \\
\text { Amt }\end{array}$ & \begin{tabular}{|c|} 
FY \\
Non- \\
Fod \\
Amt \\
\end{tabular} & Prod. \\
\hline 521 & NSF & Dec-89 & Nov-93 & Cambridge & MA & MIT & Priedinst & 223.376 & 39.872 & & & & 7 \\
\hline 522 & NSF & $D 00-89$ & Nov-94 & Boulder & $\mathrm{CO}$ & U OF COLORADO BOULDE & Edinst & \begin{tabular}{|l|}
235.887 \\
\end{tabular} & 64.677 & & & & 7 \\
\hline 523 & NSF & Dec-89 & Apt-94 & Pasadena & $\mathrm{CA}$ & CALIFORNIA INST OF TEC & PriEdinst & 255.573 & 80.973 & & & & 7 \\
\hline 524 & NSF & Apr-90 & Mar-94 & Rolla & MO & U OF MISSOURI ROLLA & Edlnst & 214.999 & 5.25 & & & & 7 \\
\hline 525 & NSF & Nov-90 & Oct-93 & Urbana & IL & U OF ILLINOIS URBANA & Eding & 128.5 & 52.432 & & & & 7 \\
\hline 526 & NSF & Dec-90 & Nov-93 & Mitwaukee & WI & MAROUETTE UNIVERSITY & Priedinst & 107.626 & 5 & & & & 7 \\
\hline 527 & NSF & Nov-90 & Oat-93 & Norre Dame & IN & UNIVERSITY OF NOTRE D & PriEdlnst & 100.169 & 10 & & & & 7 \\
\hline 528 & NSF & Jun-90 & Nov-94 & Boulder & Co & ATKINSON-NOLAND ASSC & IndBus & 171.443 & 58.479 & & & & 7 \\
\hline 529 & NSF & May-90 & Apr-94 & Los Angeles & CA & NO PERFORMER INFO AV & NotSpec & 100 & 20 & & & & 7 \\
\hline 530 & NSF & May-90 & Oat-93 & Los Angeles & CA & UOF CAL LOS ANGELES & Edinst & 158.477 & 59.999 & & & & 7 \\
\hline 531 & NSF & May -90 & Odt-94 & Berkeley & $\mathrm{CA}$ & UOF CAL BERKELEY & Edinst & 227 & 90 & & & & 7 \\
\hline 532 & NSSF- & May-90 & Oci-93 & Seattle & WA & UOF WASHINGTON & Edlnst & 147 & 30 & & & & 7 \\
\hline 533 & NSF & Jan-91 & Dec-94 & Berkeley & CA & UOF CAL BERKELEY & Edlnst & 1380.66 & 470.166 & & & & 3 \\
\hline 534 & NSF & Aug-91 & Jan-96 & Southfield & MI & LAWRENCE TECHNICALC & PriEdlnst & 117.02 & 22.617 & 8.9 & 8.9 & 0 & 7 \\
\hline 535 & NSF & Jan-91 & Dec-94 & Boca Raton & $F$ & FLORIDA ATLANTIC UNIV & Edlnst & 271.881 & 177.635 & & & & 7 \\
\hline 536 & NSF & Jun-90 & May-95 & Berkeley & $\mathrm{CA}$ & UOF CAL BERKELEY & Edinst & 574.143 & 50 & & & & 7 \\
\hline 537 & NSF & Sep-90 & Aug-94 & Boulder & Co & UOF COLORADO BOULDE & Edlnst & 745 & 290 & & & & 6 \\
\hline 538 & NSF & Dec-90 & Nov-93 & Washington & $D C$ & CATHOLIC UNIVERSITY & PriEdinst & 120.238 & 42 & & & & 7 \\
\hline 539 & NSF & Jun-91 & May-94 & Cambridge & $\overline{M A}$ & MIT & PriEdlns & 100 & 50 & & & & 7 \\
\hline 540 & NSF & $\operatorname{Jan}-91$ & Dec-94 & Lubbock & $\overline{T X}$ & TEXAS TECH UNIVERSITY & E Elinst & 246.882 & 27.965 & 3.186 & $3 . \overline{186}$ & 0 & 7 \\
\hline 541 & NSF & May-91 & Apr-94 & Knoxville & $T N$ & UOF TENNESSEE KNOXV & Edinst & 110 & 110 & & & & 7 \\
\hline 542 & NSF & Jun-91 & Nov-93 & Evanston & IL & NORTHWESTERN UNIVEF & PriEdlnst & 100.717 & 52.534 & & & & 7 \\
\hline 543 & NSF & Aug-91 & Jan-95 & Morgantown & WV & WV UNIV RESEARCH COR & Edlnst & 210.313 & 110.48 & 114.88 & 114.88 & 0 & 7 \\
\hline 544 & NSF & Sep-90 & Feb-94 & Urbana & IL & U OF ILLINOIS URBANA & Edinst & 191.999 & 64 & & & & 7 \\
\hline 545 & NSF & Oct-90 & Sep-94 & Seattle & WA & UOF WASHINGTON & Edinst & 300 & 120 & & & & 7 \\
\hline 546 & NSF & Aug-90 & \begin{tabular}{|l|} 
Jan-94 \\
\end{tabular} & Austin & $T X$ & U OF TEXAS AUSTIN & Edlnst & 243.3 & 58.65 & & & & 7 \\
\hline 547 & NSF & Sep-90 & Aug-94 & Ann Arbor & MI & U OF MICHIGAN ANN ARB & EdInst & 290.491 & 28.545 & 13.789 & 13.789 & $\overline{0}$ & 7 \\
\hline 548 & $\bar{N} S F$ & 0 ct- -90 & Jun-94 & Los Angeles & $C A$ & ENGLEKIRK\&SABOL CON & indBus & 16.945 & 11.896 & & & & 7 \\
\hline 549 & NSF & Sep-90 & Feb-95 & San Francisco & $C A$ & HJ DEGENKOLB ASSOCS & IndBus & 34.722 & 11.74 & & & & 7 \\
\hline 550 & NSF & Sep-90 & Feb-94 & San Francisco & $C A$ & SOH ASSOCIATES & IndBus & 48 & 16 & & & & 7 \\
\hline 551 & NSF & Aug-90 & Jul-94 & Troy & NY & RENSSELAER POLYTECH & PriEdInst & 274.449 & 54.388 & & & & 7 \\
\hline 552 & NSF & Jun-91 & May-95 & Boulder & Co & UOF COLORADO BOULDE & Edlnst & 128.862 & 44.999 & 67.663 & 67.663 & 0 & 7 \\
\hline 553 & NSF & Mar-91 & Feb-94 & thaca & NY & CORNELL UNIVERSITY EA & PriEdinst & 272.974 & 147.002 & & & & 7 \\
\hline 554 & NSF & Feb-91 & Dec-93 & Mayaguez & PR & U OF PUERTO RICO MAYA & Edlnst & 170.258 & 26.559 & & & & 7 \\
\hline 555 & NSF & Noy -90 & Mar-94 & Piscataway (To & $N$ & NO PERFORMER INFO AV & NotSpec & 207.818 & 92.023 & & & & 7 \\
\hline 556 & NSF & Nov-90 & Mar-94 & Piscataway (To & NN & NO PERFORMER INFO AV & NotSpec & 207.818 & 92.023 & & & & 7 \\
\hline 557 & NSF & Nov-90 & Mar-94 & Piscataway (To & NW & NO PERFORMER INFO AV & NoISpec & 207.818 & 92.023 & & & & 7 \\
\hline 558 & NSF & Mar-91 & Aug-94 & Lawrence & KS & UOF KANSAS CTR FOR $\bar{R}$ & Edlnst & 183.503 & 73.547 & & & & 7 \\
\hline 559 & NSF & Jun-91 & Nov-93 & State College & PA & PA ST U UNIVERSITY PAR & Edinst & \begin{tabular}{|l|}
149.788 \\
\end{tabular} & 69.261 & & & & 7 \\
\hline 560 & NSF & Aug-91 & Jan-95 & Tucson & $A \bar{Z}$ & UNIVERSITY OF ARZZONA & Edhst & 139.324 & 66.785 & 60.445 & 60.445 & 0 & 7 \\
\hline 561 & NSF & Apr-91 & Mar-95 & West Lafayette & IN & PURDUE UNIV RESEARCF & Edlnst & 136 & 68 & & & & 7 \\
\hline 562 & NSF & May-91 & Odt-94 & Atlanta & $\sqrt{G A}$ & GEORGIA TECH RESEARC & Edinst & 249.827 & 61.914 & & & & 7 \\
\hline 563 & NSF & Apr-91 & Mar-94 & San Diego & CA & UOF CALS & Edlnst & 510.979 & 265.745 & & & & 7 \\
\hline 564 & NSF & Juk-90 & Apr-96 & Boca Raton & $\mathrm{FL}$ & FLORIDA A TLANTIC UNIV & Edinst & 148.942 & 62.5 & 62.5 & 62.5 & & $\overline{7}$ \\
\hline 565 & NSF & Sep-90 & Aug-95 & Berkeley & CA & UOF CAL B & Edlnst & 251.75 & 55.25 & 51.5 & 51.5 & & 7 \\
\hline 566 & NSF & Sep-90 & Feb-96 & Satt Lake City & UT & UNIVERSITY OF UTAH & Edlnst & 254.894 & 45.833 & 25 & 25 & & 7 \\
\hline 567 & NSF & Oct-90 & Sep-95 & thaca & NY & CORNELL UNIVERSITY-ER & PriEdlnst & 146.158 & 70.025 & 88.916 & 88.916 & & 7 \\
\hline 568 & NSF & Jul-90 & Jun-94 & Madison & WI & UOF WISCONSIN MADISC & Edinst & 155 & 46.667 & 72.5 & 72.5 & 0 & 8 \\
\hline 569 & NSF & Jul-90 & Doc-95 & Buffalo & NY & SUNY BUFFALO & Edlnst & 139.53 & 59.01 & 62.5 & 62.5 & 0 & 6 \\
\hline 570 & NSF & Jul-90 & Jun-95 & Madison & WI & UOF WISCONSIN MADISC & Edlnst & 150 & 81.25 & 100 & 100 & 0 & 7 \\
\hline 571 & NSF & Aug-90 & Jan-96 & Palo Allo & CA & STANFORD UNIVERSITY & PriEdinst & 187.5 & 62.5 & 62.5 & 62.5 & 0 & 7 \\
\hline 572 & NSF & Aug-90 & Jul-97 & State College & PA & PA ST U UNIVERSITY PAR & Edlnst & 170 & 60.833 & 110 & 110 & 0 & 6 \\
\hline 573 & NSF & Sep-90 & Jan-94 & Notre Dame & IN & UNIVERSITY OF NOTRE D & PriEdlnst & 172.9 & 10 & & & & 7 \\
\hline 574 & NSF & Dec-91 & Nov-94 & Rochester & MI & OAKLAND UNIVERSITY & Edlnst & \begin{tabular}{|l|}
118.794 \\
\end{tabular} & 59.397 & & & & 7 \\
\hline 575 & NSF & Jul-92 & Doc-94 & East Hartiord $(\pi$ & ICT & NO PERFORMER INFO AV & NotSpec & 248.879 & 248.879 & & & & 7 \\
\hline 576 & NSF & Apr-91 & Mar-94 & San Diego & $C A$ & U OF CAL SAN DIEGO & Edlinst & 504.452 & 248.96 & & & & 7 \\
\hline 577 & NSF & Jul-91 & \begin{tabular}{|l|} 
Jun-94 \\
\end{tabular} & & OK & UOF OKLAHOMA & Edhst & 182.523 & 30.841 & 2.523 & 2.523 & 0 & 6 \\
\hline 578 & NSF & $\operatorname{Jan-92}$ & Jun-94 & Pittsburgh & PA & UOF PITTSBURGH & PriEdinst & 106.99 & 53.495 & & & & 7 \\
\hline 579 & NSF & Dec-91 & Nov-94 & Boca Raton & $\mathrm{FL}$ & FLORIDA ATLANTIC UNIV & Edlnst & 100.718 & 50.359 & & & & 7 \\
\hline 580 & NSF & Mar-91 & Aug-94 & Tuscaboosa & $A L$ & UOF ALABAMA TUUSCALO & Edinsi & 90 & 32.5 & & & & 7 \\
\hline 581 & NSF & Aug-91 & Jul-94 & Urbana & IIL & U OF ILLINOIS URBANA & Edlnst & 263.476 & 132.804 & & & & 7 \\
\hline 582 & NSF & Jun-92 & May-95 & Evanston & IL & NORTHWESTERN UNIVEF & PriEdinst & 16.825 & 16.825 & & & & 7 \\
\hline 583 & NSF & Jan-92 & Doc-94 & Houghton & MI & MICH TECHNOLOGICAL U & Edlnst & 78.21 & 39.105 & & & & 7 \\
\hline 584 & NSF & Mar-92 & Aug-95 & Laramie & WY & UNIVERSITY OF WYOMIN & Edlnst & 3000 & 1000 & 1000 & 1000 & & 7 \\
\hline 585 & NSF & Dec-91 & Nov-93 & Satt Lake City & UT & UNIVERSITY OF UTAH & Edlnst & 18 & 18 & & & & 7 \\
\hline
\end{tabular}




\begin{tabular}{|c|c|c|c|c|c|c|}
\hline & Agency & Bureau & Program & Projoct & Award Number & $\begin{array}{l}\text { Award } \\
\text { Type }\end{array}$ \\
\hline 586 & NSF & ENG & Civil \& mechanical systems (CMS) & Geomechanical/Geotech \& Geo-Environmental & 9109183 & $E / G$ \\
\hline 587 & NSF & ENG & Civil \& mechanical systems (CMS) & Architectural \& Mechanical Systems & 9109322 & $E / G$ \\
\hline 588 & NSF & ENG & Civil \& mochanical systems (CMS) & Siting \& Geotechnical Systems & 9110381 & $E / G$ \\
\hline 589 & NSF & ENG & Design, manufacture \& industrial innovation & SBIR-Phase II & 9110387 & $E / G$ \\
\hline 590 & NSF & ENG & Civil \& mochanical systems (CMS) & Nafural \& Man-Made Hazard Mitigation & 9110472 & $E / G$ \\
\hline 591 & NSF & ENG & Civil \& mechanical systems (CMS) & Structural Systems \& Construction Processes & 9110900 & $E / G$ \\
\hline 592 & NSF & ENG & Bioengineering \& environmental systems (B & Environmental Engineering Systems & 9112148 & $E / G$ \\
\hline 593 & NSF & ENG & Civil \& mochanical systems (CMS) & Architectural \& Mechanical Systems & 9112962 & $E / G$ \\
\hline 594 & NSF & ENG & Civil \& mechanical systems (CMS) & Structural Systems \& Construction Processes & 9113127 & $E / G$ \\
\hline 595 & NSF & ENG & Civil \& mochanical systems (CMS) & Architectural \& Mechanical Systems & 9113394 & $E / G$ \\
\hline 596 & NSF & ENG & Civil \& mechanical systems (CMS) & Large Structural \& Building Systems & 9113680 & $E / G$ \\
\hline 597 & NSF & ENG & Civil \& mochanical systems (CMS) & Natural \& Man-Made Hazard Mitigation & 9114006 & $E / G$ \\
\hline 598 & NSF & ENG & Civil \& mechanical systems (CMS) & Structural Systems \& Construction Processes & 9114095 & $E / G$ \\
\hline 599 & NSF & ENG & Civil \& mochanical systems (CMS) & Large Structural \& Building Systems & 9114188 & $E / G$ \\
\hline 600 & NSF & ENG & Civil \& mechanical systems (CMS) & Large Structural \& Building Systems & 9114238 & $E / G$ \\
\hline 601 & NSF & ENG & Civil \& mechanical systems (CMS) & Large Structural \& Building Systems & 9114313 & $E / G$ \\
\hline 602 & NSF & ENG & Civil \& mechanical systems (CMS) & Solid Mechanics & 9114360 & $E / G$ \\
\hline 603 & NSF & ENG & Civil \& mochanical systems (CMS) & Large Structural \& Building Systems & 9114459 & $E / G$ \\
\hline 604 & NSF & ENG & Civil \& mechanical systems (CMS) & Large Structural \& Building Systems & 9114476 & $E / G$ \\
\hline 605 & NSF & ENG & Civil \& mechanical systems (CMS) & Large Structural \& Building Systems & 9114511 & EG \\
\hline 606 & NSF & ENG & Civil \& mechanical systems (CMS) & Siructural Systems \& Construction Processes & 9114543 & $E / G$ \\
\hline 607 & NSF & ENG & Civil \& mechanical systems (CMS) & Large Siructural \& Building Systems & 9114679 & $E / G$ \\
\hline 608 & NSF & ENG & Civil \& mechanical systems (CMS) & Siting \& Geotechnical Systems & 9114895 & $E / G$ \\
\hline 609 & NSF & SBE & International cooperative scientific activities & Intemational activities - Other & 9114971 & $E / G$ \\
\hline 610 & NSF & ENG & Civil \& mochanical systems (CMS) & Geomochanical/Geolech \& Geo-Environmental & 9115197 & $E / G$ \\
\hline 611 & NSF & ENG & Civil \& mechanical systems (CMS) & Geomechanical/Geotech \& Geo-Environmental & 9115315 & $E / G$ \\
\hline 612 & NSF & ENG & Civil \& mechanical systems (CMS) & Siting \& Geotechnical Systems & 9115422 & $E / G$ \\
\hline 613 & NSF & SBE & International cooperative scientific activities & Intemational activities - Other & 9115553 & $E / G$ \\
\hline 614 & NSF & SBE & International cooperative scientific activities & Intemational activities - Other & 9115880 & $E / G$ \\
\hline 615 & NSF & CSE & Information, robotics \& intelligent systems (1) & Knowledge Models \& Cognitive Systems & 9116399 & $E / G$ \\
\hline 616 & NSF & ENG & Civil \& mechanical systems (CMS) & Large Structural \& Building Systems & 9116411 & $E / G$ \\
\hline 617 & NSF & CSE & Information, robotics \& intelligent systems (1) & Database \& Expert Systems & 9116646 & $E / G$ \\
\hline 618 & NSF & ENG & Civil \& mechanical systems (CMS) & Structural Systems \& Construction Processes & 9116666 & $E / G$ \\
\hline 619 & NSF & ENG & Civil \& mechanical systems (CMS) & Siting \& Geotechnical Systems & 9116714 & $E / G$ \\
\hline 620 & NSF & ENG & Civil \& mechanical systems (CMS) & Architectural \& Mechanical Systems & 9117049 & $E / G$ \\
\hline 621 & NSF & ENG & Bioengineering \& environmental systems (B & Environmental Engineering Systems & 9117267 & $E / G$ \\
\hline 622 & NSF & ENG & Civil \& mechanical systems (CMS) & Earthquake Systems Integration & 9117732 & $E / G$ \\
\hline 623 & NSF & ENG & Civil \& mechanical systems (CMS) & Large Structural \& Building Systems & 9118769 & $E / G$ \\
\hline 624 & NSF & ENG & Civil \& mechanical systems (CMS) & Architectural \& Mechanical Systems & 9120058 & $E / G$ \\
\hline 625 & NSF & ENG & Civil \& mochanical systems (CMS) & Architectural \& Mechanical Systems & 9120096 & $E / G$ \\
\hline 626 & NSF & ENG & Civil \& mechanical systems (CMS) & Architectural \& Mechanical Systems & 9120128 & $E / G$ \\
\hline 627 & NSF & ENG & Civil \& mechanical systems (CMS) & Architectural \& Mechanical Systems & 9120201 & $E / G$ \\
\hline 628 & NSF & ENG & Civil \& mechanical systems (CMS) & Architectural \& Mechanical Systems & 9120214 & $E / G$ \\
\hline 629 & NSF & ENG & Civil \& mechanical systems (CMS) & Architectural \& Mechanical Systems & 9120281 & $\bar{E} / \bar{G}$ \\
\hline 630 & NSF & ENG & Civil \& mechanical systems (CMS) & Architectural \& Mechanical Systems & 9120388 & $E / G$ \\
\hline 631 & NSF & ENG & Civil \& mechanical systems (CMS) & Earthquake Syslems integration & 9120397 & $E / G$ \\
\hline 632 & NSF & ENG & Civil \& mochanical systems (CMS) & Siting \& Geolechnical Systems & 9121292 & $E / G$ \\
\hline 633 & NSF & ENG & Civil \& mechanical systems (CMS) & Structural Systems \& Construction Processes & 9121342 & $E / G$ \\
\hline 634 & NSF & ENG & Civil \& mechanical systems (CMS) & Architectural \& Mechanical Systems & 9121443 & $E / G$ \\
\hline 635 & NSF & ENG & Civil \& mochanical systems (CMS) & Structural Systems & 9121943 & $E / G$ \\
\hline 638 & NSF & ENG & Civil \& mechanical systems (CMS) & Architectural \& Mechanical Systems & 9122046 & $E / G$ \\
\hline 637 & NSF & ENG & Civil \& mechanical systems (CMS) & Structural Systems & 9122704 & $E / G$ \\
\hline 638 & NSF & ENG & Civil \& mechanical systems (CMS) & Large Structural \& Building Systems & 9122747 & $E / G$ \\
\hline 638 & NSF & ENG & Civil \& mechanical systems (CMS) & Architectural \& Mechanical Systems & 9122922 & $E / G$ \\
\hline 640 & NSF & ENG & Civil \& mechanical systems (CMS) & Architectural \& Mochanical Systems & 9122950 & $E / G$ \\
\hline 641 & NSF & ENG & Civil \& mechanical systems (CMS) & Architoctural \& Mechanical Systems & 9123015 & $E / G$ \\
\hline 642 & NSF & ENG & Civil \& mechanical systems (CMS) & Aschitectural \& Mechanical Systems & 9123954 & $E / G$ \\
\hline 643 & NSF & ENG & Civa \& mochanical systems (CMS) & Large Structural \& Building Systems & 9157050 & $E / G$ \\
\hline 644 & NSF & ENG & Civil \& mechanical systems (CMS) & Structural Systems \& Construction Processes & 9157080 & $E / G$ \\
\hline 645 & NSF & ENG & Electrical \& communications systems (ECS & Systems Theory & 9157835 & $\bar{E} \bar{G}$ \\
\hline 646 & NSF & ENG & Civil \& mechanical systems (CMS) & Architectural \& Mechanical Systems & 9157942 & $E / G$ \\
\hline 647 & NSF & ENG & Design, manufacture \& industrial innovation & Production Systems & 9158136 & EJG \\
\hline 648 & NSF & ENG & Civil \& mechanical systems (CMS) & Large Structural \& Building Systems & 9158152 & E/G \\
\hline 649 & NSF & ENG & Engineering education \& centers (EEC) & Engineering education \& centers - Other & 9200117 & $E / G$ \\
\hline 650 & NSF & ENG & Engineering education \& centers (EEC) & Engineening education \& centers - Other & 9200139 & $E / G$ \\
\hline
\end{tabular}




\begin{tabular}{|c|c|c|c|c|c|c|c|c|c|c|c|c|c|}
\hline & Agency & $\begin{array}{l}\text { Start } \\
\text { Date }\end{array}$ & $\begin{array}{l}\text { End } \\
\text { Date }\end{array}$ & $\begin{array}{c}\text { Place of } \\
\text { Performance }\end{array}$ & $\begin{array}{l}\frac{9}{8} \\
0 \\
0 \\
0\end{array}$ & Performer Name & \begin{tabular}{c|c} 
Perf \\
Type
\end{tabular} & $\begin{array}{c}\text { Total } \\
\text { Award } \\
\text { Amount }\end{array}$ & $\begin{array}{c}\text { Average } \\
\text { Annual } \\
\text { Funding }\end{array}$ & $\begin{array}{l}\text { FY } \\
\text { Total } \\
\text { Amt }\end{array}$ & $\begin{array}{c}\text { FY Fod } \\
\text { Amt }\end{array}$ & \begin{tabular}{|c|} 
FY \\
Non- \\
Fed \\
Amt \\
\end{tabular} & $\begin{array}{c}\text { Prod. } \\
*\end{array}$ \\
\hline 586 & NSF & Sep-91 & Aug-94 & Amherst (censu & $\overline{M A}$ & UOF MASSACHUSETTS A & Edinst & 69.981 & 10 & & & & 7 \\
\hline 587 & NSF & May-91 & Oci-95 & Boulder & $\mathrm{CO}$ & ATKINSON-NOLAND ASSO & IndBus & 335 & 78.619 & & & & 7 \\
\hline 588 & NSF & Feb-92 & Jul-94 & Piscalaway (To & $\mathrm{NW}$ & NO PERFORMER INFO AV & NotSpec & 114.54 & 114.54 & & & & 7 \\
\hline 589 & NSF & Aug-93 & Jan-95 & Lithonia & $\widehat{G A}$ & EARTHWATER CORPORA & IndBus & 250 & 129 & 118.02 & 118.02 & 0 & 7 \\
\hline 590 & NSF & Jul-91 & Dec-93 & Coral Gables & $F L$ & UNIVERSITY OF MIAMI & PriEdinst & 66.293 & 9 & & & & 7 \\
\hline 591 & NSF & Jul-91 & Jun-94 & Slorrs & CT & UNIV OF CONNECTICUT & Edinst & 69.92 & 10 & & & & 7 \\
\hline 592 & NSF & Sep-91 & May-94 & Beaverton & OR & NO PERFORMER INFO AV & NotSpec & 158.462 & 75.425 & & & & 7 \\
\hline 593 & NSF & Jul-91 & Doc-94 & Potsdam & NY & CLARKSON UNIVERSITY & PriEdlnst & 163.199 & 12.5 & & & & 7 \\
\hline 594 & NSF & Oct-91 & Jun-94 & New York & NY & COLUMBIA UNIVERSITY & PriEdlnst & 120 & 59.255 & & & & 7 \\
\hline 595 & NSF & Nov-91 & Mar-94 & Berkeley & CA & U OF CAL BERKELEY & Edlnst & 40 & 40 & & & & 7 \\
\hline 596 & NSF & May-91 & Oct-93 & Boulder & $\mathrm{CO}$ & U OF COLORADO BOULDE & Edinst & 78.889 & 9.999 & & & & 7 \\
\hline 597 & NSF & Nov-91 & Apr-95 & Bantimore & MD & JOHNS HOPKINS UNIVER & PriEdinst & 295.127 & 98.376 & 63.541 & 63.541 & 0 & 7 \\
\hline 598 & NSF & Nov-91 & Oct-94 & Stanford & $\mathrm{CA}$ & STANFORD UNIVERSITY & PriEdinst & 140.02 & 140.02 & & & & 8 \\
\hline 599 & NSF & Dec-91 & May-95 & Washington & $\overline{D C}$ & CATHOLIC UNIVERSITY & PriEdinst & 104.235 & 52.118 & & . & & 7 \\
\hline 600 & NSF & Jan-92 & Jun-95 & Philadelphia & $\overline{P A}$ & DREXEL UNIVERSITY & PriEdInst & 259.969 & 86.656 & 78.717 & 78.717 & 0 & 7 \\
\hline 601 & NSF & Jan-92 & Jun-94 & Urbana & II & U OF ILLINOIS URBANA & EdInst & 32.366 & 32.366 & & & & 7 \\
\hline 602 & NSF & Apr-92 & Sep-95 & Urbana & IL & U OF ILI URBANA-CHAMP & Edlnst & 180 & 60 & 48.133 & 48.133 & 0 & 7 \\
\hline 603 & NSF & $\operatorname{Jan}-92$ & Jun-95 & Oakland & $P A$ & CARNEGIE MELLON UNIVE & PriEdInst & 215.937 & 71.979 & 74.194 & 74.194 & 0 & 5 \\
\hline 604 & NSF & Apr-92 & Mar-95 & Evanston & II & NORTHWESTERN UNIVER & PriEdlnst & 284.902 & 94.967 & 97.102 & 97.102 & 0 & 7 \\
\hline 605 & NSF & May-91 & Apr-95 & Boulder & $\mathrm{CO}$ & ATKINSON-NOLAND ASSO & IndBus & 213.281 & 48.101 & 71.299 & 71.299 & 0 & 7 \\
\hline 606 & NSF & Oct-91 & Sep-94 & Houston & $\overline{T X}$ & U OF HOUSTON & Edlnst & 132.7 & 20.694 & & & & 7 \\
\hline 607 & NSF & Jan-92 & Sep-94 & Columbia & MO & U OF MISSOURI COLUMBI & Edlnst & 50.968 & 50.968 & & & & $\overline{7}$ \\
\hline 608 & NSF & Feb-92 & Jan-95 & Philadelphia & PA & DREXEL UNIVERSITY & PriEdInst & 127.469 & 127.469 & & & & 7 \\
\hline 609 & NSF & Mar-92 & Feb-95 & Baltimore & MD & JOHNS HOPKINS UNIVER & PriEdlnst & 164.7 & 164.7 & & & & 7 \\
\hline 610 & NSF & OA-91 & Mar-94 & Philadelphia & PA & DREXEL UNIVERSITY & PriEdInSt & 82.5 & 90.958 & & & & 7 \\
\hline 611 & NSF & Nov-91 & Apr-95 & Madison & WI & U OF WISCONSIN MADISC & Edinst & 117.58 & 58.79 & & & & 7 \\
\hline 612 & NSF & $\operatorname{Jan}-92$ & Jul-95 & Pasadena & CA & CALIFORNIA INST OF TEC & PriEdlnst & 397.103 & 156.01 & 209.14 & 209.14 & 0 & 7 \\
\hline 613 & NSF & May-92 & Apr-95 & San Diego & CA & U OF CAL SAN DIEGO & Edlnst & 14.25 & $1 \overline{4.25}$ & & & & $\overline{7}$ \\
\hline 614 & NSF & Jun-92 & Nov-95 & Pittsburgh & $\overline{P A}$ & U OF PITTSBURGH & PriEdlnst & 12.65 & 12.65 & & & & 7 \\
\hline 615 & NSF & Mar-92 & Aug-95 & Palo Alto & $\mathrm{CA}$ & STANFORD UNIVERSITY & \begin{tabular}{|l|l|} 
PriEdinst \\
\end{tabular} & 321.435 & 115.478 & 140.85 & 140.85 & 0 & 8 \\
\hline 616 & NSF & Dec-91 & May-94 & Washington & $D C$ & NAT ACAD OF SCIENCES & NonprofNonE & 240 & 80 & 40 & 40 & 0 & 7 \\
\hline 617 & NSF & Sep-91! & Feb-95 & Palo Alio & $\mathrm{CA}$ & STANFORD UNIVERSITY & PriEdlnst & 650 & 300 & & & & 6 \\
\hline 618 & NSF & Dec-91 & May-94 & Amherst (censu & MA & U OF MASSACHUSETTS A & Edlnst & 119.7 & 119.7 & & & & 5 \\
\hline 619 & NSF & Mar-92 & Feb-95 & Los Angeles & CA & U OF SOUTHERN CALIFOR & PriEdlnst & 71.102 & 71.102 & & & & 6 \\
\hline 620 & NSF & Feb-92 & Jan-96 & Los Angeles & $\mathrm{CA}$ & U OF SOUTHERN CALIFOF & PriEdinst & 156 & 52 & 55.22 & 55.22 & 0 & 7 \\
\hline 621 & NSF & Mar-92 & Aug-94 & Gainesville & $F$ & U OF FLORIDA & Edlnst & 258.703 & 129.352 & & & & 7 \\
\hline 622 & NSF & Feb-92 & Jul-95 & Silver Spring & MD & BTI & indBus & 261.433 & 87.144 & 41.892 & 41.892 & 0 & 7 \\
\hline 623 & NSF & Nov-92 & Apr-96 & Long Beach & $\mathrm{CA}$ & CAL STATE ULONG BEAC & Edlnst & 266.645 & 87.104 & 87.964 & 87.964 & 0 & 7 \\
\hline 624 & NSF & Nov-91 & Feb-94 & Philadelphia & PA & DREXEL UNIVERSITY & PriEdlnst & 42.8 & 42.8 & & & & 7 \\
\hline 625 & NSF & Sep-91 & Aug-94 & Ann Arbor & MI & NO PERFORMER INFO AV & NotSpec & 140.049 & 67.907 & & & & 7 \\
\hline 626 & NSF & Feb-92 & $\operatorname{Jan}-95$ & Invine & CA & U OF CAL IRVINE & Edinst & 345.203 & 115.068 & 118.99 & 118.99 & 0 & 7 \\
\hline 627 & NSF & Aug-91 & Jul-94 & Austin & $T X$ & UOF TEXAS AUSTIN & Edlnst & 75.15 & 37.7 & & & & 7 \\
\hline 628 & NSF & Sep-91 & Feb-94 & San Francisco & CA & RUTHERFORD AND CHEK & IndBus & 34.816 & 13.653 & & & & 7 \\
\hline 629 & NSF & Sep-91 & Feb-95 & Ǘbana & IL & UOF ILL URBANA-CHAMP & Ẽdinst & 379.934 & 140.062 & & & & $\overline{7}$ \\
\hline 630 & NSF & Sep-91 & Aug-94 & Bethlehem & PA & LEHIGH UNIVERSITY & PriEdinst & 80.761 & 46.251 & & & & 7 \\
\hline 631 & NSF & Jan-92 & Dec-94 & Pasadena & $\mathrm{CA}$ & CALIFORNIA INST OF TEC & PriEdlnst & 66.901 & 66.901 & & & & 7 \\
\hline 632 & NSF & Jun-92 & May-95 & East Lansing & $\mathrm{MI}$ & MICHIGAN STATE UNIVER & \begin{tabular}{|l|} 
Edinst \\
\end{tabular} & 106.945 & 53.473 & & & & 7 \\
\hline 633 & NSF & Mar-92 & Oct-94 & Washington & DC & NATL INST OF BUILDING S & NonproNNonE & 10 & 10 & & & & 2 \\
\hline 634 & NSF & Apr-92 & Sep-94 & Tempe & $A Z$ & ARIZONA STATE UNIVERS & Edlinst & 86 & 43 & & & & 7 \\
\hline 635 & NSF & Apr-92 & Mar-95 & Berkeley & $\overline{C A}$ & U OF CAL BERKELEY & Edinst & 505.687 & 168.562 & 182 & 182 & 0. & 7 \\
\hline 636 & NSF & Jan-92 & Doc-94 & Invine & $\mathrm{CA}$ & UOF CAL IRVINE & Edins: & 250 & 125 & & & & 7 \\
\hline 637 & NSF & Jut-92 & Jun-95 & Ann Arbor & $\mathrm{MI}$ & NO PERFORMER INFO AV & NotSpec & 147.172 & 147.172 & & & & 7 \\
\hline 638 & NSF & Sep-91 & Feb-94 & Irvine & GA & UOF CAL IRVINE & Edlnst & 62.5 & 19.5 & & & & 7 \\
\hline 639 & NSF & Mar-92 & Aug-95 & Minneapolis & $\overline{M N}$ & U OF MINNESOTA-TWIN C & Edinst & 155.21 & 51.737 & 15.21 & 15.21 & 0 & 7 \\
\hline 640 & NSF & Mar-92 & Feb-95 & Austin & $T \bar{X}$ & U OF TEXAS AUSTIN & Edlnst & 240 & 80 & 80 & 80 & 0 & 7 \\
\hline 641 & NSF & Mar-92 & Aug-94 & Lincoln & NE & U OF NEBRASKA LINCOLN & Edinst & 260.034 & 130.017 & & & & 7 \\
\hline 642 & NSF & May-92 & Apr-94 & Recondo Beach & CA & CAISIS MANAGEMENT CO & frodBus & 126.598 & 126.598 & & & & 7 \\
\hline 643 & NSF & Aug-91 & $\operatorname{Jan}-97$ & Worcester & MA & WORCESTER POLYTECH & PriEdinst & 275 & 62.5 & 62.5 & 62.5 & 0 & 7 \\
\hline 644 & NSF & Jul-91 & Dec-95 & Blacksburg & VA & VA POLYTECHNIC INST \& & Edinst & 323.036 & 66.012 & 72.5 & 72.5 & 0 & 7 \\
\hline 645 & NSF & Aug-91 & Jul-94 & Los Angeles & $\mathrm{CA}$ & Ü OF SOUTHERN CALIFÖ & PriEdInst & 87.5 & 62.5 & & & & 8 \\
\hline 646 & NSF & Jul-91 & Dec-95 & Chicago & IL & ILLINOIS INST OF TECH & PriEdInst & 212.5 & 62.5 & & & & 7 \\
\hline 647 & NSF & Sep-91 & Aug-96 & Madison & W! & U OF WISCONSIN MADISO & Edlnst & 280 & 64.167 & 62.5 & 62.5 & 0 & 5 \\
\hline 648 & NSF & Sep-91 & Feb-96 & Seatlle & WA & U OF WASHINGTON & Edinst & 212.5 & 62.5 & 62.5 & 62.5 & 0 & 7 \\
\hline 649 & NSF & May-92 & Oct-93 & Albuquerque & NM & UNIVERSITY OF NEW MEX & Edinst & 39.923 & 39.923 & & & & 3 \\
\hline 650 & NSF & May-92 & Oct-95 & Brooklyn & NY & POLYTECHNIC UNIVERSIT & PriEdinst & 161.597 & 53.866 & 57.053 & 57.053 & 0 & 3 \\
\hline
\end{tabular}




\begin{tabular}{|c|c|c|c|c|c|c|}
\hline & Agency & Bureau & Program & Project & Award Number & $\begin{array}{c}\text { Award } \\
\text { Type }\end{array}$ \\
\hline 651 & NSF & ENG & Civil \& mechanical systems (CMS) & Large Structural \& Building Systems & 9200605 & $E / G$ \\
\hline 652 & NSF & ENG & Civil \& mechanical systems (CMS) & Large Structural \& Building Systems & 9200829 & $E / G$ \\
\hline 653 & NSF & ENG & Civil \& mechanical systems (CMS) & Geomechanical/Gootech \& Goo-Environmental & 9200892 & $E / G$ \\
\hline 654 & NSF & ENG & Civil \& mechanical systems (CMS) & Architectural \& Mochanical Systems & 9201437 & $E / G$ \\
\hline 655 & NSF & ENG & Civil \& mechanical systems (CMS) & Sructural Systems & 9201546 & $E / G$ \\
\hline 656 & NSF & ENG & Civil \& mochanical systems (CMS) & Earthquake Systems Integration & 9201553 & $E / G$ \\
\hline 657 & NSF & ENG & Civil \& mechanical systems (CMS) & Siructural Systems \& Construction Procosses & 9201687 & $E / G$ \\
\hline 658 & NSF & ENG & Civil \& mechanical systems (CMS) & Large Structural \& Building Systems & 9201697 & $E / G$ \\
\hline 659 & NSF & ENG & Civil \& mechanical systems (CMS) & Natural \& Man-Made Hazard Mitigation & 9201787 & $E / G$ \\
\hline 660 & NSF & ENG & Civil \& mochanical systems (CMS) & Structural Systems & 9201812 & $E / G$ \\
\hline 661 & NSF & ENG & Civil \& mechanical systems (CMS) & Large Structural \& Building Systems & 9201908 & $E / G$ \\
\hline 662 & NSF & ENG & Civil \& mechanical systems (CMS) & Structural Systems \& Construction Processes & 9201944 & $E / G$ \\
\hline 663 & NSF & ENG & Civil \& mechanical systems (CMS) & Natural \& Man-Made Hazard Mitigation & 9201962 & $E / G$ \\
\hline 664 & NSF & ENG & Civil \& mochanical systems (CMS) & Architectural \& Mechanical Systems & 9202039 & $E / G$ \\
\hline 665 & NSF & MPS & Mathematical sciences (DMS) & Algebra \& Number Theory & 9202071 & $E / G$ \\
\hline 666 & NSF & ENG & Civil \& mechanical systems (CMS) & Natural \& Man-Made Hazard Mitigation & 9202092 & $E / G$ \\
\hline 667 & NSF & ENG & Civil \& mechanical systems (CMS) & Structural Systems \& Construction Processes & 9202134 & $E / G$ \\
\hline 668 & NSF & ENG & Civil \& mechanical systems (CMS) & Structural Systems \& Construction Processes & 9202327 & $E / G$ \\
\hline 669 & NSF & ENG & Civil \& mechanical systems (CMS) & Structural Systems \& Construction Processes & 9202731 & $E / G$ \\
\hline 670 & NSF & ENG & Civil \& mechanical systems (CMS) & Large Structural \& Building Systems & 9202905 & $E / G$ \\
\hline 671 & NSF & ENG & Civil \& mechanical systems (CMS) & Structural Systems \& Construction Processes & 9203140 & $E / G$ \\
\hline 672 & NSF & ENG & Civil \& mechanical systems (CMS) & Large Structural \& Building Systems & 9203276 & $E / G$ \\
\hline 673 & NSF & ENG & Civil \& mechanical systems (CMS) & Structural Systems & 9203927 & $E / G$ \\
\hline 674 & NSF & ENG & Civil \& mechanical systems (CMS) & Architectural \& Mechanical Systems & 9204035 & $E / G$ \\
\hline 675 & NSF & ENG & Civil \& mechanical systems (CMS) & Geomechanical/Geotech \& Geo-Environmental & 9204433 & $E / G$ \\
\hline 676 & NSF & GEO & Earth sciences (EAR) & Geophysics & 9204643 & $E / G$ \\
\hline 677 & NSF & ENG & Civil \& mechanical systems (CMS) & Architectural \& Mechanical Systems & 9207158 & $E / G$ \\
\hline 678 & NSF & ENG & Civil \& mechanical systems (CMS) & Natural \& Man-Made Hazard Mitigation & 9209675 & $E / G$ \\
\hline 679 & NSF & ENG & Civil \& mechanical systems (CMS) & Large Structural \& Building Systems & 9209971 & $E / G$ \\
\hline 680 & NSF & ENG & Design, manufacture $\&$ industrial innovation & Computer Integrated Engineering & 9210018 & $E / G$ \\
\hline 681 & NSF & ENG & Civil \& mechanical systems (CMS) & Dynamic Systems \& Control & 9210178 & $E / G$ \\
\hline 682 & NSF & ENG & Civil \& mechanical systems (CMS) & Structural Systems & 9210585 & $E / G$ \\
\hline 683 & NSF & ENG & Civil \& mechanical systems (CMS) & Structural Systems \& Construction Processes & 9210721 & $E / G$ \\
\hline 684 & NSF & ENG & Civit \& mechanical systems (CMS) & Structural Systems \& Construction Processes & 9210894 & $E / G$ \\
\hline 685 & NSF & ENG & Civil \& mechanical systems (CMS) & Structural Systems \& Construction Processes & 9211063 & $E / G$ \\
\hline 686 & NSF & ENG & Civil \& mechanical systems (CMS) & Structural Systems \& Construction Processes & 9211421 & $E / G$ \\
\hline 687 & NSF & ENG & Design. manufacture \& industrial innovation & Computer Integrated Engineering & 9211657 & $E / G$ \\
\hline 688 & NSF & ENG & Civil \& mechanical systems (CMS) & Large Structural \& Building Systems & 9211678 & $E / G$ \\
\hline 689 & NSF & ENG & Bioengineering \& environmental systems (B & Environmental Engineering Systems & 9212059 & $E / G$ \\
\hline 690 & NSF & ENG & Civil \& mechanical systems (CMS) & Structural Systems \& Construction Processes & 9212318 & $E / G$ \\
\hline 691 & NSF & ENG & Civil \& mechanical sysiems (CMS) & Structural Syslems & 9212350 & $E / G$ \\
\hline 692 & NSF & ENG & Bioengineering \& environmental systems (E) & Environmental Engineering Systems & 9212505 & $E / G$ \\
\hline 693 & NSF & ENG & Civil \& mechanical systems (CMS) & Architectural \& Mechanical Systems & 9212519 & $E / G$ \\
\hline 694 & NSF & ENG & Civil \& mechanical systems (CMS) & Architectural \& Mechanical Systems & 9212700 & $E / G$ \\
\hline 695 & NSF & ENG & Civil \& mechanical systems (CMS) & Structural Systems \& Construction Processes & 9212733 & $E / G$ \\
\hline 696 & NSF & ENG & Civil \& mochanical sysiems (CMS) & Goomechanical/Geotech \& Geo-Environmental & 9212737 & $E / G$ \\
\hline 697 & NSF & ENG & Civil \& mechanical systems (CMS) & Architectural \& Mechanical Systems & 9213172 & $E / G$ \\
\hline 698 & NSF & ENG & Civil \& mechanical systems (CMS) & Structural Systems \& Construction Processes & 9213563 & $E / G$ \\
\hline 699 & NSF & ENG & Design. manufacture \& industrial innovation & Production Systems & 9213645 & $E / G$ \\
\hline 700 & NSF & SBE & International cooperative scientific activities & Easiem Europe & 9213675 & $E / G$ \\
\hline 701 & NSF & ENG & Crvil \& mechanical systems (CMS) & Architectural \& Mechanical Systems & 9213707 & $E / G$ \\
\hline 702 & NSF & ENG & Cnvil \& mechanical systems (CMS) & Nalural \& Man-Made Hazard Mitigation & 9214248 & $E / G$ \\
\hline 703 & NSF & SBE & Inlernational cooperative scientific activities & Intemational activities - Other & 9214251 & $E / G$ \\
\hline 704 & NSF & ENG & CMl \& mechanical systems (CMS) & Natural \& Man-Made Hazard Mitigation & 9214503 & $E / G$ \\
\hline 705 & NSF & ENG & CMl \& mechanical systems (CMS) & Structural Systems \& Construction Processes & 9214664 & $E / G$ \\
\hline 706 & NSF & ENG & Crvl \& mechanical systems (CMS) & Large Structural \& Building Systems & 9214738 & $E / G$ \\
\hline 707 & NSF & ENG & Cow \& mechanical systems (CMS) & Architectural \& Mechanical Systems & 9214754 & $E / G$ \\
\hline 708 & NSF & ENG & CMl \& mechanical systems (CMS) & Architectural \& Mechanical Systems & 9214999 & $E / G$ \\
\hline 709 & NSF & CSE & Informatıon, robotics \& intelligent systems (| & Interactive Systems & 9215178 & $E / G$ \\
\hline 710 & NSF & ENG & Civil \& mechanical systems (CMS) & Solid Mechanics & 9215299 & $E / G$ \\
\hline 711 & NSF & ENG & Civil \& mechanical systems (CMS) & Structural Systems \& Construction Processes & 9215412 & $E / G$ \\
\hline 712 & NSF & ENG & Civil \& mechanical systems (CMS) & Large Structural \& Building Systems & 9215521 & $E / G$ \\
\hline 713 & NSF & ENG & Civil \& mechanical systems (CMS) & Architectural \& Mechanical Systems & 9215522 & $E / G$ \\
\hline 714 & $\overline{N S} \bar{F}$ & ENG & Civil \& mechanical systems (CMS) & Structural Systems \& Construction Processes & 9215567 & $E / G$ \\
\hline 715 & NSF & ENG & Civil \& mechanical systems (CMS) & Structural Systems \& Construction Processes & 9215722 & $E / G$ \\
\hline
\end{tabular}




\begin{tabular}{|c|c|c|c|c|c|c|c|c|c|c|c|c|c|}
\hline & Agency & $\begin{array}{l}\text { Start } \\
\text { Date }\end{array}$ & $\begin{array}{l}\text { End } \\
\text { Date }\end{array}$ & $\begin{array}{c}\text { Place of } \\
\text { Performance }\end{array}$ & \begin{tabular}{l}
$:$ \\
\multirow{3}{*}{} \\
0 \\
0 \\
0
\end{tabular} & Performer Name & $\mid \begin{array}{lll}\text { Porf } & & \frac{\Sigma}{\Phi} \\
\text { Type } & \bar{\Xi} & \frac{Q}{\sigma} \\
\end{array}$ & $\begin{array}{c}\text { Total } \\
\text { Award } \\
\text { Amount }\end{array}$ & $\begin{array}{c}\text { Average } \\
\text { Annual } \\
\text { Funding }\end{array}$ & $\begin{array}{l}\text { FY } \\
\text { Total } \\
\text { Amrt }\end{array}$ & $\begin{array}{c}\text { FY Fed } \\
\text { Amt }\end{array}$ & $\begin{array}{c}\text { FY } \\
\text { Non- } \\
\text { Fod } \\
\text { Amt }\end{array}$ & $\begin{array}{c}\text { Prod } \\
*\end{array}$ \\
\hline 651 & INSF & Jan-92 & Jun-95 & Boulder & $\mathrm{CO}$ & ATKINSON-NOLAND ASSO & IndBus & \begin{tabular}{|r|}
50 \\
\end{tabular} & \begin{tabular}{|r|}
50 \\
\end{tabular} & & & & 7 \\
\hline 652 & INSF & Nov-92 & Apr-96 & Norman & OK & U OF OKLAHOMA & Edlnst & 52.664 & 26.332 & 0 & 0 & 0 & 7 \\
\hline 653 & NSF & Jut-92 & Dec-94 & Golden & $\mathrm{CO}$ & COLORADO SCHOOL OF N & VEdinst & 78.279 & 39.14 & & & & 7 \\
\hline 654 & NSF & Jun-92 & $\mathrm{Nov}-94$ & IUtbana & IL & U OF ILL URBANA-CHAMP & Edinst & 179.995 & 59.998 & 62.508 & 62.508 & 이 & 7 \\
\hline 655 & NSF & Mar-92 & Feb-96 & Houston & TX & U OF HOUSTON UNIV PAR & Edinst & 157.906 & 157.906 & & & & 6 \\
\hline 656 & NSF & Feb-92 & Aug-94 & | Gaithersburg & MD & INIST & Oth & 40 & 20 & & & & 7 \\
\hline 657. & NSF & Jut-92 & Dec-94 & Raleigh & NC & NORTH CAROLINA STATE & Edinst & 104.001 & 36.709 & 26.651 & 26.651 & 0 & 8 \\
\hline 658 & NSF & Nov-92 & Apr-96 & Raleigh & NC & NORTH CAROLINA STATE & Edlnst & 137.608 & 46.395 & 47.498 & 47.498 & 0 & 8 \\
\hline 659 & NSF & Jul-92 & Dec-95 & Ann Arbor & $\mathrm{MI}$ & U OF MICHIGAN ANN ARBS & Edlnst & 329.98 & 109.993 & 116.81 & 116.81 & 0 & 7 \\
\hline 660 & NSF & Jul-92 & Jun-95 & Invine & $\mathrm{CA}$ & UOF CAL IRVINE & EdInst & 210 & 70 & 73.94 & 73.94 & 0 & 7 \\
\hline 661 & NSF & Dec-92 & May-96 & Reno & NV & U OF NEVADA RENO & Edlnst & 204.923 & 80.679 & 71.008 & 71.008 & 0 & 7 \\
\hline 662 & NSF & Aug-92 & Jan-95 & Blacksburg & VA & VA POLYTECHNIC INST \& & Edinst & 150 & 50 & 100 & 100 & 0 & 7 \\
\hline 663 & NSF & Jun-92 & Nov-95 & Boulder & $\mathrm{CO}$ & U OF COLORADO BOULDE & EdInst & 215.76 & 71.92 & 70.485 & 70.485 & 우 & 7 \\
\hline 664 & NSF & Jul-92 & Dec-94 & Atlanta & GA & GA TECH RES CORP - GIT & Edlnst & 180 & 90 & & & & 7 \\
\hline 665 & NSF & Aug-92 & Jan-96 & Boston & MA & NORTHEASTERN UNIVERS & PriEdlnst & 80 & 26.667 & 27 & 27 & 0 & 7 \\
\hline 666 & NSF & Sep-92 & Feb-95 & College Station & $T X$ & TEXAS A\&MU RESEARCH & EdInst & 290 & 100 & 108.76 & 108.76 & 0 & 7 \\
\hline 667 & NSF & Jul-92 & Dec-94 & Lafayette & IN & PURDUE UNIV RESEARCH & Edinst & 140.071 & 46.69 & 45.913 & 45.913 & 0 & 7 \\
\hline 668 & NSF & Aug-92 & Jul-95 & Buffalo & NY & SUNY BUFFALO & Edlnst & 85.892 & 28.631 & 28.977 & 28.977 & c & 7 \\
\hline 669 & NSF & Aug-92 & $\operatorname{Jan}-96$ & Norman & $\overline{\mathrm{OK}}$ & UOF OKLAHOMA & EdInst & 216.372 & 54.457 & 74.029 & 74.029 & 0 & $\overline{7}$ \\
\hline 670 & NSF & Nov-92 & Oct.94 & Gainesville & $\mathrm{FL}$ & U OF FLORIDA & Edlnst & 150 & 75 & 53.145 & 53.145 & 0 & 5 \\
\hline 671 & NSF & May-92 & Oct-94 & Seattle & WA & UOF WASHINGTON & Edlnst & 119.684 & 39.895 & 41.56 & 41.56 & 0 & 7 \\
\hline 672 & NSF & Dec-92 & Nov-95 & Coral Gables & $F L$ & UNIVERSITY OF MIAMI & PriEdlnst & 171.399 & 54.203 & & & & 7 \\
\hline 673 & NSF & Aug-92 & Jul-94 & Pasadena & $\mathrm{CA}$ & CALIFORNIA INST OF TEC & PriEdlnst & 59.756 & 59.756 & & & & 7 \\
\hline 674 & NSF & Apr-92 & Sep-94 & Blacksburg & VA & VIRGINIA POLYTECHNIC If & Edlnst & 2.75 & 2.75 & & & & 7 \\
\hline 675 & NSF & Nov-92 & Apr-96 & Urbana & IL & U OF ILL URBANA-CHAMP & Edlnst & 180.083 & 60.334 & 61.121 & 61.121 & 0 & 7 \\
\hline 676 & NSF & Jul-92 & Dec-93 & Chapel Hill & NC & U OF NC CHAPEL HILL & Edlnst & 45.396 & 45.396 & & & & 7 \\
\hline 677 & NSF & Sep-92 & Aug-94 & Seattle & WA & URBAN REGIONAL RESEA & IndBus & 79.806 & 79.806 & & & & 7 \\
\hline 678 & NSF & May-92 & OAd-93 & Lubbock & TX & TEXAS TECH UNIVERSITY & Edinsi & 17.902 & 17.902 & & & & 7 \\
\hline 679 & NSF & Aug-92 & Jut-94 & Lafayette & LA & U OF SOUTHWESTRN LOU & Edlnst & 16.984 & 16.984 & & & & 7 \\
\hline 680 & NSF & Sep-92 & Feb-95 & Tucson & $A Z$ & U OF ARLONA & Edlnst & 118.9 & 39.633 & 40 & 40 & 0 & 7 \\
\hline 681 & NSF & Sep-92 & Aug-96 & Cincinnati & $\mathrm{OH}$ & U OF CINCINNATI & Edlnst & 90 & 30 & 30 & 30 & 0 & 8 \\
\hline 682 & NSF & Jun-93 & May-96 & Houston & $\mathrm{TX}$ & WILLIAM MARSH RICE UN & PriEdlnst & 86.302 & 86.302 & & & & 7 \\
\hline 683 & NSF & Sep-92 & Feb-95 & College Park & MD & U OF MD COLLEGE PARK & Edlnst & 99.951 & 34.67 & 60 & 60 & 0 & 8 \\
\hline 684 & NSF & Mar-93 & Aug-96 & Notre Dame & IN & UNIVERSITY OF NOTRE D & PriEdlnst & 95.495 & 95.495 & & & & 7 \\
\hline 685 & NSF & Aug-92 & Jan-95 & Tempe & $A Z$ & ARIZONA STATE UNIVERS & Edlnst & 99.151 & 33.05 & 59.922 & 59.922 & 0 & 7 \\
\hline 686 & NSF & Sep-92 & Aug-95 & Austin & $\mathrm{TX}$ & U OF TEXAS AUSTIN & Edlnst & 100 & 46.667 & 60 & 60 & 0 & 8 \\
\hline 687 & NSF & Aug-92 & Jul-96 & Lubbock & $T X$ & TEXAS TECH UNIVERSITY & Edlnst & 89.732 & 29.911 & 24.014 & 24.014 & 0 & 8 \\
\hline 688 & NSF & Jan-93 & Dec-96 & thaca & NY & CORNELL UNIVERSITY-EN & PriEdinst & 282.99 & 73.051 & 69.969 & 69.969 & 0 & 7 \\
\hline 689 & NSF & Sep-92 & Aug-95 & Aloha & OR & OREGON GRAD INST OF S & PriEdinst & 94.19 & 31.397 & 29.931 & 29.931 & 0 & 7 \\
\hline 690 & NSF & Nov-92 & Apr-96 & Blacksburg & VA & VA POLYTECHNIC INST \& & Edinst & 224.994 & 79.985 & 78.51 & 78.51 & 0 & 7 \\
\hline 691 & NSF & Dec-92 & May-94 & Potsdam & NY & CLARKSON UNIVERSITY & PriEdlnst & 25.535 & 25.535 & & & & 7 \\
\hline 692 & NSF & Jul-92 & Dec-93 & Albuquerque & $\overline{N M}$ & UNIVERSITY OF NEW MEX & Edlnst & 10.6 & 10.6 & & & & 7 \\
\hline 693 & NSF & May-92 & Oct-93 & Richmond & $\mathrm{CA}$ & CUREE & NonprofiNonE & 36.855 & 36.855 & & & & 7 \\
\hline 694 & NSF & Jul-92 & Jun-94 & Notre Dame & IN & UNIVERSITY OF NOTRE D & PriEdinst & 36.837 & 18.419 & & & & 7 \\
\hline 695 & NSF & Dec-92 & May-96 & Albuquerque & NM & UNIVERSITY OF NEW MEX & Edlnst & 141.523 & 52.851 & 47.892 & 47.892 & 0 & 7 \\
\hline 696 & NSF & Nov-92 & Oct-95 & Berkeley & CA & U OF CAL BERKELEY & Edlnst & 180 & 60 & 60 & 60 & 0 & 7 \\
\hline 697 & NSF & Sep-92 & Feb-95 & Rolla & MO & U OF MISSOURI ROLLA & Edlnst & 248.508 & 82.836 & 55.579 & 55.579 & 0 & 7 \\
\hline 698 & NSF & Dec-92 & Nov-94 & State College & $\overline{P A}$ & PA ST U UNIVERSITY PAR & Edlnst & 104 & 104 & & & & 7 \\
\hline 699 & NSF & Jun-92 & May-94 & Tuscaloosa & $A L$ & NO PERFORMER INFO AV & NotSpec & 8.54 & 8.54 & & & & 5 \\
\hline 700 & NSF & Mar-93 & Aug-96 & State Colioge & PA & PAST U UNIVERSITY PAP & EdInst & 26.275 & 26.275 & & & & 7 \\
\hline 701 & NSF & May-93 & 0 ad-94 & Houston & $\mathrm{TX}$ & UOF HOUSTON & Edinst & 156.757 & 78.379 & 84.635 & 84.635 & 0 & 7 \\
\hline 702 & NSF & Jun-93 & Nov-96 & Seattle & WA & U OF WASHINGTON & Edlnst & 280.07 & 82.518 & 115.04 & 115.04 & 0 & 7 \\
\hline 703 & NSF & Sep-92 & Dec-93 & Lubbock & $T X$ & TEXAS TECH UNIVERSITY & Edhat & 19 & 19 & & & & 7 \\
\hline 704 & NSF & Dec-92 & May-97 & Burlington & $\sqrt{T}$ & U OF VERMONT & Edlnst & 271.397 & 77.347 & & & & 7 \\
\hline 705 & NSF & Sep-92 & Fob-96 & Rolla & MO & U OF MISSOURI ROLLA & Edlnst & 234.73 & 157.365 & & & & 7 \\
\hline 706 & NSF & Nov-92 & Oct-94 & Coral Gables & FL & UNIVERSITY OF MIAMI & PriEdlnst & 50.522 & 50.522 & & & & 7 \\
\hline 707 & NSF & Jan-93 & Dea-93 & LOS Angeles & $C A$ & SRE & IndBus & 25 & 25 & & & & 7 \\
\hline 706 & NSF & Feb-93 & Jul-94 & Emeryvile & $C A$ & CLADDING RES INST & IndBus & 65 & 65 & & & & 7 \\
\hline 709 & NSF & Apr-93 & Sep-94 & Washington & $D C$ & NAT ACAD OF SCIENCES & NonprofNonE & 34.71 & 34.71 & & & & 5 \\
\hline 710 & NSF & Sep-92 & Feb-94 & Evanston & IL & NOORTHWE'STERN UNIVEA & PriEdinst & 14.227 & 14.227 & & & & 7 \\
\hline 711 & NSF & Nov-92 & Apr-96 & Blacksburg & VA & VA POLYTECHNIC INST \& & Edlnst & 200 & 74.908 & 70.896 & 70.896 & 0 & 7 \\
\hline 712 & NSF & Mar-93 & Aug-96 & Brooklyn & NY & POLYTECHNIC UNIVERSIT & PriEdinst & 187.096 & 64.032 & 59.032 & 59.032 & 0 & 6 \\
\hline 713 & NSF & Aug-92 & Jan-94 & San Francisco & CA & EQE INTERNATIONAL INC & IndBus & 45.51 & 22.755 & & & & 7 \\
\hline 714 & NSF & Jan-93 & Jun-96 & Ames & IA & IOWA STATE UNIVERSITY & Edlnst & 207.261 & 77.779 & 75.188 & 75.188 & 0 & 7 \\
\hline 715 & NSF & Aug-92 & Jan-95 & Blacksburg & $\overline{\mathrm{VA}}$ & VA POLYTECHNIC INST \& & Edlnst & 160 & 53.333 & 100 & 100 & 0 & 7 \\
\hline
\end{tabular}




\begin{tabular}{|c|c|c|c|c|c|c|}
\hline & Agency & Bureau & Program & Project & Award Number & $\begin{array}{l}\text { Award } \\
\text { Type }\end{array}$ \\
\hline 716 & NSF & ENG & Engineering education \& conters (EEC) & Industry University Cooperative Research Cenle & 9215864 & \\
\hline 717 & NSF & ENG & Civil \& mechanical systems (CMS) & Siructural Systems \& Construction Processes & 9215935 & $E J G$ \\
\hline 718 & NSF & ENG & Civil \& mochanical systems (CMS) & Nalural \& Man-Made Hazard Mitigation & 9216233 & EJG \\
\hline 719 & NSF & ENG & Civil \& mechanical systems (CMS) & Siting \& Goolechnical Systems & 9216234 & $E / G$ \\
\hline 720 & NSF & ENG & Civil \& mechanical systems (CMS) & Earthquake Systems Integration & 9216881 & $E G$ \\
\hline 721 & NSF & SBE & International cooperative scientific activities & Americas & 9217433 & EJG \\
\hline 722 & NSF & $\mathrm{SBE}$ & International cooperative scientific activities & Americas & 9217441 & EJG \\
\hline 723 & NSF & SBE & International cooperative scientific activities & Japan & 9218165 & EIG \\
\hline 724 & NSF & ENG & Civil \& mochanical systems (CMS) & Structural Systems \& Consinuction Procosses & 9218182 & EJG \\
\hline 725 & NSF & ENG & Civil \& mochanical systems (CMS) & Siructural Systems & 9218229 & EJG \\
\hline 726 & NSF & ENG & Civil \& mechanical systems (CMS) & Natural \& Man-Made Hazard Mitigation & 9218343 & $E / G$ \\
\hline 727 & NSF & $S B E$ & International cooperative scientific activities & Japan & 9218743 & EjG \\
\hline 728 & NSF & ENG & Civil \& mechanical systems (CMS) & Natural \& Man-Made Hazard Mitigation & 9219921 & EIG \\
\hline 729 & NSF & SBE & International cooperative scientific activities & Intemational activities - Other & 9221217 & $E / G$ \\
\hline 730 & NSF & ENG & Civil \& mechanical systems (CMS) & Structural Systems & 9221314 & EJG \\
\hline 731 & NSF & ENG & Civil \& mechanical systems (CMS) & Architectural \& Mechanical Systems & 9221517 & $E J G$ \\
\hline 732 & NSF & ENG & Civil \& mech anical systems (CMS) & Architectural \& Mechanical Systems & 9221522 & $E / G$ \\
\hline 733 & NSF & ENG & Civil \& mechanical systems (CMS) & Architectural \& Mechanical Systems & 9221527 & $E G$ \\
\hline 734 & NSF & ENG & Civil \& mechanical systems (CMS) & Struclural Systems & 9221531 & EJG \\
\hline 735 & NSF & ENG & Civil \& mochanical systems (CMS) & Siructural Systems & 9221634 & EJG \\
\hline 736 & NSF & ENG & Civil \& mech anical systems (CMS) & Large Structural \& Building Systems & 9221977 & EJG \\
\hline 737 & NSF & ENG & Civil \& mechanical systems (CMS) & Large Structural \& Building Systems & 9222022 & EJG \\
\hline 738 & NSF & ENG & Bioengineering \& environmental systems (E & Ocoan Engineering Systems & 9222032 & $E / G$ \\
\hline 739 & NSF & ENG & Civil \& mechanical systems (CMS) & Large Structural \& Building Systems & 9222034 & $E / G$ \\
\hline 740 & NSF & ENG & Civil \& mechanical systems (CMS) & Structural Systems \& Consiruction Processos & 9222064 & $E G$ \\
\hline 741 & NSF & ENG & Civil \& mochanical systems (CMS) & Structural Systems & 9222110 & EJG \\
\hline 742 & NSF & ENG & Civil \& mechanical systems (CMS) & Structural Systems \& Construction Processes & 9222114 & EJG \\
\hline $743 t$ & NSF & ENG & Bioengineering \& environmental systems (E & Environmental Engineering Systems & 9222116 & $E / G$ \\
\hline 744 & NSF & ENG & Civil \& mochanical systems (CMS) & Structural Systems & 9222184 & EJG \\
\hline 745 & NSF & ENG & Bioengineering \& environmental systems (E & Environmental Engineering Systems & 9222559 & $E / G$ \\
\hline 746 & NSF & ENG & Civil \& mechanical systems (CMS) & Large Structural \& Building Systems & 9222581 & $E / G$ \\
\hline 747 & NSF & ENG & Civil \& mochanical systems (CMS) & Siting \& Geolechnical Systems & 9222669 & EJG \\
\hline 748 & NSF & ENG & Civil \& mochanical systems (CMS) & Architectural \& Mechanical Systems & 9222710 & $E / G$ \\
\hline 749 & NSF & SBE & International cooperafive scientific activities & East Asia \& Pacific & 9222933 & $E / G$ \\
\hline 750 & NSF & ENG & Civil \& mechanical systems (CMS) & Architectural \& Mechanical Systems & 9223234 & $E / G$ \\
\hline 7511 & NSF & ENG & Civil \& mechanical systems (CMS) & Earthquake Systems Integration & 9223453 & EJG \\
\hline 752 & NSF & ENG & Bioengineering \& environmental systems (B & Environmental Engineering Systems & 9223656 & $E / G$ \\
\hline 753 & NSF & ENG & Civil \& mechanical systems (CMS) & Earthquake Systems Integration & 9223680 & $E / G$ \\
\hline 754 & NSF & ENG & Civil \& mechanical systems (CMS) & Large Structural \& Building Systems & 9223778 & $E / G$ \\
\hline 755 & NSF & SBE & Social, behavioral \& economic research (SE & Ethies \& Values Studies & 9224037 & $E / G$ \\
\hline 756 & NSF & ENG & Civil \& mechanical systems (CMS) & Large Structural \& Building Systems & 9224316 & $E / G$ \\
\hline 757 & NSF & ENG & Civil \& mechanical systems (CMS) & Sitzing \& Gootechnical Systems & 9224479 & $E / G$ \\
\hline 758 & NSF & ENG & Civil \& mechanical systems (CMS) & Large Structural \& Building Systems & 9224531 & $E / G$ \\
\hline 759 & NSF & ENG & Electrical \& communications systems (ECS & Computational Engineering & 9224828 & $E / G$ \\
\hline 760 & NSF & ENG & Civil \& mechanical systems (CMS) & Natural \& Man-Made Hazard Mitigation & 9224837 & $E / G$ \\
\hline 761 & NSF & ENG & Civil \& mechanical systems (CMS) & Natural \& Man-Made Hazard Mitigation & 9224838 & $E / G$ \\
\hline 762 & NSF & ENG & \begin{tabular}{|l|} 
Civil \& mechanicel systems (CMS) \\
\end{tabular} & Siting \& Geotochnical Systems & 9224945 & $E / G$ \\
\hline 763 & NSF & ENG & Civil \& mechanical systems (CMS) & Earthquake Systems Integration & 9225056 & $E / G$ \\
\hline 764 & NSF & ENG & Civil \& mechanical systems (CMS) & Natural \& Man-Made Hazard Nitigation & 9225108 & $E / G$ \\
\hline 765 & NSF & EHR & Human resource development (HRD) & & 9252923 & $E / G$ \\
\hline 768 & NSF & ENG & Civil \& mechanical systems (CMS) & Solid Mochanies & 9253777 & $E / G$ \\
\hline 767 & NSF & ENG & Engineering education \& conters (EEC) & Engineering education \& conters - Other & 9256719 & $E / G$ \\
\hline 768 & NSF & ENE & Civil \& mechanical systems (CMS) & Siructural Sysiems \& Construction Processes & 9257231 & E/G \\
\hline 769 & NSF & ENG & Civit \& mechanical systems (CMS) & Large Structural \& Building Systems & 9257344 & $E / G$ \\
\hline 770 & NSF & ENG & Civil \& mechanical systems (CMS) & Siting \& Gootechnical Systems & 9257900 & $E / G$ \\
\hline$\pi 11$ & NSF & ENG & Civil \& mochanical systems (CMS) & Structural Systems \& Construction Processes & 9257933 & $E / G$ \\
\hline$\pi 2$ & NSF & ENG & Civil \& mechanical systems (CMS) & Large Structural \& Building Systems & 9258648 & $E / G$ \\
\hline$\pi 3$ & NSF & ENG & Design, manufecture \& industrial innovation & SBIR-Phase! & 9260109 & $E / G$ \\
\hline 774 & NSF & ENG & Design, manufacture \& incustrial mnovation & SBIR-Phase I & 9260918 & $E / G$ \\
\hline 775 & NSF & ENG & Civil \& mechanical systems (CMS) & Architectural \& Mechanical Systems & 9296139 & $E / G$ \\
\hline 776 & NSF & ENG & Civil \& mechanical systems (CMS) & Large Structural \& Building Systems & 9296190 & $E / G$ \\
\hline$\pi 77$ & NSF & ENG & \begin{tabular}{|l|} 
Civil \& mechanical systems (CMS) \\
\end{tabular} & Geomechanical/Geolech \& Geo-Environmental & $\$ 9300195$ & $E / G$ \\
\hline$\pi 78$ & NSF & ENG & Engineering education \& centers (EEC) & Engineering education \& centers - Other & 9300323 & $E / G$ \\
\hline 79 & NSF & ENG & $\begin{array}{l}\text { Civil \& mechanical systems (CMS) } \\
\end{array}$ & Structural Systems & 9300682 & $E / G$ \\
\hline 780 & $\mathrm{NSF}$ & ENG & Design, manufacture \& industrial innovation & Engineering Design & 9300742 & $E / G$ \\
\hline
\end{tabular}




\begin{tabular}{|c|c|c|c|c|c|c|c|c|c|c|c|c|c|}
\hline & Ageney & $\begin{array}{l}\text { Start } \\
\text { Date }\end{array}$ & $\begin{array}{l}\text { End } \\
\text { Date }\end{array}$ & $\begin{array}{c}\text { Place of } \\
\text { Performance }\end{array}$ & $\begin{array}{l}\frac{9}{5} \\
0 \\
0 \\
0\end{array}$ & Performer Name & 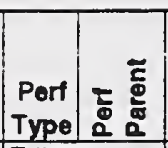 & \begin{tabular}{|c|} 
Total \\
Award \\
Amount \\
\end{tabular} & $\begin{array}{c}\text { Average } \\
\text { Annual } \\
\text { Funding }\end{array}$ & $\begin{array}{c}F Y \\
\text { Total } \\
\text { Amt }\end{array}$ & $\begin{array}{c}\text { FY Fod } \\
\text { Amt }\end{array}$ & $\begin{array}{c}\text { Fy } \\
\text { Non- } \\
\text { Fod } \\
\text { Amt }\end{array}$ & $\begin{array}{c}\text { Prod } \\
\vdots\end{array}$ \\
\hline 716 & NSF & Oat-92 & Mar-94 & Newark & NJ & NUIT & Edlnst & 150 & 137.5 & & & & \\
\hline 717 & NSF & Aug-92 & Jut-95 & Ann Arbor & MI & UOF MICHIGAN ANN ARBd & Edinst & 160 & 53.333 & 87.226 & 87.226 & 0 & 6 \\
\hline 718 & NSF & Jun-92 & May-94 & Uibana & IL & U OF ILLINOIS URBANA & Edlnst & 35.464 & 35.464 & & & & \\
\hline 719 & NSF & Jun-92 & Nov-93 & Troy & INY & NO PERFORMER INFO AV & NotSpec & 38.298 & 38.298 & & & & 7 \\
\hline 720 & NSF & Jun-93 & Nov-94 & Woodland Hins & $C A$ & MICHAEL E DURKIN ASSO & IndBus & 81.728 & 40.864 & 12 & 12 & 0 & 7 \\
\hline 721 & NSF & Apr-93 & Mar-95 & Boca Raton & $A$ & FLORIDA ATLANTIC UNIV & Edlnst & 19.55 & 19.55 & & & & \\
\hline 722 & NSF & Jan-93 & Dec-94 & Tampa & FL & U OF SOUTH FLORIDA & Edlnst & 4.832 & 4.832 & & & & \\
\hline 723 & NSF & Apr-93 & Mar-96 & State Colloge & PA & PAST U UNIVERSITY PAA & Edlnst & 13.595 & 13.595 & & & & 7 \\
\hline 724 & NSF & Doc-92 & May-94 & Rolla & MO & U OF MISSOURI ROLLA & Edlnst & 20 & 20 & & & & 7 \\
\hline 725 & NSF & Sep-92 & Feb-94 & Las Cruces & NM & NEW MEXICO ST UNIVERS & Edlnst & 49.962 & 24.981 & & & & \\
\hline 726 & NSF & Sep-92 & Aug-95 & Lubbock & $T X$ & TEXAS TECH UNIVERSITY & Edlnst & 134.138 & 51.897 & 45.208 & 45.208 & 0 & 7 \\
\hline 727 & NSF & Apr -93 & Mar-94 & Davis & $C A$ & UOF CAL DAVIS & Edinst & 11 & 11 & & & & \\
\hline 728 & NSF & Sep-92 & Feb-95 & Seattle & WA & UOF WASHINGTON & Edlnst & 107.997 & 35.999 & 35.999 & 35.999 & 0 & \\
\hline 729 & NSF & Mar-93 & Aug-94 & State Colloge & PA & PA ST U UNIVERSITY PAR & Edlnst & 58.936 & 58.936 & & & & \\
\hline 730 & NSF & Mar-93 & Aug-95 & Reno & NV & U OF NEV ADA RENO & Edlnst & 79.342 & 39.671 & 36.708 & 36.708 & 0 & 7 \\
\hline 731 & NSF & Jan-93 & Jun-95 & & WA & UOF WASHINGTON & Edlnst & 155.423 & 155.423 & & & & 7 \\
\hline 732 & NSF & Sep-92 & Aug-95 & Hous & $T X$ & WILLIAM MARSH RICE UN & PriEdinst & 100 & 200 & & & & 7 \\
\hline 733 & NSF & Sep-92 & Feb-94 & Los Ang & CA & NABBIH YOUSSEF \& ASSOC & indBus & 62 & 51.115 & & & & 7 \\
\hline 734 & NSF & Feb-93 & Jul-96 & Austin & $\overline{T X}$ & UUOF TEXAS AUSTIN & Edinst & 220 & 74.5 & 80 & 80 & 0 & 7 \\
\hline 735 & NSF & Nov-92 & Apr-95 & & $\mathrm{CA}$ & SAN JO & Edlnst & 107.31 & 107.31 & & & & \\
\hline 736 & NSF & Apr-93 & Sep-96 & Lawrence & KS & U OF KANSAS CT & Edlnst & 190.879 & 70.428 & & & & 6 \\
\hline 737 & NSF & Apr-93 & Sep-96 & Rolla & MO & U OF MISSOURI ROLLA & Edlnst & 149.051 & & 51.446 & 51.446 & 0 & \\
\hline 738 & NSF & Apr-94 & Mar-96 & College Station & $\mathrm{TX}$ & TEXAS ENGINEERING EX & Edinst & 239.098 & 75.472 & 75.472 & 75.472 & 0 & 7 \\
\hline 739 & NSF & Apr-93 & Sep-96 & & MO & UOF MIS & Edlnst & 278.423 & 98.195 & 72.115 & 72.115 & 0 & \\
\hline 740 & NSF & Mar-93 & Aug-96 & & VA & VAPO & Edlnst & 130 & 42.5 & 45 & 45 & 0 & \\
\hline 741 & NSF & Mar-93 & Feb-94 & & CA & UOFC & Edlnst & 56.59 & 56.59 & & & & 7 \\
\hline 742 & NSF & $b-93$ & Jan-96 & & OH & OHIO STATE UNIV RES FD & Edlnst & 132.533 & 41.932 & 44.833 & 44.833 & 0 & \\
\hline 743 & NSF & Mar-93 & Aug -96 & & $M A$ & MITT & PriEdinst & $280 . \overline{316}$ & & 94.77 & 94.77 & 0 & 7 \\
\hline 744 & NSF & Apr-93 & Sep-94 & Boston & MA & NORTI & PriEdlnst & 30.544 & 30.544 & & & & \\
\hline 745 & NSF & Sep-92 & Feb-94 & & CA & RRES IN & NonproiNonE & 12 & 12 & & & & 7 \\
\hline 746 & NSF & Jan-93 & Dec-93 & & SC & ITY & Edinst & 18.621 & 18.621 & & & & 7 \\
\hline 747 & NSF & $19-93$ & Jan-96 & & WA & WASH & Edlnst & 195.295 & 71.868 & 115.52 & 115.52 & 0 & \\
\hline 748 & NSF & pp-92 & & & NY & SUNY & Edinst & 29.992 & & & & & \\
\hline 749 & NSF & Apr-93 & Mar-94 & $\mathrm{BOC}$ & $F$ & FLOR & Edinst & 23.168 & 23.168 & & & & \\
\hline 750 & NSF & Jul-93 & In-96 & & CA & UOF & Edinst & 322.355 & 180 & & & & 7 \\
\hline 751 & NSF & Oct-93 & Mar-96 & & $C A$ & EART & NonprofNonE & 45 & 54.54 & 54.54 & 54.54 & 0 & 7 \\
\hline 752 & NSF & Sep-93 & Feb-96 & & $\mathbb{I N}$ & & EdInst & 225.235 & & 72.437 & 72.437 & 0 & \\
\hline 753 & NSF & May-93 & Apr-95 & & CA & TEC & PriEdInst & 148.113 & & & & & 6 \\
\hline 754 & NSF & Jul-93 & Jun-97 & & NY & $\mathrm{COR}$ & PriEdlnst & 282.99 & & 69.969 & 69.969 & 0 & \\
\hline 755 & NSF & Mar-93 & Feb-95 & & $O R$ & UOF & Edinst & 60.001 & & & & & 7 \\
\hline 756 & NSF & Jut-93 & Dec-95 & & NM & NEW & Edinst & 153.405 & & 60.814 & 60.814 & 0 & \\
\hline 757 & NSF & Aug-93 & Jut-96 & & CA & UOF & Edinst & 188.99 & & 72.863 & 72.863 & 0 & \\
\hline 758 & NSF & Apr-93 & Sep-95 & & NY & CORI & PriEdlns & & & 20 & 20 & 0 & \\
\hline $7 \overline{59}$ & NSF & $y-94$ & Oct-96 & & IL & $\bar{U} \overline{O F}$ & Ëdinst & & 37 & 54.437 & 54.437 & 0 & \\
\hline 760 & NSF & ov-92 & ct.94 & & $\mathrm{FL}$ & & Edinst & & & & & & 7 \\
\hline 761 & NSF & $c-92$ & Nov-93 & & $\mathrm{FL}$ & FLOF & Edlnst & & & & & & 7 \\
\hline 762 & NSF & $b-93$ & & & CA & UOF & PriEdlns & 782 & & & & & \\
\hline 763 & NSF & ar -93 & Feb-94 & & NY & $\mathrm{COR}$ & PriEdinst & 7 & 7 & & & & 7 \\
\hline 764 & NSF & $N-92$ & Apr-94 & ation & $T X$ & & EdInst & 50 & 50 & & & & \\
\hline 765 & NSF & Sep-92 & $6-95$ & & NC & NOR & Edlnst & 349.998 & & 75.86 & 75.86 & 0 & 7 \\
\hline 766 & NSF & $p-92$ & & & $\mathrm{PA}$ & & PriEdlns & 500 & & 100 & 100 & 0 & 5 \\
\hline 767 & NSF & Apr-93 & Mar-98 & & $\mathrm{Mn}$ & UOF & Edinst & 444 & 444 & & & & 7 \\
\hline 768 & NSF & $N-92$ & Apr-94 & afayette & IN & PURD & Edlnst & 25 & & & & & 6 \\
\hline 769 & NSF & p-92 & Feb-97 & & $A Z$ & UOF & Edlnst & 212.5 & 37.5 & 62.5 & 62.5 & 0 & 7 \\
\hline 770 & NSF & $g-92$ & Jan-96 & & NU & PAINC & PriEdlns & 275 & 2.833 & 108.5 & 108.5 & 0 & \\
\hline 771 & NSF & $30-92$ & May-98 & & 11 & UOF II & Edlinst & 275 & 12.5 & & & & 7 \\
\hline 772 & NSF & 992 & $\operatorname{san}-97$ & gae Park & $1 M$ & EGE PARK & Edinst & 212.5 & & 62.5 & 62.5 & 0 & \\
\hline 773 & NSF & p-93 & May-94 & & (NNY & APPL & IndBus & 49.9 & & & & & 6 \\
\hline 774 & NSF & nn-93 & & & $\mathrm{FL}$ & HSA & IndBus & & 48.125 & & & & 7 \\
\hline 775 & NSF & & & & $\mathrm{FL}$ & FLOP & Edinst & & 30 & & & & \\
\hline 776 & NSF & $19-92$ & Nov-94 & & OK & UOF OKLAHOMA & Edinst & 60.926 & 10 & & & & 7 \\
\hline 777 & NSF & & & & NY & POLYTECHNIC UN & PriEdIns & 49.996 & 996 & & & & \\
\hline 778 & NSF & $2 y-93$ & Oat-95 & Columbia & MO & U OF MISSOURI & Edlnst & 150 & 50 & 50 & & 0 & 3 \\
\hline 779 & NSF & & & & II & UOF ILL URBAN & & & 75 & 71.391 & 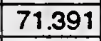 & & \\
\hline 780 & NSF & Sep-93 & Feb-95 & Detroit & $\mid \mathrm{MI}$ & WAYNE STATE. UNIVERSI & Edlnst & 82.247 & 82.247 & & & & \\
\hline
\end{tabular}




\begin{tabular}{|c|c|c|c|c|c|c|}
\hline & Agency & Bureau & $\begin{array}{r}\text { Program } \\
\end{array}$ & Project & Award Number & $\begin{array}{l}\text { Award } \\
\text { Type }\end{array}$ \\
\hline 781 & NSF & ENG & Civil \& mechanical sysiems (CMS) & Large Structural \& Building Systems & 9300745 & $E / G$ \\
\hline 782 & NSF & ENG & Civil \& mechanical systems (CMS) & Structural Systems & 9300827 & E/G \\
\hline 783 & NSF & ENG & Civil \& mochanical systems (CMS) & Nalural \& Man-Made Hazard Mitigation & 9300961 & $E / G$ \\
\hline 784 & NSF & ENG & Civil \& mochanical systems (CMS) & Structural Systems & 9301238 & $E / G$ \\
\hline 785 & NSF & ENG & Civil \& mechanical systems (CMS) & Structural Systems \& Construction Processes & 9301307 & $E / G$ \\
\hline 786 & NSF & ENG & Civil \& mechanical systems (CMS) & Strucural Systems \& Construction Processes & 9301387 & $E / G$ \\
\hline 787 & NSF & ENG & Civil \& mechanical systems (CMS) & Structural Systems & 9301464 & EJG \\
\hline 788 & NSF & ENG & Civil \& mechanical systems (CMS) & Structural Systems & 9301574 & EJG \\
\hline 789 & NSF & ENG & Civil \& mechanical systems (CMS) & Nafural 8 Man-Made Hazard Mitigation & 9301577 & $E / G$ \\
\hline 790 & NSF & ENG & Civil \& mochanical systems (CMS) & Structural Systems \& Construction Processes & 9301580 & $E / G$ \\
\hline 791 & NSF & ENG & Civil \& mechanical systems (CMS) & Struclural Sysiems & 9301584 & $E / G$ \\
\hline 792 & NSF & $E N G$ & Civil \& mochanical sysiems (CMS) & Structural Systems \& Construction Processes & 9301674 & $E / G$ \\
\hline 793 & NSF & ENG & Civil \& mechanical systems (CMS) & Structural Systems & 9301778 & $E / G$ \\
\hline 794 & NSF & ENG & Civil \& mechanical systems (CMS) & Large Structural \& Building Systems & 9301829 & $\overline{E / G}$ \\
\hline 795 & NSF & ENG & Civil \& mechanical sysiems (CMS) & Structural Systems & 9301962 & $E / G$ \\
\hline 796 & NSF & ENG & Civil \& mechanical systems (CMS) & Dynamic Systems \& Control & 9301975 & $E / G$ \\
\hline 797 & NSF & ENG & Civil \& mechanical systems (CMS) & Structural Systems & 9301989 & $E / G$ \\
\hline 798 & NSF & ENG & Civil \& mechanical systems (CMS) & Architectural \& Mechanical Systems & 9302101 & $E / G$ \\
\hline 799 & NSF & ENG & Civil \& mechanical systems (CMS) & Structural Systems & 9302102 & $E / G$ \\
\hline 800 & NSF & ENG & Civil \& mechanical systems (CMS) & Architectural \& Mechanical Systems & 9302201 & $E / G$ \\
\hline 801 & NSF & ENG & Civil \& mechanical systems (CMS) & Strucural Systems \& Construction Processes & 9302222 & $E / G$ \\
\hline 802 & NSF & ENG & Civil \& mechanical systems (CMS) & Large Siruciural \& Building Systems & 9302597 & $E / G$ \\
\hline 803 & NSF & ENG & Civil \& mechanical systems (CMS) & Earthquake Systems Integration & 9302612 & $E / G$ \\
\hline 804 & NSF & MPS & Mathematical sciences (DMS) & Applied Mathematics & 9302726 & $E / G$ \\
\hline 805 & NSF & ENG & Electrical \& communications systems (ECS & Systems Theory & 9302926 & $E / G$ \\
\hline 806 & NSF & ENG & Civil \& mechanical systems (CMS) & Large Structural \& Building Systems & 9303138 & $E / G$ \\
\hline 807 & NSF & ENG & Civil \& mechanical systems (CMS) & Architectural \& Mechanical Systems & 9303419 & $E / G$ \\
\hline 808 & NSF & GEO & Earth sciences (EAP) & Geophysias & 9304110 & $E / G$ \\
\hline 809 & NSF & ENG & Civil \& mechanical systerns (CMS) & Structural Systems \& Construction Processes & 9305397 & $E / G$ \\
\hline 810 & NSF & MPS & Mathermatical sciences (DMS) & Infratructure & 9305770 & $E / G$ \\
\hline 811 & NSF & ENG & Civil \& mochanical systems (CMS) & Archilectural \& Mechanical Systems & 9307214 & $E / G$ \\
\hline 812 & NSF & ENG & Civil \& mechanical systems (CMS) & Architectural \& Mechanical Systems & 9307666 & $E / G$ \\
\hline 813 & NSF & ENG & Civil \& mechanical systems (CMS) & Architectural \& Mechanical Systems & 9307822 & $E / G$ \\
\hline 814 & NSF & ENG & Civil \& mechanical systems (CMS) & Architectural \& Mechanical Systoms & 9307840 & $E / G$ \\
\hline 815 & NSF & ENG & Civil \& mechanical systems (CMS) & Architectural \& Mechanical Systems & 9307843 & $E / G$ \\
\hline 816 & NSF & ENG & Civil \& mechanical systems (CMS) & Structural Systems & 9307666 & $E / G$ \\
\hline 817 & NSF & ENG & Civil \& mechanical systems (CMS) & Architectural \& Mechanical Systems & 9307885 & $E / G$ \\
\hline 818 & NSF & ENG & Civil \& mechanical systems (CMS) & Structural Systems \& Construction Processes & 9308188 & $E / G$ \\
\hline 819 & NSF & CSE & Information, robotios \& intelligent systems & Knowledge Models \& Cognitive Systems & 9308308 & $E / G$ \\
\hline 820 & NSF & ENG & Civil \& mechanical systems (CMS) & Architeclural \& Mechanical Systems & 9309149 & $E / G$ \\
\hline 821 & NSF & ENG & Chemical \& transport systems (CTS) & Intertacial, Transpon \& Thermodynamic Process & 9309528 & $E / G$ \\
\hline 822 & NSF & ENG & Civil \& mechanical systems (CMS) & Structural Systems \& Construction Processes & 9309623 & $E / G$ \\
\hline 823 & NSF & ENG & Civil \& mechanicai sysiems (CMS) & Strucural Systems \& Construction Processes & 9309655 & $E / G$ \\
\hline 824 & NSF & ENG & Civil \& mechanical systems (CMS) & Structural Systems \& Construction Processes & 9309754 & $E / G$ \\
\hline 825 & NSF & ENG & Civil \& mechanical systems (CMS) & Structural Systems & 9309757 & $E / G$ \\
\hline 826 & NSF & ENG & Civil \& mechanical systems (CMS) & Structural Systems \& Construction Processes & 9309775 & $E / G$ \\
\hline 827 & NSF & ENG & Dosign, manufacture \& industrial innovation & Computer Integrated Engineering & 9309925 & $E / G$ \\
\hline 828 & NSF & ENG & Civil \& mechanical systems (CMS) & Earthquake Systems Integration & 9310055 & $E / G$ \\
\hline 829 & NSF & ENG & Civil \& mechanical systems (CMS) & Architectural \& Mechanical Systems & 9310169 & $E / G$ \\
\hline 830 & NSF & ENG & Civil \& mechanical systems (CMS) & Structural Systems & 9310490 & $E / G$ \\
\hline 831 & NSF & ENG & Civil \& mechanical systems (CMS) & Structural Systems \& Construction Processes & 9310491 & $E / G$ \\
\hline 832 & NSF & ENG & Civil \& mechanical systems (CMS) & Earthquake Systems Integration & 9310716 & $E / G$ \\
\hline 833 & NSF & ENG & Bioengineering \& environmental systems (B & Environmental Engineering Systems & 9311144 & $E / G$ \\
\hline 834 & NSF & SBE & International cooperative scientific activities & hodia & 9311381 & $E / G$ \\
\hline 835 & NSF & ENG & Civil \& mechanical systems (CMS) & Natural \& Man-Made Hazard Minigation & 9311549 & $E / G$ \\
\hline 836 & NSF & ENG & Civil \& mochanical systems (CMS) & Earthquake Systems Inegration & 9311857 & $E / G$ \\
\hline 837 & NSF & ENG & Civil \& mechanical systems (CMS) & Large Sinutural \& Building Systems & 9311996 & $E / G$ \\
\hline 836 & NSF & ENG & Civil \& mechanical systems (CANS) & Structural Systems \& Construction Processes & 9312281 & $E / G$ \\
\hline 839 & NSF & ENG & Civil \& mechanical systems (CMS) & Geomechanical/Geotech \& Geo-Environmental & 9312418 & $E / G$ \\
\hline 840 & NSF & ENG & Civil \& mechanical systems (CMS) & Archilectural \& Mechanical Systems & 9312640 & $E G$ \\
\hline 841 & NSF & ENG & Civil \& mechanical systems (CMS) & Siting \& Geotechnical Systems & 9312682 & $E / G$ \\
\hline 842 & NSF & ENG & Electrical \& communications systems (ECS & Lightwave Technology & 9312726 & $E / G$ \\
\hline 843 & NSF & ENG & Civil \& mechanical systems (CMS) & Structural Systems & 9312780 & $E / G$ \\
\hline 844 & NSF & ENG & Civil \& mechanical systems (CMS) & Structural Systems & 9312785 & $E / G$ \\
\hline 845 & NSF & ENG & Civil \& mechanical systerms (CMS) & Large Structural \& Building Systems & 9312851 & $E / G$ \\
\hline
\end{tabular}




\begin{tabular}{|c|c|c|c|c|c|c|c|c|c|c|c|c|c|}
\hline & Agency & $\begin{array}{l}\text { Start } \\
\text { Date }\end{array}$ & $\begin{array}{l}\text { End } \\
\text { Date }\end{array}$ & $\begin{array}{c}\text { Place of } \\
\text { Performance }\end{array}$ & $\begin{array}{l}\text { : } \\
\text { जั } \\
\frac{0}{0}\end{array}$ & Performer Name & $\begin{array}{l}\text { Perf } \\
\text { Type }\end{array}$ & $\begin{array}{c}\text { Total } \\
\text { Award } \\
\text { Amount }\end{array}$ & $\begin{array}{l}\text { Average } \\
\text { Annual } \\
\text { Funding }\end{array}$ & $\begin{array}{l}\text { FY } \\
\text { Total } \\
\text { Amt }\end{array}$ & $\begin{array}{c}\text { FY Fed } \\
\text { Amt }\end{array}$ & \begin{tabular}{|l|} 
FY \\
Non- \\
Fed \\
Amt
\end{tabular} & Prod. \\
\hline 781 & NSF & Dec-92 & Nov-94 & Fon Collins & co & COLORADO STATE UNIVE & Edinst & 39.927 & 39.927 & & & & 6 \\
\hline 782 & NSF & Aug-93 & Jan-97 & Notre Dame & IN & UNIVERSITY OF NOTRE D. & PriEdlnst & 170 & 70 & 70 & 70 & 0 & 7 \\
\hline 783 & NSF & Nov-92 & Oct-94 & Honolulu & HI & U OF HAWAII MANOA & Edinst & 42.703 & 42.703 & & & & 7 \\
\hline 784 & NSF & $D_{\theta c-92}$ & Nov-93 & Amherst & NY & SUNY BUFFALO & Edlnst & 20 & 20 & & & & 7 \\
\hline 785 & NSF & Apr-94 & Mar-95 & Berkeley & CA & U OF CAL BERKELEY & Edlnst & 127.545 & 62.428 & 62.428 & 62.428 & 0 & 6 \\
\hline 786 & NSF & Dec-93 & Nov-95 & Ann Arbor & MI & U OF MICHIGAN ANN ARB & Edlnst & 80 & 80 & 80 & 80 & 0 & 7 \\
\hline 787 & NSF & Aug-93 & Jan-96 & Corvallis & OR & OREGON STATE UNIVERS & Edlns & 204.9 & 100 & 102.6 & 102.6 & 0 & 7 \\
\hline 788 & NSF & Jut-93 & Dec-94 & Blacksburg & $\mathrm{VA}$ & VA POLYTECHNIC INST \& & Edlnst & 199.695 & 99.848 & 97.446 & \begin{tabular}{|l|}
97.446 \\
\end{tabular} & $\underline{0}$ & 7 \\
\hline 789 & NSF & Oat-93 & Mar-96 & Seattle & WA & U OF WASHINGTON & Edlnst & 158.121 & 68.908 & 72.816 & \begin{tabular}{|l|}
72.816 \\
\end{tabular} & 0 & 7 \\
\hline 790 & NSF & May-94 & Sep-96 & Lowa City & IA & UNIVERSITY OF IOWA & Edlnst & 182.812 & 59.675 & 59.675 & 59.675 & 0 & 7 \\
\hline 791 & NSF & Sep-93 & Feb-95 & Notre Dame & IN & UNIVERSITY OF NOTRE D & PriEdlnst & 159.999 & 58.783 & 87.567 & 87.567 & 0 & 7 \\
\hline 792 & NSF & May-94 & Oct-95 & Palo Aloo & $\overline{C A}$ & STANFORD UNIVERSITY & PriEdlnst & 138.72 & 64.432 & 64.432 & 64.432 & 0 & 6 \\
\hline 793 & NSF & Jul-93 & Dec-95 & Lafayette & IN & PURDUE UNIV RESEARCH & Edlnst & 135.15 & 44.505 & & & & 7 \\
\hline 794 & NSF & Sep-93 & Feb-96 & Rapid City & $S D$ & SD SCH OF MINES AND TE & Edlnst & 355.044 & 101.362 & 167.72 & 167.72 & 0 & 7 \\
\hline 795 & NSF & Aug-93 & Jul-95 & Berkeley & CA & U OF CAL BERKELEY & Edlnst & 96 & 96 & & & & 7 \\
\hline 796 & NSF & Jul-93 & Doc-94 & Cookeville & TN & TENNESSEE TECHNOLOG & Edlnst & 100 & 50 & 52.165 & 52.165 & 0 & 7 \\
\hline 797 & NSF & Aug-93 & Jul-96 & Berkeley & CA & U OF CAL BERKELEY & Edlnst & 90.11 & 62.555 & 35 & 35 & 0 & 7 \\
\hline 798 & NSF & Aug-93 & Jul-95 & Berkeley & $\overline{C A}$ & UOF CAL BERKELEY & Edlnst & 74.89 & 74.89 & & & & 7 \\
\hline 799 & NSF & Aug-93 & Jul-95 & Invine & CA & UOF CAL IRVINE & Edlnst & 199.999 & 66.5 & 67 & 67 & 0 & 7 \\
\hline 800 & NSF & Aug-93 & $\operatorname{Jan}-95$ & Rolla & MO & U OF MISSOURI ROLLA & Edinst & 245.76 & 122.88 & 122.46 & 122.46 & 0 & 7 \\
\hline 801 & NSF & Mar-94 & Feb-96 & Austin & $T X$ & U OF TEXAS AUSTIN & Edlnst & 211.1 & 71.1 & 71.1 & 71.1 & 0 & 7 \\
\hline 802 & NSF & Apr-93 & Aug-94 & Boulder & $\infty$ & U OF COLORADO BOULDE & Edlnst & 42.916 & 42.916 & & & & 7 \\
\hline 803 & NSF & Jul-93 & Jun-95 & Oakland & CA & ASSN OF BAY AREA GOV & StalocGov & 170 & 102 & 34 & 34 & 0 & 7 \\
\hline 804 & NSF & Jul-93 & Nov-95 & College Park & MD & U OF MD COLLEGE PARK & EdInst & 165.001 & 63 & 71 & 71 & & 8 \\
\hline 805 & NSF & Aug-93 & Jul-95 & Berkeley & CA & U OF CAL BERKELEY & Edlnst & 165 & 55 & 55 & 55 & 0 & 7 \\
\hline 806 & NSF & Sep-93 & Aug-95 & Tueson & $A \bar{Z}$ & UNIVERSITY OF ARIZONA & Edlnst & 24.429 & 18.262 & 12.095 & 12.095 & 0 & 7 \\
\hline 807. & NSF & Mar-93 & Feb-94 & San Diego & $\overline{C A}$ & U OF CAL SAN DIEGO & Edlnst & 50 & 50 & & & & 7 \\
\hline 808 & NSF & Sep-93 & Aug- 94 & Pasadena & $\mathrm{CA}$ & CALIFORNIA INST OF TEC & PriEdinst & 33.707 & 36.047 & 38.387 & 38.387 & 0 & 7 \\
\hline 809 & NSF & Apr -93 & Mar-94 & Washington & $D C$ & CERF & NonprofiNonE & 75 & 75 & & & & 7 \\
\hline 810 & NSF & Aug-93 & Jan-95 & Clemson & SC & CLEMSON UNIVERSITY & Edlnst & 17.973 & 17.973 & & & & 7 \\
\hline 811 & NSF & Apr-93 & Feb-94 & Boca Raton & $\mathrm{FL}$ & FLORIDA ATLANTIC UNIV & Edlnst & 4.7 & 4.7 & & & & 7 \\
\hline 812 & NSF & Aug-93 & Jan-95 & Urbana & IIL & U OF ILL URBANA-CHAMP & Edlnst & 180 & 90 & 81.245 & 81.245 & 0 & 7 \\
\hline 813 & NSF & Sep-93 & Aug-95 & San Diego & $\mathrm{CA}$ & U OF CAL SAN DIEGO & Edlnst & 102 & 36 & 32 & 32 & & 7 \\
\hline 814 & NSF & Sep-93 & Aug-95 & San Diego & $C A$ & U OF CAL SAN DIEGO & Edlnst & 150 & 50 & 50 & 50 & $\underline{0}$ & 7 \\
\hline 815 & NSF & Sep-93 & Jul-95 & Ann Arbor & MI & UNIVERSITY OF MICHIGAI & Edlnst & 240.046 & 80.028 & 79.838 & \begin{tabular}{|l|}
79.838 \\
\end{tabular} & 0 & 7 \\
\hline 816 & NSF & Aug-93 & $\operatorname{Jan}-97$ & Bethlehem & PA & LEHIGH UNIVERSITY & PriEdlnst & 177.641 & 74.436 & 73.754 & 73.754 & 0 & 7 \\
\hline 817 & NSF & Aug-93 & Jan-95 & Urbana & IL & U OF ILL URBANA-CHAMP & Edlnst & 120 & 43.502 & 44.097 & 44.097 & 0 & 7 \\
\hline 818 & NSF & Apr -93 & Sep-94 & Washington & $D C$ & NAT ACAD OF SCIENCES & NonprofiNonE & 25 & 25 & 25 & 25 & 0 & 4 \\
\hline 819 & NSF & Jun-93 & Nov-96 & Arlington & $T X$ & U OF TEXAS ARLINGTON & Edlnst & 162.982 & 99.926 & & & & 8 \\
\hline 820 & NSF & Aug-93 & Jul-96 & College Station & $T X$ & TEXAS ENGINEERING EXP & Edlnst & 89.837 & 44.919 & 19.837 & 19.837 & 0 & 7 \\
\hline 821 & NSF & Oct-94 & Sep-95 & State College & $\overline{P A}$ & PA ST U UNIVE & Edlnst & 170 & 85 & 85 & 85 & 0 & 7 \\
\hline 822 & NSF & May-94 & Sep-96 & Auburn & $A L$ & AUBURN UNIVERSITY & Edlnst & 0 & 0 & & 0 & 0 & 8 \\
\hline 823 & NSF & May-94 & Sep-96 & Palo Alto & $\mathrm{CA}$ & STANFORD UNIVERSITY & PriEdlnst & 100 & 40 & 40 & 40 & $\overline{0}$ & 6 \\
\hline 824 & NSF & Aug-93 & Jul-95 & Minneapolis & MN & U OF MINNESOTA-TWIN C & Edinst & 18 & 18 & & & & 7 \\
\hline 825 & NSF & Aug-93 & $\tan -95$ & Pultman & WA & WASHINGTON STATE UNI & Edlnst & 90 & 31.524 & $5 \overline{1.73}$ & 51.73 & 0 & 7 \\
\hline 826 & NSF & Feb-94 & Jan-96 & Santa Barbara & CA & U OF CAL SANTA BARBAR & Edlnst & 239.716 & 18.224 & 18.224 & 18.224 & 0 & 7 \\
\hline 827 & NSF & Aug-93 & Jul-95 & Berkeley & CA & U OF CAL BERKELEY & Edinst & 25 & 25 & & & & 3 \\
\hline 828 & NSF & $\operatorname{sep}-93$ & Feb-95 & & GA & U OF GEORGIA RES FDNI & Edlnst & 49.064 & 49.064 & & & & 7 \\
\hline 829 & NSF & Jul-93 & Dec-94 & Princeton & NJ & INTER ASSOC STRUC SAF & NonprofiNonE & 15 & 15 & & & & 7 \\
\hline 830 & NSF & Aug-93 & Nov-97 & Richmond & $C A$ & CUREE & NonprofiNonE & 258.494 & 64 & 69.5 & 69.5 & 0 & 7 \\
\hline 831 & NSF & Sep-93 & Aug-94 & New York & NY & COLUMBIA UNIVERSITY & \begin{tabular}{|l|} 
PriEdlnst \\
\end{tabular} & 40.895 & 21.175 & 1.455 & 1.455 & 0 & 7 \\
\hline 832 & NSF & Nov-93 & Apr-96 & Menlo Park & $C A$ & WILLIAM SPANGLE \& ASS & IndBus & 152.017 & 182.394 & 182.39 & 182.39 & 0 & 6 \\
\hline 833 & NSF & Sep-93 & Aug-94 & Riverside & $\mathrm{CA}$ & U OF CAL RIVERSIDE & Edlnst & 195.827 & 97.914 & 95.269 & 95.269 & 0 & 7 \\
\hline 834 & NSF & Jun-93 & May-94 & Boca Raton & $F L$ & FLORIDA ATLANTIC UNIV & Edlnst & 0 & 539.9 & & & & 7 \\
\hline 835 & NSF & Jun-93 & Aug-94 & Los Angeles & $C A$ & U OF CAL LOS ANGELES & Edlnst & 29.501 & 29.501 & & & & 7 \\
\hline 836 & NSF & Jan-94 & Jun-95 & New Orleans & LA & UNIVERSITY OF NEW ORL & PriEdinst & 270.346 & 124.257 & 124.26 & 124.26 & 0 & 2 \\
\hline 837 & NSF & May-93 & Oct-94 & Boulder & CO & ATKINSON-NOLAND ASSO & IndBus & 8.298 & 8.298 & & & & 7 \\
\hline 838 & NSF & Apr-93 & Mar-95 & Philadelphia & PA & DREXEL UNIVERSITY & Priedlnst & 48.847 & 48.847 & & & & 7 \\
\hline 839 & NSF & Jun-93 & May-94 & Cambridge & MA & & PriEdinst & 17.464 & 17.464 & & & & 7 \\
\hline & NSF & & Jul-95 & Boca Baton & $F L$ & FLORIDA ATLANTKC UNIV & Edins & 149.988 & 149.988 & & & & 7 \\
\hline 841 & NSF & May-93 & OCt-94 & Albuquerque & NM & APPLIED RESEARCH ASS & IndBus & 48.918 & 48.918 & & & & 7 \\
\hline 842 & NSF & Aug-93 & Jan-95 & New Brunswick & CNN & RUTGERS U NEW BRUNS & Edlnst & 50 & 50 & & & & 7 \\
\hline 843 & NSF & May-93 & OCt-94 & Princeton & NU & PRINCETON UNIVERSITY & PriEdlnst & 21.82 & 21.82 & & & & 7 \\
\hline 844 & NSF & May-93 & Apr-94 & Buffalo & $\mathrm{NY}$ & SUNY BUFFALO & Edlnst & 35.112 & 35.112 & & & & 7 \\
\hline 845 & NSF & Sep-94 & Jul-97 & Reno & NV & U OF NEVADA RENO & Edlnst & 166.512 & 16.253 & 16.253 & 16.253 & & 7 \\
\hline
\end{tabular}




\begin{tabular}{|c|c|c|c|c|c|c|}
\hline & Ageney & Bureau & Program & Proloct & Award Number & Award \\
\hline 846 & NSF & ENG & Civil \& mochanical systems (CMS) & Structural Systems \& Construction Processes & 9312954 & $E / G$ \\
\hline 847 & NSF & ENG & Civil \& mechanical systems (CMS) & Large Structural \& Building Systems & 9313062 & $E / G$ \\
\hline 848 & NSF & ENG & Civil \& mechanical systems (CMS) & Siructural Systems \& Construction Processes & 9313076 & $E / G$ \\
\hline 849 & NSF & ENG & Civil \& mochanical systems (CMS) & Structural Systems \& Construction Processes & 9313122 & $E / G$ \\
\hline 850 & NSF & ENG & Design, manufacture $\&$ industrial innovation & Engineering Design & 9313186 & $E / G$ \\
\hline 851 & NSF & ENG & Bioengineering \& environmental systems (A & Environmental Engineering Systems & 9313766 & $E / G$ \\
\hline 852 & NSF & ENG & Electrical \& communications systems (ECS & Neuroenginesing & 9313934 & E/G \\
\hline 853 & NSF & ENG & Civil \& mechanical systerms (CMS) & Structural Systems & 9314197 & E/G \\
\hline 854 & NSF & ENG & Civil \& mechanical systems (CMS) & Structural Systems & 9314435 & $E / G$ \\
\hline 855 & NSF & ENG & Civil \& mechanical systems (CMS) & Strucural Systems \& Construction Processes & 9314527 & $E / G$ \\
\hline 856 & NSF & ENG & Civil \& mechanical systems (CMS) & Structural Systems & 9314767 & E/G \\
\hline 857 & NSF & SBE & International cooperative scientific activities & Japan & 9314853 & $E / G$ \\
\hline 858 & NSF & SBE & International cooperative scientific activities & Japan & 9315122 & $E / G$ \\
\hline 859 & NSF & ENG & Civil \& mechanical systems (CMS) & Architectural \& Mechanical Systems & 9315240 & $E / G$ \\
\hline 860 & NSF & ENG & Engineering education \& conters (EEC) & Engineering Education & 9315602 & $E / G$ \\
\hline 861 & NSF & SBE & International cooperative scientific activities & Japan & 9315649 & $E / G$ \\
\hline 862 & NSF & ENG & Civil \& mechanical systems (CMS) & Siting \& Geotechnical Systems & 9315694 & $E / G$ \\
\hline 863 & NSF & ENG & Civil \& mechanical systems (CMS) & Natural \& Man-Made Hazard Mitigation & 9316983 & $E / G$ \\
\hline 864 & NSF & ENG & Civil \& mechanical systems (CMS) & Earthquake Systems Integration & 9317461 & $E / G$ \\
\hline 865 & NSF & ENG & Civil \& mechanical systems (CMS) & Structural Syslems & 9317722 & $E / G$ \\
\hline 866 & NSF & ENG & Civil \& mechanical systems (CMS) & STructural Systems \& Construction Processes & 9317851 & $E / G$ \\
\hline 867 & NSF & ENG & Civil \& mechanical systems (CMS) & Natural \& Man-Made Hazard Mitigation & 9317876 & $E / G$ \\
\hline 868 & NSF & $\mathrm{BIO}$ & Biological instrumentation \& resources (BIR & Instrumentation \& Instrument Development & 9317890 & $E / G$ \\
\hline 869 & NSF & MPS & Physics (PHY) & Theoretical Physics & 9318152 & $E / G$ \\
\hline 870 & NSF & ENG & Civil \& mechanical systems (CMiS) & Siting \& Geotechnical Systems & 9318163 & $E / G$ \\
\hline 871 & NSF & MPS & Mathematical sciences (DMS) & Computational Mathematics & 9318166 & $E / G$ \\
\hline 872 & NSF & $B 10$ & Biological instrumentation \& resources (BIR & Databases, Software Development \& Comp & 9318169 & $E / G$ \\
\hline 873 & NSF & $\mathrm{BIO}$ & Biological instrumentation \& resources (BIR & Databases, Software Development \& Comp & 9318183 & $E / \bar{G}$ \\
\hline 874 & NSF & MPS & Mathematical sciences (DMS) & Computational Mathematics & 9318184 & $E / G$ \\
\hline 875 & NSF & MPS & Astronomical sciences (AST) & Extragalactic Astronomy \& Cosmology & 9318185 & $E / G$ \\
\hline 876 & NSF & SBE & International cooperative scientific activities & Americas & 9318323 & $E / G$ \\
\hline 877 & NSF & ENG & Civil \& mechanical systems (CMS) & Architectural \& Mechanical Systems & 9318776 & $E / G$ \\
\hline 878 & NSF & ENG & Civil \& mechanical systems (CMS) & Architectural \& Mechanical Systems & 9318997 & $E / G$ \\
\hline 878 & NSF & ENG & Civil \& mechanical systems (CMS) & Large Structural \& Building Systems & 9319204 & $E / G$ \\
\hline 880 & NSF & ENG & Civil \& mechanical systems (CMS) & Structural Systems & 9319341 & $E / G$ \\
\hline 881 & NSF & ENG & Civil \& mechanical systems (CMS) & Architectural \& Mechanical Systems & 9319434 & $E / G$ \\
\hline 882 & NSF & MPS & Chemistry (CHE) & Statistical \& Simulations & 9319519 & $E / G$ \\
\hline 883 & NSF & ENG & Civil \& mechanical systems (CMS) & Large Structural \& Building Systems & 9319802 & $E / G$ \\
\hline 884 & NSF & ENG & Civil \& mechanical systems (CMS) & Structural Systems & 9319838 & $E / G$ \\
\hline 885 & NSF & ENG & Civil \& mechanical systems (CMS) & Nalural \& Man-Made Hazard Mitigation & 9319841 & $E / G$ \\
\hline 886 & NSF & ENG & Civil \& mechanical systems (CAS) & STructural Systems \& Construction Procosses & 9319878 & E/G \\
\hline 887 & NSF & ENG & Civil \& mechanical systems (CMS) & Structural Systems & 9320014 & $E / G$ \\
\hline 888 & NSF & ENG & Engineering education \& centers (EEC) & Industry/University Cooperative Research Cente & 9320137 & $E / G$ \\
\hline 889 & NSF & ENG & Civil \& mechanical systems (CMS) & Archilectural \& Mechanical Systems & 9320150 & $E / G$ \\
\hline 890 & NSF & SBE & International cooperative scientific activities & Western Europe & 9320409 & $E / G$ \\
\hline 891 & NSF & ENG & Electrical \& communications systems (ECS & Quantum Electronics, Plasma \& Electromechar & 9320515 & $E / G$ \\
\hline 892 & NSF & ENG & Civil \& mechanical systems (CMS) & Structural Systems \& Consiruction Processes & 9320896 & $E / G$ \\
\hline 893 & NSF & ENG & Civil \& mechanical systems (CMS) & Sructural Systems \& Construction Procosses & 9321346 & $E / G$ \\
\hline 894 & NSF & ENG & Chemical \& transport systems (CTS) & Process \& Reaction Engineering & 9321863 & $E / G$ \\
\hline 895 & NSF & ENG & Civil \& mechanical systems (CMS) & Natural \& Man-Made Hazard Mitigation & 9322507 & $E / G$ \\
\hline 896 & NSF & ENG & Civil \& mechanical systems (CMS) & Archilectural \& Mechanical Systems & 9322524 & $E / G$ \\
\hline 897 & NSF & ENG & Civil \& mechanical systems (CMS) & Large Structural \& Building Systems & 9322607 & $E / G$ \\
\hline 898 & NSF & ENG & Civil \& mechanical systems (CMS) & Natural \& Man-Made Hazard Mitigation & 9322717 & $E / G$ \\
\hline 899 & NSF & ENG & Civil \& mechanical systems (CMS) & Natural \& Man-Made Hazard Mitigation & 9322720 & $E / G$ \\
\hline 900 & NSF & ENG & Chemical \& transport systems (CTS) & Separation \& Purification Processes & 9322772 & $E / G$ \\
\hline 901 & NSF & EHR & \multicolumn{2}{|c|}{ Graduate education \& research development (GER) } & 9350076 & $E / G$ \\
\hline 902 & NSF & EHR & Graduate education \& research developmen & nt (GER) & 9355029 & $E / G$ \\
\hline 903 & NSF & CSE & Computer \& computation research (CCR) & Computer Systems Architecture & 9357790 & E/G \\
\hline 904 & NSF & ENG & Civil \& mechanical systems (CMS) & Large Structural \& Building Systems & 9358075 & $E / G$ \\
\hline 905 & NSF & ENG & Civil \& mechanical systems (CMS) & Sinuctural Systems & 9358186 & $E / G$ \\
\hline 906 & NSF & ENG & Design, manufacture $\&$ industrial innovation & SBIR-Phase I & 9360007 & E/G \\
\hline 907 & NSF & ENG & Design, manufacture \& industrial innovation & SBIR-Phase I & 9360055 & $E / G$ \\
\hline 908 & NSF & ENG & Design, manufacture $\&$ industrial innovation & SBIR-Phase I & 9360497 & $E / G$ \\
\hline 909 & NSF & ENG & Design. manufacture $\&$ industrial innovation & SBIR-Phase I & 9360798 & $E / G$ \\
\hline 910 & NSF & ENG & Design. manufacture \& industrial innovation & SBIR-Phase I & 9361511 & $E / G$ \\
\hline
\end{tabular}




\begin{tabular}{|c|c|c|c|c|c|c|c|c|c|c|c|c|c|}
\hline & Agency & $\begin{array}{l}\text { Start } \\
\text { Date }\end{array}$ & $\begin{array}{l}\text { End } \\
\text { Date }\end{array}$ & $\begin{array}{c}\text { Place of } \\
\text { Performance }\end{array}$ & $\begin{array}{l}0 \\
5 \\
5 \\
0 \\
0\end{array}$ & Performer Name & 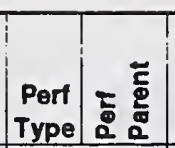 & $\begin{array}{c}\text { Total } \\
\text { Award } \\
\text { Amount }\end{array}$ & $\begin{array}{l}\text { Average } \\
\text { Annual } \\
\text { Funding }\end{array}$ & $\begin{array}{l}\text { FY } \\
\text { Total } \\
\text { Amt }\end{array}$ & $\begin{array}{c}\text { FY Fod } \\
\text { Amt }\end{array}$ & $\begin{array}{c}\text { Fy } \\
\text { Non- } \\
\text { Fed } \\
\text { Amt }\end{array}$ & $\begin{array}{c}\text { Prod. } \\
\end{array}$ \\
\hline 846 & NSF & May-94 & Sep-96 & Palo Alto & $C A$ & STANFORD UNIVERSITY & PriEdinst & 220 & 70.168 & 70.168 & 70.168 & 0 & \begin{tabular}{|l|l|}
8 \\
\end{tabular} \\
\hline 847 & NSF & Jan-94 & \begin{tabular}{|l|} 
Jun-96 \\
\end{tabular} & Cambridge & MA & MIT & PriEdinst & 246.655 & 80.424 & 80.424 & \begin{tabular}{|l|}
80.424 \\
\end{tabular} & 0 & $\frac{7}{7}$ \\
\hline 848 & NSF & Jun-94 & \begin{tabular}{|l|} 
Oct-96 \\
\end{tabular} & Lafayelle & IN & PURDUE UNIV RESEARCH & Edinst & 210 & 65.29 & 65.29 & 65.29 & 0 & 7 \\
\hline 849 & NSF & May-94 & Oct-95 & Evanston & $\sqrt{\mathrm{IL}}$ & NORTHWESTERN UNIVEA & PriEdlinst & 14.996 & 14.996 & 14.996 & 14.996 & 0 & 7 \\
\hline 850 & NSF & Sep-93 & Feb-96 & Boulder & $\infty$ & U OF COLORADO BOULDA & Edlnst & 140 & 70 & 70 & 70 & 0 & 8 \\
\hline 851 & NSF & Sep-93 & Aug-94 & Ann Arbor & MI & U OF MICHIGAN ANN ARB & Edlnst & 7.019 & 7.019 & & & & 7 \\
\hline 852 & NSF & Aug-93 & Jun-95 & Battimore & MD & JOHNS HOPKINS UNIVER & SPriEdlnst & 220.554 & 72.399 & 71.248 & 71.248 & 0 & 7 \\
\hline 853 & NSF & Sep-93 & Feb-95 & Uribana & II & U OF ILL URBANA-CHAMP & Edlnst & 47.2 & 23.6 & 0 & 0 & 0 & 7 \\
\hline 854 & NSF & Jun-93 & Nov-93 & Washinglon & $D C$ & TRAVEL AWARD & Privindiv & 0 & 3.569 & & & & 7 \\
\hline 855 & NSF & Jun-94! & Mar-96 & Austin & $T \bar{X}$ & UOF TEXAS AUSTIN & Edlnst & 200 & 67.673 & 67.673 & 67.673 & 0 & 8 \\
\hline 856 & NSF & May-93 & Feb-94 & Washington & $D C$ & TRAVEL AWARD & Privindiv & 1.74 & 1.74 & & & & 7 \\
\hline 857 & NSF & Apr-94 & Sep-96 & Richmond & $\mathrm{CA}$ & CUREE & NonproNionE & 28.25 & 28.25 & 28.25 & 28.25 & 0 & 7 \\
\hline 858 & NSF & Apr-94 & Sep-95 & Minneapolis & MN & UOF MINNESOTA-TWIN C & EdInst & 15 & 15 & 15 & 15 & 0 & 7 \\
\hline 859 & NSF & Jun-94 & ad-95 & Uibana & ill & U OF ILL URBANA-CHAMP & Edlnst & 135.094 & 69.752 & 69.752 & 69.752 & 0 & 7 \\
\hline 860 & NSF & Jul-94 & Dec-95 & Lubbock & $T x$ & TEXAS TECH UNIVERSITY & Edlnst & 89.998 & 89.998 & 89.998 & 89.998 & 0 & 7 \\
\hline 861 & NSF & Apr-94 & Mar-96 & thaca & NY & CORNELL UNIVERSITY-EN & PriEdlnst & 8.75 & 8.75 & 8.75 & 8.75 & 0 & 7 \\
\hline 862 & NSF & Aug-93 & Jan-96 & Brooklyn & NY & POLYTECHNIC UNIVERSIT & PriEdinst & 137.498 & 30.552 & 42.173 & 42.173 & 0 & 7 \\
\hline 863 & NSF & Sep-93 & Feb-96 & Washington & $D C$ & NAT ACAD OF SCIENCES & NonprofNanE & 85 & 37.5 & 10 & 10 & 0 & 7 \\
\hline 864 & NSF & Dec-93 & May-95 & Memphis & ITN & CENTRAL U.S. EARTHQUA & NonprofiNonE & 31.5 & 31.5 & 31.5 & 31.5 & 0 & 7 \\
\hline 865 & NSF & Sep-93 & Feb-97 & Palo Alto & $\mathrm{CA}$ & STANFORD UNIVERSITY & \begin{tabular}{|l|} 
PriEdlnst \\
\end{tabular} & 104.85 & 54.921 & 4.992 & 4.992 & 0 & 7 \\
\hline 866 & NSF & May-94 & Oct-96 & Minneapolis & MN & U OF MINNESOTA-TWIN C & Edlnst & 68.756 & 20.366 & 20.366 & 20.366 & 0 & 7 \\
\hline 867 & NSF & May-94 & Oct-97 & Clemson & $S C$ & CLEMSON UNIVERSITY & Edlnst & 335.732 & 104.217 & 104.22 & 104.22 & 0 & 7 \\
\hline 868 & NSF & Feb-94 & Jul-96 & Boulder & co & U OF COLORADO BOULDE & Edlnst & 202.5 & 202.5 & 202.5 & 202.5 & 0 & 7 \\
\hline 869 & NSF & Sep-93 & Aug-98 & Austin & $T X$ & UOF TEXAS AUSTIN & EdInst & 3750 & 568.5 & 887 & 887 & 0 & 7 \\
\hline 870 & NSF & Sep-93 & Feb-98 & Oakland & PA & CARNEGIE MELLON UNIVE & 6 PriEdlnst & 2154 & 537 & 540 & 540 & 0 & 7 \\
\hline 871 & NSF & Sep-93 & Feb-99 & Palo Alio & CA & STANFORD UNIVERSITY & PriEdinst & 3210 & 570 & 665 & 665 & 0 & 6 \\
\hline 872 & NSF & Sep-93 & Feb-97 & Tucson & $A Z$ & U OF ARIZONA & Edlnst & 900 & 405 & 510 & 510 & 0 & $\frac{7}{7}$ \\
\hline 873 & NSF & Sep-93 & Feb-99 & College Park & MD & U OF MD COLLEGE PARK & Edlnst & 2965.7 & 687.5 & 809.3 & 809.3 & 0 & 7 \\
\hline 874 & NSF & Sep-93 & \begin{tabular}{|l|} 
May-95 \\
\end{tabular} & Troy & NY & RENSSELAER POLYTECH & PriEdinst & 2550 & 525 & 500 & 500 & 0 & 7 \\
\hline 875 & NSF & Sep-93 & Feb-99 & Princeton & NJ & PRINCETON UNIVERSITY & PriEdlnst & 3200 & 512.5 & 725 & 725 & & 7 \\
\hline 876 & NSF & Apr-94 & Mar-96 & Austin & $T X$ & UOF TEXAS AUSTIN & Edlnst & 12.43 & 12.43 & 12.43 & 12.43 & & 7 \\
\hline 877 & NSF & Feb-94 & Jul-95 & Moscow & ID & UNIVERSITY OF IDAHO & Edinst & 199.55 & 94.959 & 94.959 & 94.959 & 0 & 7 \\
\hline 876 & NSF & Feb-94 & Jul-95 & Boston & MA & NORTHEAS & PriEdlnst & 180.926 & 100.346 & 100.35 & 100.35 & 0 & 7 \\
\hline 879 & NSF & Sep-94 & Aug-96 & Boulder & co & UOF COLORADO BOULDE & Edinst & 199.42 & 68.479 & 68.479 & 68.479 & 0 & 7 \\
\hline 880 & NSF & Oct-94 & Aug-95 & & PA & UOF PITTSBURGH & PriEdlnst & 180 & 116.742 & 116.74 & 116.74 & 0 & 7 \\
\hline 881 & NSF & May-94 & Sep-95 & Palo Alto & $\mathrm{CA}$ & STANFORD UNIVERSITY & PriEdinst & 195.407 & 92.962 & 92.962 & 92.962 & 0 & $\frac{1}{7}$ \\
\hline 882 & NSF & Feb-94 & Jan-96 & Los Angeles & $\mathrm{CA}$ & U OF CA & Edlnst & 400 & 156 & 156 & 156 & & 7 \\
\hline 883 & NSF & Nov-93 & Apr-95 & State College & PA & PA ST U UNIVERSITY PAR & Edlnst & 50 & 55 & 55 & 55 & & 7 \\
\hline 884 & NSF & Jan-94 & Dec-95 & Cincinnati & $\mathrm{OH}$ & UOF CIN & Edlnst & 155.438 & 90.568 & 90.568 & 90.568 & & 7 \\
\hline 885 & NSF & Sep-94 & Jul-96 & Raleigh & NC & NORTH CAROLINA STATE & Edlnst & 179.248 & 47.7 & 47.7 & 47.7 & & 7 \\
\hline 886 & NSF & Sep-94 & Ausg-96: & Cincinnati & $\mathrm{OH}$ & UOF CIN & Edlnst & 200 & 95.4 & 95.4 & 95.4 & & 7 \\
\hline 887 & NSF & Sep-93 & Feb-95 & Blacksburg & VA & VIRGINIIA POLYTECHNIC II & Edlnst & 10.044 & 10.044 & 10.043 & 10.043 & 0 & $\frac{7}{7}$ \\
\hline 888 & NSF & Sep-93 & Dec-93 & College Station & $7 x$ & TEXAS A\&M ENGINEER E) & Edinst & 10 & 10 & & & & 5 \\
\hline 889 & NSF & Oct-94 & Aug-96 & Lafayette & IN & IE UNIVERSITY & Edinst & 199.833 & 199.833 & 199.83 & 199.83 & 0 & 7 \\
\hline 890 & NSF & Apr-94 & Sep-95 & Rapid City & $S D$ & SD SCH OF MINES AND TE & Edlnst & 18.57 & 18.57 & 18.57 & \begin{tabular}{|l|}
18.57 \\
\end{tabular} & 0 & $\frac{1}{7}$ \\
\hline 891 & NSF & Aug-94 & \begin{tabular}{|l|} 
Jul-97 \\
\end{tabular} & Madison & WI & U OF WISCONSIN MADISC & Edinst & 265.63 & 88.662 & 88.662 & 88.662 & 0 & 7 \\
\hline 892 & NSF & May-94 & Apr-96 & Washington & $D C$ & CERF & NonproinonE & 69.982 & 69.982 & 69.982 & 69.982 & & 7 \\
\hline 893 & NSF & Doc-93 & May-95 & Atlanta & $G A$ & GA TECH RES CORP - GIT & Edinst & 20 & 20 & 20 & 20 & 0 & 8 \\
\hline 894 & NSF & Jul-94 & Jun-95 & Cambridge & MA & MIT & PriEdlinst & 50 & 49.295 & 49.295 & 49.295 & & 8 \\
\hline 895 & NSF & Nov-93 & Jun-95 & Louisville & KY & U OF LOUISVILLE RES FD & Edlnst & 45.566 & 45.566 & 45.566 & 45.566 & & 7 \\
\hline 896 & NSF & Oct-94 & Jut-97 & Pabo allo & $C A$ & STANFORD UNIVERSITY & PriEdlnst & 149.886 & 149.886 & 149.89 & 149.89 & 0 & 7 \\
\hline 897 & NSF & Feb-94 & Jan-95 & Ann Ar & MI & UOFMICHIGAN ANN ARBC & dEdlnst & 38.995 & 38.995 & 38.995 & 38.995 & & 7 \\
\hline 898 & NSF & Dec-93 & Nov-95 & Rolla & MO & U OF MISSOURI ROLLA & Edlnst & 50 & 50 & 50 & 50 & 0 & 5 \\
\hline 899 & NSF & Dec-93 & May-95 & Blacksburg & VA & VIRGINIA POLYTECHNIC II & Edlnst & 49.932 & 49.932 & 49.932 & 49.932 & & 7 \\
\hline 900 & NSF & Feb-94 & Oca-94 & Ausim & $T X$ & CACHE CORPORATION & NonprofNonE & 15 & 15 & 15 & 15 & 0 & 7 \\
\hline 901 & NSF & Sep-93 & Feb-96: & Corvallis & $O R$ & OREGON STATE & Edinst & 186.299 & 186.299 & & & & 7 \\
\hline 902 & NSF & Oct-93 & Mar-97 & TuCs & $A Z$ & UNIVERSITY OF ARIZONA & Edlnst & 597.5 & 239 & & & & 7 \\
\hline 903 & NSF & Aug-93 & Jan-97 & Seanle & WA & U OF WASHINGTON & Edinst & 312.5 & 46.25 & 67.5 & 67.5 & 0 & 8 \\
\hline 904 & NSF & Sep-93 & Fet-97 & Salt Lake City & $U T^{-}$ & UNIVERSITY OF UTAH & Edinst & $\frac{275}{275}$ & 41.856 & 58.712 & 58.712 & 0 & 7 \\
\hline 905 & NSF & Sep-93 & Aug-97: & Austin & TX & U OF TEXAS AUSTIN & Edlnst & 275 & 43.75 & 62.5 & 62.5 & 0 & 7 \\
\hline 906 & NSF & Jan-94 & Dec-94 & Carson City & NV & CARSONITE INTERNATION & IndBus & 62.64 & 62.64 & 62.64 & 62.64 & 0 & 7 \\
\hline 907 & NSF & Jan-94 & Dec-94 & La Place & LA & TECHNOLOGY INTERNATI & IIndBus & 64.656 & 64.656 & 64.656 & 64.656 & 0 & 5 \\
\hline 908 & NSF & Jan-94 & Dec-94 & Attanta & $\overline{G A}$ & STRAIN MONITOR SYSTEN & IndBus & 64.861 & 64.861 & 64.861 & 64.861 & 0 & 7 \\
\hline 909 & NSF & Jan-94 & Dec-94 & Lithonia & GA & EARTHWATER CORPORA & IndBus & 65 & 65 & 65 & 65 & 0 & 7 \\
\hline 910 & NSF & Mar-94 & Jan-95 & Sunnyale & CA & INTELLIGENT MACHINES & IndBus & 64.028 & 64.028 & 64.028 & 64.028 & 0 & 7 \\
\hline
\end{tabular}




\begin{tabular}{|c|c|c|c|c|c|c|c|}
\hline & Agency & Bureau & $\begin{array}{r}\text { Program } \\
\end{array}$ & Project & Award & Number & $\begin{array}{l}\text { Award } \\
\text { Type }\end{array}$ \\
\hline 911 & NSF & ENG & Design, manufacture \& industrial innovation & SBIR-Phase I & 9362052 & & $E / G$ \\
\hline 912 & NSF & ENG & Civil \& mechanical systems (CMS) & Large Structural \& Building Systems & 9400223 & & $E / G$ \\
\hline 913 & $\sqrt{\text { NSF }}$ & ENG & Civil \& mechanical systems (CMS) & Natural \& Man-Made Hazard Mitigation & 9400521 & & $E / G$ \\
\hline 914 & NSF & ENG & Civil \& mechanical systems (CMS) & Structural Systems & 9400744 & & $E / G$ \\
\hline 915 & NSF & ENG & Civil \& mechanical systems (CMS) & Large Structural \& Building Systems & 9400752 & - & $E / G$ \\
\hline 916 & NSF & ENG & Civil \& mechanical systems (CMS) & Large Structural \& Building Systems & 9401211 & . & $E / G$ \\
\hline 917 & NSF & ENG & Civil \& mechanical systems (CMS) & Structural Systems \& Construction Processes & 9402049 & & $E / G$ \\
\hline 918 & NSF & ENG & Civil \& mechanical systems (CMS) & Structural Systems \& Construction Processes & 9402094 & & $E / G$ \\
\hline 919 & NSF & ENG & Civil \& mechanical systems (CMS) & Large Structural \& Building Systems & 9402170 & $\therefore$ & $E / G$ \\
\hline 920 & NSF & ENG & Civil \& mechanical systems (CMS) & Natural \& Man-Made Hazard Mitigation & 9402196 & & $E / G$ \\
\hline 921 & NSF & ENG & Civil \& mechanical systems (CMS) & Large Structural \& Building Systems & 9402224 & 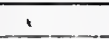 & $E / G$ \\
\hline 922 & NSF & CSE & Computer \& computation research (CCR) & Theory of Computing & 9402334 & & $E / G$ \\
\hline 923 & NSF & ENG & Civil \& mechanical systems (CMS) & Structural Systems \& Construction Procosses & 9402392 & - & $E / G$ \\
\hline 924 & NSF & ENG & Civil \& mechanical systems (CMS) & Structural Systems & 9402535 & & $E / G$ \\
\hline 925 & NSF & ENG & Civil \& mechanical systems (CMS) & Architectural \& Mechanical Systems & 9402572 & & $E / G$ \\
\hline$\overline{926}$ & NSF & ENG & Civil \& mechanical systems (CMS) & Dynamic Systems \& Control & 9402608 & & $E / G$ \\
\hline 927 & INSF & ENG & Civil \& mechanical systems (CMS) & Large Structural \& Building Systems & 9402671 & & $E / G$ \\
\hline 928 & NSF & ENG & Civil \& mechanical systems (CMS) & Architectural \& Mechanical Systems & 9402802 & & $E / G$ \\
\hline 929 & NSF & ENG & Engineering education \& centers (EEC) & Engineering education \& cenlers - Other & 9402989 & & $\mathrm{E} / \mathrm{CA}$ \\
\hline 930 & NSF & ENG & Civil \& mechanical systems (CMS) & Large Structural \& Building Systems & 9403226 & & $E / G$ \\
\hline 931 & NSF & ENG & Civil \& mechanical systems (CMS) & Structural Systems \& Construction Processes & 9403350 & & $E / G$ \\
\hline 932 & NSF & ENG & Civil \& mechanical systems (CMS) & Large Structural \& Building Systems & 9403358 & & $E / G$ \\
\hline 933 & NSF & ENG & Bioengineening \& environmental systems (B) & Environmental Engineering Systems & 9403359 & & $E / G$ \\
\hline 934 & NSF & MPS & Mathematical sciences (DMS) & Computational Mathematics & 9403563 & & $E / G$ \\
\hline$\overline{935}$ & NSF & $E N G$ & Civil \& mechanical systems (CMiS) & Structural Systems & 9403592 & & $E / G$ \\
\hline$\overline{936}$ & NSF & $E N G$ & Civil \& mochanical systems (CMS) & Structural Systems & 9403612 & & $E / G$ \\
\hline 937 & NSF & ENG & Civil \& mechanical systems (CMS) & Architectural \& Mechanical Systems & 9403743 & & $E / G$ \\
\hline 938 & NSF & ENG & Civil \& mechanical systems (CMS) & Earthquake Systems Integration & 9403843 & & $E / \bar{G}$ \\
\hline 939 & NSF & MPS & Mathematical sciences (DMS) & Applied Mathematics & 9403890 & & $E / G$ \\
\hline 940 & NSF & SBE & International cooperative scientific activities & East Asia \& Pacific & 9405343 & & $E / G$ \\
\hline 941 & NSF & ENG & Bioengineering \& environmental systems (B & Environmental Engineering Systems & 9406083 & & $E / G$ \\
\hline 942 & NSF & MPS & Mathematical sciences (DMS) & Applied Mathematics & 9407738 & & $E / G$ \\
\hline 943 & NSF & ENG & Civil \& mechanical systems (CMS) & Structural Systems & 9408162 & & $E / G$ \\
\hline 944 & NSF & ENG & Chemical \& transport systems (CTS) & Fluid Dynamics \& Hydraulies & 9408939 & & E/G \\
\hline 945 & NSF & ENG & Engineering education \& centers (EEC) & Engineering education \& centers - Other & 9409190 & & $E / G$ \\
\hline 946 & NSF & ENG & Civil \& mechanical systems (CMS) & Architectural \& Mechanical Systems & 9409268 & & $E / G$ \\
\hline 947 & NSF & ENG & Civit \& mechanical systems (CMS) & Structural Systems \& Construction Processes & 9409526 & & $E / G$ \\
\hline 948 & NSF & ENG & Civil \& mechanical systerns (CMS) & Large Structural \& Building Systems & 9409870 & & $E / G$ \\
\hline 949 & NSF & ENG & Civil \& mechanical systems (CMS) & Large Strucuural \& Building Systems & 9410091 & & $E / G$ \\
\hline 950 & NSF & ENG & Civil \& mechanical systems (CMS) & Struclural Systems \& Construction Processes & 9410390 & & $E / G$ \\
\hline 951 & NSF & ENG & Civil \& mechanical systems (CMS) & Architectural \& Mechanical Systems & 9410473 & & $E / G$ \\
\hline 952 & NSF & ENG & Civil \& mechanical systems (CMS) & Large Structural \& Building Systems & $9 4 \longdiv { 1 0 6 4 7 }$ & & $E / G$ \\
\hline 953 & $\widehat{N S F}$ & ENG & Civil \& mechanical systems (CMS) & Structural Systems \& Consiruction Processes & 9410683 & & $E / G$ \\
\hline 954 & NSF & ENG & Civil \& mechanical systems (CMS) & Architeclural \& Mechanical Systems & $\overline{9410894}$ & & $E / G$ \\
\hline$\overline{955}$ & NSF & ENG & Civil \& mechanical systems (CMS) & Siting \& Geolechnical Syslems & 9410895 & & $E / G$ \\
\hline 956 & NSF & ENG & Civil \& mechanical systems (CMS) & Siting \& Geolechnical Syslems & 9411018 & & $E / G$ \\
\hline 957 & NSF & ENG & Civil \& mechanical systems (CMS) & Architeclural \& Mechanical Systems & 9411528 & & $E / G$ \\
\hline 958 & NSF & ENG & Civil \& mechanical systems (CMS) & Natural \& Man-Made Hazard Mitigation & 9411529 & & $E / G$ \\
\hline 959 & NSF & ENG & Bioengineering 8 environmental systems (B) & Environmental Engineering Systems & 9411572 & & $E / G$ \\
\hline 960 & $\overline{N S F}$ & $E N G$ & Electrical \& communications systems (ECS & Computational Engineering & 9411589 & & $E / G$ \\
\hline 961 & NSF & ENG & Civil \& mechanical systems (CMS) & Structural Systems & 9411738 & & $E / G$ \\
\hline 962 & NSF & CSE & Information, robotics \& intelligent systems () & Robotics \& Machine Intelligence & 9411750 & & $E / G$ \\
\hline 963 & NSF & ENG & Civil \& mechanical systems (CMS) & Architeclural \& Mechanical Systems & 9411759 & & $E / G$ \\
\hline 964 & NSF & ENG & Civil \& mechanical systems (CMS) & Structural Systems \& Construction Processes & 9412049 & & $E / G$ \\
\hline 965 & NSF & ENG & Civif \& mechanical systems (CMS) & Siting \& Geolechnical Systems & 9412759 & & $E / G$ \\
\hline 966 & NSF & ENG & Civil \& mechanical systems (CMS) & Natural \& Man-Made Hazard Mitigation & 9412905 & & $E / G$ \\
\hline 967 & NSF & ENG & Civil \& mechanical systems (CMS) & Architectural \& Mechanical Systems & 9412906 & & $E / G$ \\
\hline 968 & NSF & ENG & Civil \& mechanical systems (CMS) & Struciural Systems \& Construction Processes & 9412942 & & $E / G$ \\
\hline 969 & NSF & ENG & Civil \& mechanical systems (CMS) & Siting \& Geotechnical Systems & 9413277 & & $E / G$ \\
\hline 970 & $\bar{N} \bar{S} F$ & ENG & Civil \& mechanical systems (CMS) & Solid Mechanics & 9413372 & & $E / G$ \\
\hline 971 & NSF & ENG & Chemical \& transport systems (CTS) & Combustion \& Thermal Plasmas & 9413549 & & $E / G$ \\
\hline 972 & NSF & ENG & Civil \& mechanical systems (CMS) & Large Structural \& Building Systems & 9413725 & & $E / G$ \\
\hline 973 & NSF & ENG & Civil \& mechanical systems (CMS) & Large Structural \& Building Systems & 9413747 & & $E / G$ \\
\hline$\overline{974}$ & INSF & ENG & Civil \& mechanical systems (CMS) & Large Structural \& Building Systems & 9413859 & & $E / G$ \\
\hline 975 & NSF & ENG & Bioengineering \& environmental systems (B & Environmental Engineering Systems & 9413942 & & $E / G$ \\
\hline
\end{tabular}




\begin{tabular}{|c|c|c|c|c|c|c|c|c|c|c|c|c|c|}
\hline & Agency & $\begin{array}{l}\text { Start } \\
\text { Date }\end{array}$ & $\begin{array}{l}\text { End } \\
\text { Date } \\
\end{array}$ & \begin{tabular}{|c|} 
Place of \\
Performance \\
\end{tabular} & 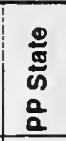 & Performer Name & \begin{tabular}{|c|c} 
Perf \\
Type
\end{tabular} & $\begin{array}{c}\text { Total } \\
\text { Award } \\
\text { Amount }\end{array}$ & $\begin{array}{c}\text { Average } \\
\text { Annual } \\
\text { Fundling }\end{array}$ & $\begin{array}{c}\text { FY } \\
\text { Total } \\
\text { Amt } \\
\end{array}$ & $\begin{array}{c}\text { FY Fed } \\
\text { Amt }\end{array}$ & $\begin{array}{c}\text { FY } \\
\text { Non- } \\
\text { Fod } \\
\text { Amt } \\
\end{array}$ & $\begin{array}{c}\text { Prod. } \\
*\end{array}$ \\
\hline 911 & NSF & Mar-94 & Dec-94 & Alameda & CA & HELLER \& CO. & IndBus & 64.352 & 64.352 & 64.352 & 64.352 & 0 & 7 \\
\hline 912 & NSF & Sep-94 & Aug-96 & Washington & $D C$ & CATHOLIC UNIVERSITY & PriEdlnst & 94.274 & 28.746 & 28.746 & 28.746 & 0 & 7 \\
\hline 913 & NSF & Dec-93 & Now-94 & New York & NY & AMER SOC OF CIVIL ENG & NonprofNonE & 21.542 & 20.654 & 20.654 & 20.654 & 0 & 7 \\
\hline 914 & NSF & Sep-94 & Nov-96 & Rictumond & CA & \begin{tabular}{|l|} 
CUREE \\
\end{tabular} & NonproNonE & 85 & 85 & 85 & 85 & 0 & 7 \\
\hline 915 & SNF & Apr-94 & Sep-95 & Reno & NV & U OF NEVADA RENO & Edlnst & 27.938 & 27.938 & 27.938 & 27.938 & 0 & 7 \\
\hline 916 & NSF & Sep-94 & Aug-96 & Southfield & MI & LAWRENCE TECHNICAL U & PriEdlnst & 136.05 & 40.7 & 40.7 & 40.7 & 0 & 7 \\
\hline 917 & INSF & Aug-94 & Jul-96 & Knoxville & $T N$ & U OF TENNESSEE KNOXV & Edinst & 49.938 & 49.938 & 49.938 & 49.938 & 0 & 8 \\
\hline 918 & NSF & Apr-94 & Oct-94 & Palo Alto & CA & STANFORD UNIVERSITY & PriEdlnst & 10 & 10 & 10 & 10 & O) & 7 \\
\hline 919 & NSF & Sep-94 & Aug-96 & Laramie & WY & UNIVERSITY OF WYOMINC & Edlnst & 218.363 & 94.638 & 94.638 & 94.638 & o) & 7 \\
\hline 920 & NSF & Oct-94 & Jul-96 & Buffalo & NY & SUNY BUFFALO & Edlnst & 330 & 211 & 211 & 211 & 0 & 7 \\
\hline 921 & INSF & Sep-94 & Jut-97 & Urbana & IL & U OF ILL URBANA-CHAMP, & Edlnst & 246.161 & 11.042 & 11.042 & 11.042 & O & 7 \\
\hline 922 & NSF & Sep-94 & Aug-97 & Hamiton & $\overline{N Y}$ & COLGATE UNIVERSITY & PriEdlnst & 83.862 & 83.862 & 83.862 & 83.862 & 0 & 7 \\
\hline 923 & NSF & Oct-94 & Aug-95 & Cincinnati & $\mathrm{OH}$ & U OF CINCINNATI & Edlnst & 30 & 30 & 30 & 30 & o) & 5 \\
\hline 924 & INSF & Oct-94 & Aug-97 & Reno & NV & U OF NEVADA RENO & Edlnst & 167.981 & 167.981 & 167.98 & 167.98 & o & 7 \\
\hline 925 & SNSF & Oct-94 & Aug-96 & Allanta & GA & GA TECH RES CORP - GIT & Edlnst & 120 & 120 & 120 & 120 & 0 & 7 \\
\hline 926 & NSF & Jun-94 & Nov-97 & Amherst (censu & $M A$ & U OF MASSACHUSETTS A & Edlnst & 149.582 & 149.582 & 149.58 & 149.58 & o| & 7 \\
\hline 927 & NSF & Oct-94 & Sep-96 & Newark & NJ & NEW JERSEY INST OF TE & Oth & 251.905 & 81 & 81 & 81 & o) & 7 \\
\hline 928 & NSF & Oct-94 & Aug-97 & Knoxville & $T N$ & U OF TENNESSEE KNOXV & Edlnst & 88.829 & 88.829 & 88.829 & 88.829 & 0 & 7 \\
\hline 929 & NSF & Sep-94 & Aug-99 & Gainesville & $\mathrm{FL}^{-}$ & U OF FLORIDA & Edlnst & 12600 & 2000 & 2000 & 2000 & 0 & 7 \\
\hline 930 & NSF & Jur-94 & Nov-95 & Mayaguez & PR & U OF PUERTO RICO MAYA & Edlnst & 49.553 & 49.553 & 49.553 & 49.553 & o. & 7 \\
\hline 931 & INSF & May-94 & Oct-95 & Raleigh & NC & NORTH CAROLINA STATE & Edlnst & 26.3 & 26.3 & 26.3 & 26.3 & of & 7 \\
\hline 932 & NSF & Apr-94 & Sep-95 & State College & PA & PA ST UUNIVERSITY PAR & Edlnst & 52.746 & 52.746 & 52.746 & 52.746 & 0 & 7 \\
\hline 933 & NSF & Mas-94 & Feb-95 & Honotuiu & $H$ & U OF HAWAII MANOA & Edlnst & 12.148 & 12.148 & 12.148 & \begin{tabular}{|l|}
12.148 \\
\end{tabular} & 0 & 7 \\
\hline 934 & NSF & Aug-94 & Jun-96 & Hous & TX & U OF HOUSTON & Edlnst & 60 & 60 & 60 & 60 & 0 & 7 \\
\hline 935 & NSF & oct-94 & Aug-95 & Lafayette & IN & PURDUE UNIV RESEARCH & Edlnst & 159.999 & 79.999 & 79.999 & 79.999 & 0 & 7 \\
\hline 936 & NSF & Sep-94 & Aug-95 & Ann Arbor & MI & UNIVERSITY OF MICHIGAN & Edlnst & 52.303 & 52.303 & 52.303 & 52.303 & 0 & 7 \\
\hline 937 & NSF & Feb-94 & Jan-95 & Buffalo & NY & SUNY BUFFALO & Edlnst & 21.8 & 21.8 & 21.8 & 21.8 & 0 & 7 \\
\hline 938 & NSF & May-94 & Sep-96 & Oakland & CÁ & EARTHQUAKE ENG RES II & Nonproñone & 94.999 & 94.999 & 94.999 & 94.999 & 0 & 7 \\
\hline 939 & NSF & Jul-94 & Jun-96 & Evanston & IL & NORTHWESTERN UNIVEA & PriEdlnst & 152 & 50 & 50 & 50 & 0 & 7 \\
\hline 940 & NSF & Aug-94 & Jun-96 & Boca Raton & FL & FLORIDA ATLANTIC UNIV & Edinst & 19.81 & 19.81 & 19.81 & 19.81 & o & 7 \\
\hline 941 & NSF & Mar-94 & Aug-95 & Worcester & $\overline{M A}$ & WORCESTER POLYTECH & PriEdlnst & 5.039 & 5.039 & 5.039 & 5.039 & 0 & 7 \\
\hline 942 & NSF & Jut-94 & May-96 & thaca & NY & CORNELL UNIVERSITY-EN & PriEdinst & 40.001 & 40.001 & 40.001 & 40.001 & 0 & 7 \\
\hline 943 & NSF & Jan-94 & Dec-94 & Berkeley & CA & U OF CAL BERKELEY & Edinst & 10 & 10 & 10 & 10 & 0 & 7 \\
\hline 944 & NSF & Aug-94 & Jut-97 & Honolulu & $\mid \mathrm{HI}$ & U OF HAWAII MANOA & Edinst & 94.959 & 94.959 & 94.959 & 94.959 & 0 & 7 \\
\hline 945 & NSF & May-94 & Sep-97 & Philadelphia & PA & DREXEL UNIVERSITY & PriEdlnst & 2000 & 2000 & 2000 & 2000 & 0 & 3 \\
\hline 948 & NSF & Oct-94 & Jut-97 & Davis & $\mathrm{CA}$ & U OF CAL DAVIS & Edinst & 100 & 100 & 100 & 100 & 0 & 7 \\
\hline 947 & NSF & Sep-94 & Jul-96 & Minneapolis & $\mathrm{MN}$ & U OF MINNESOTA-TWINC & EdInst & 93.5 & 34.27 & 34.27 & 34.27 & 0 & 7 \\
\hline 948 & NSF & Aug-94 & Jul-96 & Newark & $D E$ & UNIVERSITY OF DELAWAF & Edinst & 89.059 & 29.567 & 29.567 & 29.567 & 0 & 7 \\
\hline 949 & NSF & May-94 & Apr-95 & Boulder & $\mathrm{CO}$ & ATKINSON-NOLAND ASSQ & indBus & 3 & 3 & 3 & 3 & 0 & 7 \\
\hline 950 & NSF & Aug-94 & Dec-95 & Tampa & $\mathrm{FL}$ & UOF SOUTH FLORIDA & Edinst & 18 & 18 & 10 & 18 & o & 7 \\
\hline 951 & NSF & Sep-94 & Aug-97 & Minneapolis & $M N$ & U OF MINNESOTA-TWIN C & Edinst & 100 & 100 & 100 & 100 & $\frac{\pi}{0}$ & 7 \\
\hline 952 & NSF & Sep-94 & Jut-96 & Norman & OK & U OF OKLAHOMA & Edinst & 87.94 & 38.834 & 38.834 & \begin{tabular}{|l|}
38.834 \\
\end{tabular} & 0 & 7 \\
\hline 953 & NSF & Sep-94 & Jul-96 & Boukder - & $\mathrm{CO}$ & U OF COLORADOBOULDE & Edlnst & 90 & 30 & 30 & 30 & 0 & 8 \\
\hline 954 & NSF & May-94 & Jan-95 & EIS & CA & SOCIATES & indBus & 50 & 50 & $50^{\circ}$ & 50 & 0 & 6 \\
\hline 955 & NSF & Jun-94 & Oct-95 & Orange & $\mathrm{CA}$ & GEO-ENVIRONMENTAL IN & IndBus & 8.87 & 8.87 & 8.87 & 8.87 & 0 & 7 \\
\hline 956 & NSF & May-94 & Apr-95 & Cleveland & $\mathrm{OH}$ & CASE WESTERN RESERV & PriEdlnst & 9.6 & 9.6 & 9.6 & 9.6 & 0 & 7 \\
\hline 957 & NSF & Sep-94 & Aug-95 & Rolla & MO & U OFMISSOURI ROLLA & Edlnst & 35 & 35 & 35 & 35 & o. & 7 \\
\hline 958 & NSF & Apr-94 & Mar-95 & Fort Collins & $\mathrm{CO}$ & COLORADO STATE UNIVE & Edlnst & 1 & 1 & 1 & 1 & 0 & 7 \\
\hline 959 & NSF & Aug-94 & Jul-95 & Knoxville & $T N$ & UOF TENNESSEE KNOXV & Edlnst & 45.841 & 45.841 & 45.841 & 45.841 & 0 & 7 \\
\hline 960 & NSF & May-94 & Sep-97 & Notre Dame & WN & UNIVERSITY OF NOTRE D & PriEdlnst & 129.914 & 47.804 & 47.804 & 47.804 & 0 & 7 \\
\hline 961 & NSF & Sep-94 & May-95 & Urbana & IL & U OF ILL URBANA-CHAMP & Edinst & 134.467 & 134.467 & 134.47 & 134.47 & 0 & 7 \\
\hline 962 & NSF & Jun-94 & May-95 & Columbus & $\mathrm{OH}$ & OHIO STATE UNIV RES FO & Edlnst & 50 & 50 & 50 & 50 & 0 & 7 \\
\hline 963 & NSF & May-94 & Od-95 & Urbana & IL & U OF ILL URBANA-CHAMP & Edlnst & 39.93 & 39.93 & 39.93 & 39.93 & 0 & 7 \\
\hline 964 & NSF & Sep-94 & Jul-95 & Lafayette & IN & PURDUE UNIV RESEARCH & Edlnst & 28.75 & 28.75 & 28.75 & 28.75 & o & 5 \\
\hline 965 & NSF & Jun-94! & May-95 & Los Angeles & $\overline{C A}$ & U OF SOUTHERN CALIFOA & PriEdlnst & 35.835 & 35.835 & 35.835 & 35.835 & 0 & 7 \\
\hline 966 & NSF & Sun-94 & Od-97 & Blacksburg & VA & VA POLYTECHNIC INST \& & Edlnst & 158.862 & 35.944 & 35.944 & 35.944 & 0 & 7 \\
\hline 967 & NSF & Sep-94 & Jul-97 & Madison & WI & U OF WISCONSIN MADISC & Edinst & 180 & 180 & 180 & 180 & 0 & $\overline{7}$ \\
\hline 968 & NSF & Oct-94 & Aug-96 & Rapid City & SD & SD SCH OF MINES AND TE & Edlnst & 197.037 & 63.474 & 63.474 & 63.474 & 0 & 7 \\
\hline 969 & NSF & Jun-94 & Nov-95 & Pullman & WA & WASHINGTON STATE UNI & Edlnst & 4.79 & 4.79 & 4.79 & 4.79 & 0 & 7 \\
\hline 970 & NSF & $\operatorname{Jan} \cdot \overline{95}$ & Dec-97 & Raleigh & NC & NÖRTH CAROLINA STATE & Eälnst & 1450 & $14 \overline{50}$ & 1450 & 1450 & 0 & 7 \\
\hline 971 & NSF & Aug-94 & Jul-95 & Worcester & MA & WORCESTER POLYTECH & PriEdlnst & 101.22 & 101.22 & 101.22 & 101.22 & 0 & 7 \\
\hline 972 & NSF & Sep-94 & Aug-97 & Newark & $\mathrm{NJ}$ & \begin{tabular}{|l|l|} 
NUIT \\
\end{tabular} & Edlnst & 448.715 & 448.715 & 448.72 & 448.72 & 0 & 7 \\
\hline 973 & NSF & Sep-94 & Feb-96 & Houghton & MI & MICH TECHNOLOGICAL UI & Edlnst & 206.674 & 206.674 & 206.67 & 206.67 & 0 & 7 \\
\hline 974 & NSF & Oct-94 & Sep-96 & Irvine & $\mathrm{CA}$ & U OF CAL IRVINE & Edlnst & 230.068 & 230.068 & 230.07 & 230.07 & 0 & 7 \\
\hline 975 & NSF & $\operatorname{sep}-94$ & Aug-95 & Washington & $D C$ & CATHOLIC UNIVERSITY & PriEdlnst & 131.167 & 131.167 & 131.17 & 131.17 & 0 & 7 \\
\hline
\end{tabular}




\begin{tabular}{|c|c|c|c|c|c|c|}
\hline & Agency & Bureau & Program & Project & Award Number & $\begin{array}{l}\text { Award } \\
\text { Type }\end{array}$ \\
\hline 976 & NSF & ENG & Bioengineering \& environmental systems (E) & Environmental Engineening Systems & 9413970 & $E / G$ \\
\hline 977 & NSF & ENG & Civil \& mechanical systems (CMS) & Siting \& Geotechnical Systems & 9414375 & $E / G$ \\
\hline 978 & NSF & $\mathrm{BIO}$ & Molecular \& cellular biosciences (MCB) & Microbial Genetics & 9415295 & $E / G$ \\
\hline 979 & NSF & ENG & Civil \& mechanical systems (CMS) & Civil \& mechanical systems - Other & 9415726 & $E / G$ \\
\hline 980 & NSF & ENG & Civil \& mechanical systems (CMS) & Earthquake Systems Inlegration & 9415728 & $E / G$ \\
\hline 981 & NSF & ENG & Civil \& mochanical systems (CMS) & Civil \& mechanical systems - Other & 9415738 & $E / G$ \\
\hline 982 & NSF & ENG & Civil \& mechanical systems (CMS) & Civil \& mechanical systems - Other & 9415955 & $E / G$ \\
\hline 983 & NSF & ENG & Civil \& mechanical systems (CMS) & Civil \& mochanical systems - Other & 9416112 & E/G \\
\hline 984 & NSF & ENG & Civil \& mechanical systems (CMS) & Civil \& mechanical systems - Other & 9416151 & EJG \\
\hline 985 & NSF & ENG & Civil \& mechanical systems (CMS) & Civil \& mechanical systems - Other & 9416160 & $E / G$ \\
\hline 986 & NSF & ENG & Civil \& mochanical systems (CMS) & Civil \& mechanical systems - Other & 9416171 & E/G \\
\hline 987 & NSF & ENG & Civil \& mechanical sysiems (CMS) & Civil \& mochanical systems - Other & 9416174 & $E / G$ \\
\hline 988 & NSF & ENG & Civil \& mechanical systems (CMS) & Civil \& mechanical systems - Other & 9416175 & $E / G$ \\
\hline 989 & NSF & ENG & Civil \& mochanical systems (CMS) & Civil \& mochanical systems - Other & 9416181 & $E / G$ \\
\hline 990 & NSF & ENG & Civil \& mechanical systems (CMS) & Civil \& mechanical systems - Other & 9416183 & E/G \\
\hline 991 & NSF & ENG & Civil \& mechanical systems (CMS) & Civil \& mochanical systems - Other & 9416184 & $E / G$ \\
\hline 992 & NSF & ENG & Civil \& mechanical systems (CMS) & Civil \& mechanical systems - Other & 9416232 & $E / G$ \\
\hline 993 & NSF & ENG & Civil \& mechanical systems (CMS) & Civil \& mechanical systems - Other & 9416248 & $E / G$ \\
\hline$\overline{994}$ & NSF & ENG & Civil \& mechanical systems (CMS) & Civil \& mechanical systems - Other & 9416262 & $E / G$ \\
\hline 995 & NSF & ENG & Civil \& mechanical systems (CMS) & Civil \& mochanical systems - Other & 9416265 & E/G \\
\hline 996 & NSF & ENG & Civil \& mechanical systems (CMS) & Civil \& mechanical systems - Other & 9416266 & $E / G$ \\
\hline 997 & NSF & ENG & Civil \& mechanical systems (CMS) & Civil \& mechanical systems - Other & 9416273 & $E / G$ \\
\hline 998 & NSF & ENG & Civil \& mechanical systems (CMS) & Civil \& mechanical systems - Other & 9416274 & $E / G$ \\
\hline 999 & NSF & ENG & Civil \& mechanical systems (CMS) & Civil \& mechanical systems - Other & 9416276 & $E / G$ \\
\hline 1000 & NSF & ENG & Civil \& mechanical systems (CMS) & Civil \& mechanical systems - Other & 9416287 & $E / G$ \\
\hline 1001 & NSF & ENG & Civil \& mochanical systems (CMS) & Civil \& machanical systems - Other & 9416354 & $E / G$ \\
\hline 1002 & NSF & ENG & Civil \& mechanical systems (CMS) & Civil \& mechanical systems - Other & 9416363 & $E / G$ \\
\hline 1003 & NSF & ENG & Civil \& mechanical systems (CMS) & Civil \& mechanical systems - Other & 9416366 & $E / G$ \\
\hline 1004 & NSF & ENG & Civil \& mechanical systems (CMS) & Civil \& mechanical systems - Other & 9416378 & $E / G$ \\
\hline 1005 & NSF & ENG & Civil \& mechanical systems (CMS) & Civil \& mochanical systems - Other & 9416380 & $E / G$ \\
\hline 1006 & NSF & ENG & Civil \& mechanical systems (CMS) & Civil \& mechanical systems - Other & 9416383 & E/G \\
\hline 1007 & NSF & ENG & Civil \& mechanical systems (CMS) & Civil \& mechanical systems - Other & 9416393 & $E / G$ \\
\hline 1008 & NSF & ENG & Civil \& mechanical systems (CMS) & Civil \& mechanical systems - Other & 9416420 & $E / G$ \\
\hline 1009 & NSF & ENG & Civil \& mechanical systems (CMS) & Civil \& mochanieal systems - Other & 9416424 & $E / G$ \\
\hline 1010 & NSF & ENG & Civil \& mechanical systems (CMS) & Civil \& mechanical systems - Other & 9416441 & $E / G$ \\
\hline 1011 & NSF & ENG & Civil \& mechanical systems (CMS) & Civil \& mechanical systems - Other & 9416449 & $E / G$ \\
\hline 1012 & NSF & ENG & Civil \& mechanical systems (CMS) & Civil \& mechanical systems - Other & 9416451 & $E / G$ \\
\hline 1013 & NSF & ENG & Civil \& mechanical systems (CMS) & Civil \& mechanical systems - Other & 9416457 & $E / G$ \\
\hline 1014 & NSF & ENG & Civil \& mechanical systems (CMS) & Earthquake Systems Integration & 9416458 & $E / G$ \\
\hline 1015 & NSF & ENG & Civil \& mechanical systems (CMS) & Civil \& mechanical systems - Other & 9416463 & $E / G$ \\
\hline 1016 & NSF & ENG & Civil \& mechanical sysiems (CMS) & Civil \& mechanical systems - Other & 9416466 & $E / G$ \\
\hline 1017 & NSF & ENG & Civil \& mochanical systems (CMS) & Civil \& mechanical systems - Other & 9416469 & $E / G$ \\
\hline 1018 & NSF & ENG & Civil \& mechanical systems (CMS) & Civil \& mechanical systems - Other & 9416480 & $E / G$ \\
\hline 1019 & NSF & ENG & Civil \& mochanical systems (CMS) & Civil \& mochanical systems - Other & 9416482 & $E / G$ \\
\hline 1020 & NSF & ENG & Civil \& mechanical systems (CMS) & Civil \& mechanical syslems - Other & 9416487 & $E / G$ \\
\hline 1021 & NSF & ENG & Civil \& mechanical systems (CMS) & Civil \& mechanical systems - Other & 9416499 & $E / G$ \\
\hline 1022 & NSF & ENG & Civil \& mechanical systems (CMS) & Civil \& mochanical systems - Other & 9416509 & $E / G$ \\
\hline 1023 & NSF & ENG & Civil \& mechanical systems (CMS) & Civil \& mechanical systems - Other & 9416510 & $E / G$ \\
\hline 1024 & NSF & ENG & Civil \& mechanical systems (CMS) & Civil \& mechanical syslems - Other & 9416516 & $E / G$ \\
\hline 1025 & NSF & ENG & Civil \& mechanical systems (CMS) & Civil \& mochanical systerms - Other & 9416524 & $E / G$ \\
\hline 1026 & NSF & ENG & Civil \& mechanical systems (CMS) & Civil \& mechanical systems - Other & 9416525 & $E / G$ \\
\hline 1027 & NSF & ENG & Civil \& mechanical systems (CMS) & Civil \& mechanical systems - Other & 9416530 & $E / G$ \\
\hline 1028 & NSF & ENG & Civil \& mechanical systems (CMS) & Civil \& mochanical systems - Other & 9416533 & $E / G$ \\
\hline 1029 & NSF & ENG & Civil \& mechanical systems (CMS) & Civil \& mechanical systems - Other & 9416759 & $E / G$ \\
\hline 1030 & NSF & ENG & Civil \& mechanical systems (CMS) & Civil \& mechanical systems - Other & 9416771 & $E / G$ \\
\hline 1031 & NSF & ENG & Engineering education \& conters (EEC) & Engineering Education & 9420522 & $E / G$ \\
\hline 1032 & NSF & ENG & Engineering education \& centers (EEC) & Engineering Education & 9420583 & $E / G$ \\
\hline 1033 & NSF & ENG & Civil \& mechanical systems (CMS) & Architectural \& Mechanical Systems & 9421593 & $E / G$ \\
\hline 1034 & NSF & ENG & Electrical \& communications systems (EC) & Computational Engineering & 9422730 & $E / G$ \\
\hline 1035 & NSF & EHR & Graduate education \& research developme & nl (GER) & 9454022 & $E / G$ \\
\hline 1036 & NSF & ENG & Civil \& mechanical systems (CMS) & Architectural \& Mechanical Systems & 9457288 & $E / G$ \\
\hline 1037 & NSF & ENG & Civil \& mechanical systems (CMS) & Large Structural \& Building Systems & 9457305 & $E / G$ \\
\hline 1038 & NSF & MPS & Chemistry (CHE) & Statistical \& Simulations & 9457365 & $E / G$ \\
\hline 1039 & NSF & ENG & Civil \& mechanical systems (CMS) & Large Structural \& Building Systems & 9457604 & $E / G$ \\
\hline 1040 & NSF & ENG & Civil \& mechanical systems (CMS) & Structural Systems & 9496181 & $E / G$ \\
\hline
\end{tabular}




\begin{tabular}{|c|c|c|c|c|c|c|c|c|c|c|c|c|c|}
\hline & Ageney & $\begin{array}{l}\text { Start } \\
\text { Date }\end{array}$ & $\begin{array}{l}\text { End } \\
\text { Date }\end{array}$ & $\begin{array}{c}\text { Place of } \\
\text { Performance }\end{array}$ & \begin{tabular}{l}
$:$ \\
\multirow{3}{3}{} \\
0 \\
0 \\
0
\end{tabular} & Performer Name & \begin{tabular}{|c|c|c} 
& \\
Perf & \multicolumn{5}{|c}{} \\
Type & 0 \\
\end{tabular} & $\begin{array}{l}\text { Total } \\
\text { Award } \\
\text { Amount }\end{array}$ & $\begin{array}{c}\text { Average } \\
\text { Annual } \\
\text { Funding }\end{array}$ & $\begin{array}{c}\text { FY } \\
\text { Total } \\
\text { Amt }\end{array}$ & $\begin{array}{c}\text { FY Fed } \\
\text { Amt }\end{array}$ & $\begin{array}{l}\text { Fon } \\
\text { Non- } \\
\text { Fed } \\
\end{array}$ & $\begin{array}{c}\text { Prod. } \\
\#\end{array}$ \\
\hline 976 & NSF & Sep-94 & Aug-97 & Grand Forks & IND & U OF NORTH DAKOTA & Edinst & 191.983 & 191.983 & 191.98 & 191.98 & 0 & 7 \\
\hline 977 & NSF & Jun-94 & May-95 & Berkeley & $C A$ & OF CAL BERKELEY & Edlnst & 20 & 20 & 20 & 20 & 0 & 7. \\
\hline 978 & NSF & Jul-94 & Jun-96 & Alhens & $G A$ & UOF GEORGIA RES FDNI & Edlnst & 49.9841 & 49.984 & 49.984 & 49.984 & 0 & 7 \\
\hline 979 & NSF & Sep-94 & Jul-95 & Uibana & IL & UOFILI URBANA-CHAMP & Edlnst & 601 & 60 & 60 & 60 & of & 7 \\
\hline 980 & NSF & Aug-94 & Jul-95 & Eas Lansing & $\mathrm{MI}$ & MICHIGAN STATE UNIVER & Edinst & 99.638 & 99.638 & 99.538 & 99.638 & 0 & \\
\hline 981 & NSF & Aug-94 & JuL-96 & Nowark & $\mathrm{DE}$ & UNIVERSITY OF DELAWA & Edinst & 124.881 & 92.562 & 92.562 & 92.562 & $0 !$ & \\
\hline 982 & NSF & Sep-94 & Aug-95 & Reno & NV & UOF NEVADA RENO & Edlnst & 46.788 & 46.788 & 46.788 & 46.788 & & \\
\hline 983 & NSF & Sep-94 & Aug-95 & Seattle & WA & UOF WASHINGTON & Edlnst & 65 & 65 & -65 & 65 & 0 & 7 \\
\hline 984 & NSF & Sep-94 & Aug-95 & Cleveland & $\mathrm{OH}$ & CASE WESTERN RESERV & PriEdlnst & 49.046 & 49.046 & 49.046 & 49.046 & 이 & 7 \\
\hline 985 & NSF & Sep-94 & Aug-95 & Invine & $C A$ & UOF CAL IRVINE & Edlnst & 40 & 40 & 40 & 40 & 0 & 7 \\
\hline 986 & NSF & Sep-94 & Aug-95 & Blacksburg & VA & VA POLYTECHNIC INST \& & Edlnst & 65 & 65 & 65 & 65 & 0 & 7 \\
\hline 987 & $\overline{N S F}$ & Sep-94] & Aug-95 & Pasadena & CA & CALIFORNIA INST OF TEC & PriEdinst & 35 & 35 & 35 & 35 & 0 & 7 \\
\hline 988 & NSF & Sep-94 & Aug-95 & Lutherville & $M D$ & DYNAMIC IN SITU GEOTEC & IndBus & 78 & 52 & 52 & 52 & 0 & 7 \\
\hline 989 & NSF & Aug 94 & Jul-95 & Oakland & $C A$ & ASSN OF BAY AREA GOV & StalecGor & -65 & -65 & -65 & 65 & 0 & 7 \\
\hline 990 & NSF & Sop-94 & $J \mid(t)-95$ & San Francisco & CA & BUILDING SYSTEMS DEVI & IndBus & $90.161 !$ & 90.161 & $90.16: 1$ & 90.161 & 0 & \\
\hline 9911 & NSF- & Sep-94 & Jui-95 & Anin Arbo: & Iin & UNIVERSITY OFMICHIGAP & Edlnst & 69.945 & 59.945 & $69 . \overline{945}$ & 69.945 & 0 & \\
\hline 992 & NSF & Sep-94i & Aug-95 & San Francisco & $C A$ & EQE INTERNATIONAL INC & indBus & 50 & $50 !$ & 50 & 50 & 0 & 7 \\
\hline 995i & NSF & Sep-94 & 4ug-9E. & Troy & NY & RENYSESLAER POLYTECIT: & PriEdins: & की: & 5uj & 60 & $-50 !$ & & \\
\hline $994 ;$ & ANSF & Sep-sit & Alig-95: & Knoxvite & Tn & ¿USF IENNESSEE KNOXU & Esilrsi & 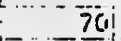 & $7 i$ ! & 30 & 70 & & \\
\hline 995 & NSF & Sep-94 & Aug-95 & Borkeley & $\mathrm{CA}^{-}$ & U OF CAL BERKELEY & Eánst & $-\overline{80}$ & 80 & 80 & $\theta \bar{C}$ & 0 & \\
\hline 996 & NSF & Aug-94 & Jul-95 & Bethlehem & $P A$ & LEHIGH UNIVERSITY & PriEdlnst & 100.067 & 100.067 & 100.07 & 100.07 & 0 & \\
\hline 997 & NSF & 0ct-94 & Sep-95 & Los Angetes & $C A$ & UOF SOUTHERN CALIFOA & PriEdlnst & 74.205 & 74.205 & 74.205 & 74.205 & 0 & \\
\hline 998 & NSF & Sep-94: & Jul-95 & Bellilet & $\overline{P A}$ & LEHIGH UNIVERSITY & PriEdlnst & $52.137 \mathrm{i}$ & 52.137 & 52.137 & 52.137 & 0 & \\
\hline 999 & NSF & Sep-94 & Auto. & San Diego & $C A$ & UOF CAL SAN DIEGO & Edinst & $60 \mathrm{i}$ & 60 & sol & 60 & v & \\
\hline 1000 & NSF & Sep-94 & Aug-95 & Austin & TX & UOF TEXAS AUSTIN & Edinst & $70^{\dagger}$ & 70 & 701 & 70 & & \\
\hline 1001 & NNSF & Sep-94! & Aug-95 & Urbana & IIL & UOF ILL URBANA-CHAMP & Edlnst & 60 & 60 & 60 & 60 & 0 & 7 \\
\hline 1002 & NSF & Sep-94: & Aug-95 & Minneapolis & $\overline{M N}$ & A-TWIN C & Edlnst & 65 & 65 & 65 & 65 & & \\
\hline $10 \overline{0}$ & NSF & Sep-94 & Aug 95 & Los Ang & $C A$ & DAMES \& MOORE LOS AN & indBus & 60 & 60 & 60 & 60 & 0 & \\
\hline 1004 & NSF & Oct-94 & Aug-95 & Provo & UT & M YOUNG UNIVEA & PriEdinst & 60 & $-\overline{60}+$ & -60 & 60 & $0_{1}$ & \\
\hline 1005 & NSF & $\operatorname{sep}-\frac{1}{2}$ & Aug-95! & Northridge (Nor & $1 C A$ & UNIV CORP NORTHRIDGE & Edlinst & 65.116 & 65.116 & 65.116 & 65.116 & & 7 \\
\hline 1006 & NSF & Sep-94! & Aug-95 & $\operatorname{LOS} A$ & $\overline{C A}$ & UOF CAL LOS ANGELES & Esinst & 60 & 60 & 60 & 60 & 0 & 6 \\
\hline 1007 & NSE & sep-94] & A $j 095$ & San Francisco & Cä & H JDEGENKOLB ASSOCS & indeus & 11.942 & 11.942 & 11.942 & 11.942 & 0 & 7 \\
\hline 1008 & NSF & Aug-94 & Jul-95 & PabAlio & $C_{A}$ & VERSITY & PriEdlnst & 82.159 & 82.159 & 82.159 & 82.159 & 0 & 7 \\
\hline $1009_{\mathrm{i}}$ & iNSF & Oct-94 & Sep-95 & Sacramento & CA & $D O C$ & StalocGor & 52.515 & 52.515 & 52.515 & 52.515 & 0 & \\
\hline 1010 & INSE & Aug-94 & Jul-95 & Berkeley & $C A$ & UOF CAL BERKELEY & Edlnst & 57.248 & 57.248 & 57.248 & 57.248 & & 7 \\
\hline 1011 & NSF & Sep-94 & Aug-95 & Northbrook & IIL & WISS JANNEY ELSTNER\&: & IndBus & 15 & 15 & 15 & 15 & o: & \\
\hline 1012 & NSF & Oct-94 & Sep-95i & Heights & $C A$ & WCC & IndBus & 65.366 & 65.366 & 65.366 & 65.366 & & 7 \\
\hline 1013 & NSF & Sep-94 & Ju!-95 & Berkeley & $C A$ & UOF CAL BERKELEY & Edlnst & 100 & 100 & 100 & 100 & & \\
\hline 1014 & NSF & Sep-94 & Aug-95 & Allania & GA & GA TECH RES CORP - GIT & Edlnst & 87.464 & 87.464 & 87.464 & 87.464 & & 7 \\
\hline 1015 & NSF & Sep-94 & Aug-95 & Pretie & MO & UOF MISSOURI ROLLLA & Edlnst & 83.346 & 83.346 & $83.346 i$ & 83.346 & & \\
\hline 1016 & NSS & Sep-94 & Aug -95i & Seatte & WA & UOFW & Edlnst & 54.999 & 54.999 & 54.999 & 54.999 & & \\
\hline 1017 & NSEF & Sep-94 & ALg-95 & iniediora & $\overline{M A}$ & IUTIS UNIVERSITY & Micenst & 50 & 60 & 50 & 60 & 8 & 7 \\
\hline $10 \div 8$ & $\mathrm{NSF}^{-}$ & Sep-94! & Allg-95: & Buffalo & 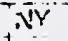 & SUINY BUTFALO & Edingt & & $90^{+}$ & 80 & & & \\
\hline 1019 & NSF & Sep-94 & Jul-95 & Berke & CA & UOFC & Edlnst & 49.058 & 49.058 & 49.058 & 49.058 & & \\
\hline 1020 & NSF & Sep -94 & Aug-S5 & Potsdam & $N Y$ & CLARKSON UNIVERSIT & PriÉlinsi & 60 & 60 & -60 & 60 & & \\
\hline 1021 & INSF & Aug-94 & Jul-95 & Urbana & $\pi$ & CHAMP: & Edlnst & 79.257 & 79.257 & 79.257 & 79.257 & & 6 \\
\hline 1022 & $\overline{N S F}$ & Sep-94! & Aug -95 & Los Angeles & $\overline{C A}$ & CALIFOF & PriEdlnst & 50.155 & 50.155 & 50.155 & 50.155 & & \\
\hline 1023 & NSF & Oct-94 & Aug -95 & Berke & $\mathrm{CA}$ & $S O C \operatorname{ING}$ & indBis & 50.468 & 50.468 & 50.468 & 50.468 & & \\
\hline 1024 & NSF & Aug-94 & Jul-95 & Berkeley & $C A$ & UOF CAL BERKELEY & Edlnst & 70 & 70 & 70 & 70 & & \\
\hline 1025 & $N S F$ & Sep-94 & Augg-95 & San Luis Obisp. & XCA & NIVERSTH & Edinst & 57.997 & 57.997 & 57.997 & 57.997 & & \\
\hline 1026 & NSF & Sep-94 & Aug -95 & Austin & $T X$ & UOF TEXAS AUSTIN & Edinst & 70.746 & 70.746 & 70.746 & 70.746 & 0 & 7. \\
\hline 1027 & NSF & Sep-94 & & & CA & SEISMIC ISO & IndBus & 46.278 & 46.278 & 46.278 & 46.278 & & \\
\hline 1028 & NSF & Sep-94 & Aug-95 & San Francisco & $C A$ & RUTHEF & IndBus & 17.315 & 17.315 & 17.315 & 17.315 & & 7 \\
\hline 1029 & NSF & Sep-94 & & & $\operatorname{tin}$ & PURDU: & Edlnst & 80 & 80 & 80 & 80 & 0 & \\
\hline 1030 & NSF & Sep-94 & Aug-95 & Los Angeles & CA & UOF SOUTH & PriEdlnst & 60 & 60 & 60 & 60 & & 7 \\
\hline 1031 & NSF & OCt-94 & & Atlanta & GA & GA TECH RES & Edlnst & 300 & 220.018 & 220.02 & 220.02 & & \\
\hline 1032 & NSF & oct-94 & Aug-96 & Baltimore & $M D$ & JOHNS HOPKINS UNIVER & PriEdlnst & $310.5 !$ & 98.436 & 98.436 & 98.436 & & 7 \\
\hline 1033 & NSF. & act-94 & & Austin - & TX & U OF TEXAS AUSTIN & Edlnst & 82.062 & 52.5 & 52.5 & 52.5 & ot & \\
\hline 103 & NSF & Oct-94 & Sep-97: & Urbàna & il & NA-CHAMP & Edlnst & 1268.4 & 422.801 & 422.8 & 422.8 & & \\
\hline $10 \overline{3}$ & NSF & Sep-94 & Aug- 98 & College & MD & UOF MD COL & Edlnst & 562.5 & 112.5 & $11 z .5$ & 112.5 & & \\
\hline 1036 & NSF & Sep-94 & Jul-98 & Ames & AA & IOWA STATE & Edlnst & 275 & 25 & 25 & 25 & of & 7 \\
\hline 1037 & $\overline{N S F}$ & Aug-94! & Jun- -98 & New York & $\bar{N} \overline{N Y}$ & COLUMBIAU & PriEdinst & 275 & & 25 & 25 & & \\
\hline 1038 & NSF & AuF 94 & Jun-98: & Davis. & $\overline{C A}$ & UOF CAL DAVIS & Edlns & 312.5 & 62.5 & 62.5 & 62.5 & 0 & \\
\hline 1039 & INSF & AUS-94! & Ju!-98 & Las Crices & NMM & SEN MEXIEO ST UN!YERS & ẼIns & 275 & 25 & 25 & 25 & 0 & 7 \\
\hline 1040 & NSF & Apr -94 & Sep-94 & San Diegr & $\mathrm{CA}$ & IJ OF CAL SAN DIESO & Edinst & 13.532 & 47.846 & 47.846 & 47.846 & & \\
\hline
\end{tabular}


C\&B R\&D Repon

\begin{tabular}{|c|c|c|c|c|c|c|}
\hline & Agency & Bureau & Program & Project & Award Number & $\begin{array}{l}\text { Award } \\
\text { Type }\end{array}$ \\
\hline 1042 & NSF & ENG & Civil \& mechanical systems (CMS) & Large Structural \& Building Systems & 9496285 & $E / G$ \\
\hline
\end{tabular}


C\&B R\&D Report

\begin{tabular}{|c|c|c|c|c|c|c|c|c|c|c|c|c|c|c|}
\hline & Agency & $\begin{array}{l}\text { Start } \\
\text { Date }\end{array}$ & $\begin{array}{l}\text { End } \\
\text { Date }\end{array}$ & $\begin{array}{c}\text { Place of } \\
\text { Performance }\end{array}$ & $\begin{array}{l}\Phi \\
\stackrel{\Phi}{\Xi} \\
\tilde{a} \\
a\end{array}$ & Performer Name & $\begin{array}{c}\text { Perf } \\
\text { Type }\end{array}$ & 동 & $\begin{array}{c}\text { Total } \\
\text { Award } \\
\text { Amount }\end{array}$ & $\begin{array}{l}\text { Average } \\
\text { Annual } \\
\text { Funding }\end{array}$ & $\begin{array}{l}\text { FY } \\
\text { Total } \\
\text { Amt }\end{array}$ & $\begin{array}{c}\text { FY Fod } \\
\text { Amt }\end{array}$ & $\begin{array}{c}\text { FY } \\
\text { Non- } \\
\text { Fed } \\
\text { Amt }\end{array}$ & $\begin{array}{c}\text { Prod. } \\
\text { : }\end{array}$ \\
\hline 1041 & NSF & Sep-94 & Feb-95 & Lafayetle & IN & PURDUE UNIV RESEARCH & Edlnst & & 12.7 & 92.8 & 92.8 & 92.8 & 0 & 7 \\
\hline 1042 & NSF & Sep-94 & Apr-96 & Pullman & WA & WASHINGTON STATE UNI & Edlnst & & 54.2 & & & & & 7 \\
\hline
\end{tabular}




\section{Appendix D: \\ Sample Award Abstracts}

Two pages of sample Award Abstracts have been included in this section to provide the reader with a sense of the kind of information available in RaDiUS. Because the complete printout of Award Abstracts is several hundred pages long, this abbreviated version has been supplied. The actual Abstracts range in length from one line to ten pages, but the majority are similar to those listed below. Readers interested in obtaining project abstracts for any particular project should consult the contacts listed in Appendix B for availability information. 
AWARD :: LONG DESCR: Base Isolation of structures and systems is an attractive strategy to mitigate the effects of earthquakes.

Implementation of the strategy, on a limited basis, has been achieved in several countries world- wide. Many issues, which require considerable research, remain unresolved and are barriers to further accelerated implementation. This research project address many of these issues in both conventional structures and for bridges. This includes soilstructure instruction effects, materials behavior under seismic conditions and actual performance of structures on a shaker table simulator. The research will provide design and analysis methodologies to aid in accelerated implementation of base isolation. This research grant provides the base support to Dr. Constantinou for a Presidential Young Investigator Award. ::

8857749

8858549

\section{SHORT DESCR: PRESIDENTIAL YOUNG INVESTIGATORS} AWARD :: LONG DESCR: The research will involve the analysis and design of high accuracy mechanical systems. Such systems include machines to perform finishing tasks on construction sites such as: 1) Wallbots, robots to assemble steel track and stud walls; and 2) Scaffbot, robot to perform maintenance and assembly tasks on vertical exterior surfaces of ships and building. Also included are machines to advance the state of the art of precision surveying and navigation, such as: 1) Acall, a machine to calibrate accelerometers, for the National Bureau of Standards, to achieve accuracies of one part per million. This type of accuracy is needed to allow slow moving vehicles to use inertial systems to navigate for extended periods of time. 2) Molecular Measuring Machine, a machine being designed with the National Bureau of Standards with the capability to measure the position of atoms on specimens centimeters in diameter.::

SHORT DESCR: PRESIDENTIAL YOUNG INVESTIGATOR AWARD :: LONG DESCR: Under this Presidential Young Investigator Award (PYI), the following studies will be pursued: (1) Dynamic response of linear and nonlinear secondary systems, (2) Reliability of systems with uncertain parameters, (3) Identification and optimization of complex systems, and (4) Vibration reduction of dynamic systems These studies are aiming at improving the understanding of the dynamic behaviorof complex structures with subsystem components and the seismic design of such structures. This project is initiated in FY 88 and will run for five years. :: 

PRACTICES IN THE 21ST CENTURY TO BE HELD AT WORCESTER POLYTECHNIC INSTITUTE WORCESTER MA. :: LONG DESCR: This award is to support a $21 / 2$ day conference on fire engineering and design considerations. The issues to be discussed are barriers to institutional, political, and economic with respect to the transfer of recent research results to designers and regulatory bodies. Proceedings will be published. ::

SHORT DESCR: JOINT U.S.-ITALY-YUGOSLAVIA RESEARCH ON EVALUATION AND RETROFIT OF MASONRY-INFILLED REINFORCED CONCRETE FRAMES :: LONG DESCR: This ia a joint project between the University of Colorado and the consulting engineering firm of Atkinson-Noland and Associates, Inc. of Boulder, Colorado, in cooperation with engineers from Italy and Yugoslavia. The project will investigate the lateral load resistance of masonry-infilled reinforced concrete structures to determine the feasibility of using the method to reinforce existing reinforced concrete buildings and to evaluate the safety of existing buildings. ::

SHORT DESCR: CONTROL AND PERFORMANCE OF CENTRALIZED HEATING AND COOLING SYSTEMS :: LONG DESCR: The objective of the proposed research is to develop techniques that would allow a building equipped with an energy management system to be operated in a cost efficient manner. These techniques would be implemented in the energy management system and automatically utilize operating data to perform a number of important functions including on-line optimal control, performance monitoring and fault detection. Methodologies for optimal control strategies would be developed to allow the plant to operate at minimum cost. These methodologies would involve development of simulation models for the plant and the components, with parameter estimation techniques employed that utilize operating data. Fault detection would allow assessment of changes in plant performance and identification of the sources of the change. Both analytical and experimental approaches will be employed to test the validity of the methodologies. The results of the research will be algorithms that can be directly incorporated in energy management systems. :: 


